\title{
AN ALGORITHMIC VERSION OF THE HYPERGRAPH REGULARITY METHOD
}

\author{
P.E. HAXELL, B. NAGLE, AND V. RÖDL
}

\begin{abstract}
Extending the Szemerédi Regularity Lemma for graphs, P. Frankl and V. Rödl [14] established a 3-graph Regularity Lemma guaranteeing that all large triple systems $\mathcal{G}_{n}$ admit bounded partitions of their edge sets, most classes of which consist of regularly distributed triples. Many applications of this lemma require a companion Counting Lemma [30], allowing one to find and enumerate subhypergraphs of a given isomorphism type in a "dense and regular" environment created by the 3-graph Regularity Lemma. Combined applications of these lemmas are known as the 3-graph Regularity Method. In this paper, we provide an algorithmic version of the 3-graph Regularity Lemma which, as we show, is compatible with a Counting Lemma. We also discuss some applications.
\end{abstract}

\section{INTRODUCTION}

Szemerédi's Regularity Lemma [45] is one of the most important tools in combinatorics, with applications ranging across combinatorial number theory, extremal graph theory and theoretical computer science (see $[28,29]$ for excellent surveys of applications). This lemma hinges on the notion of $\varepsilon$-regularity. A bipartite graph $H=(X \cup Y, E)$ is $(d, \varepsilon)$-regular if for every $X^{\prime} \subseteq X$, $\left|X^{\prime}\right|>\varepsilon|X|$, and $Y^{\prime} \subseteq Y,\left|Y^{\prime}\right|>\varepsilon|Y|$, we have $\left|d_{H}\left(X^{\prime}, Y^{\prime}\right)-d\right|<\varepsilon$ where $d_{H}\left(X^{\prime}, Y^{\prime}\right)=$ $\left|H\left[X^{\prime}, Y^{\prime}\right]\right| /\left(\left|X^{\prime}\right|\left|Y^{\prime}\right|\right)$ is the density of the bipartite graph $H\left[X^{\prime}, Y^{\prime}\right]$ induced on the sets $X^{\prime}$ and $Y^{\prime}$. (In this paper, graphs and hypergraphs are identified with their edge sets.) We say $H$ is $\varepsilon$-regular if it is $(d, \varepsilon)$-regular for some $d$. Szemerédi's Regularity Lemma is stated as follows.

Theorem 1.1 (Szemerédi's Regularity Lemma [45]). For all $\varepsilon>0$ and integers $t_{0} \geq 1$, there exist integers $T_{0}=T_{0}\left(\varepsilon, t_{0}\right)$ and $N_{0}=N_{0}\left(t_{0}, \varepsilon\right)$ so that every graph $G$ on $N>N_{0}$ vertices admits a partition of its vertex set $V(G)=V_{1} \cup \cdots \cup V_{t}, t_{0} \leq t \leq T_{0}$, satisfying

(1) $V(G)=V_{1} \cup \cdots \cup V_{t}$ is equitable: $\left|V_{1}\right| \leq \cdots \leq\left|V_{t}\right| \leq\left|V_{1}\right|+1$;

(2) $V(G)=V_{1} \cup \cdots \cup V_{t}$ is $\varepsilon$-regular: all but $\varepsilon\left(\begin{array}{l}t \\ 2\end{array}\right)$ pairs $V_{i}, V_{j}, 1 \leq i<j \leq t$, are $\varepsilon$-regular.

Much of the strength of Szemerédi's Regularity Lemma rests on the ability to embed fixed subgraphs within appropriate parts of an $\varepsilon$-regular partition, a phenomenon formally due to the following easily proved graph 'Counting Lemma'.

Fact 1.2 (Counting Lemma). For all integers $k$ and non-negative d, there exists $\varepsilon_{0}>0$ so that for all $0<\varepsilon<\varepsilon_{0}$, whenever $H=\bigcup_{1 \leq i<j \leq k} H^{i j}$ is a $k$-partite graph on $V_{1} \cup \ldots \cup V_{k}$, $\left|V_{1}\right|=\ldots=\left|V_{k}\right|=n$, where each $H^{i j}, 1 \leq i<j \leq k$, is $(d, \varepsilon)$-regular, then the number of $k$-cliques in $H,\left|\mathcal{K}_{k}^{(2)}(H)\right|$, satisfies $d^{\left(\begin{array}{c}k \\ 2\end{array}\right)} n^{k}\left(1-\varepsilon^{1 / k}\right) \leq\left|\mathcal{K}_{k}^{(2)}(H)\right| \leq d^{\left(\begin{array}{c}k \\ 2\end{array}\right) n^{k}}\left(1+\varepsilon^{1 / k}\right)$.

Combined applications of Theorem 1.1 and Fact 1.2 are known as the Graph Regularity Method and will be discussed shortly.

The first author was partially supported by NSERC.

The second author was partially supported by NSF Grant DMS 0639839.

The third author was partially supported by NSF Grant DMS 0300529. 
The great importance of Szemerédi's Regularity Lemma has led to a search for extensions to $k$-uniform hypergraphs, for example $[6,10,15,16,17]$. While these early generalizations did lead to some interesting applications, they did not seem to capture the full power of Szemerédi's lemma for graphs. In particular, they did not allow for the embedding of small subsystems within a regular structure. The first hypergraph regularity lemma shown to admit a corresponding counting lemma was due to Frankl and Rödl [14] for 3-uniform hypergraphs (we shall refer to 3-uniform hypergraphs as 3-graphs, for short). In what follows, we refer to this Regularity Lemma for 3-uniform hypergraphs as the 3R-Lemma, for short. The 3R-Lemma guarantees that any large 3 -graph $\mathcal{G}$ admits a bounded partition of its triples, most classes of which are "regularly distributed". The corresponding 3-graph Counting Lemma was due to Frankl, Nagle and Rödl [14, 30]. Joint applications of these lemmas are known as the 3-graph Regularity Method which has been used in several hypergraph problems [9, 21, 23, 24, 31, 37, 43].

The original proof of Szemerédi's Regularity Lemma for graphs was not algorithmic. An algorithmic version of Szemerédi's Lemma was later established in [1, 2] by Alon, Duke, Lefmann, Rödl and Yuster (see also [13]), rendering constructive solutions to many problems where Szemerédi's Lemma is applied (see [1] for applications). Correspondingly,

\section{the object of this paper is to establish compatible algorithmic} versions of the 3R-Lemma and the Counting Lemma.

Results in this paper were announced in our earlier paper [11] (see the Abstract of [11]), and outlined in the extended abstract [20]. We state our results precisely in Section 2.

Extending the 3R-Lemma, regularity lemmas and counting lemmas for $k$-uniform hypergraphs, also allowing the embedding of small substructures, were recently developed by Gowers [18, 19] and Nagle, Rödl, Schacht and Skokan [32, 40]. Most likely, it would be possible to extend our current work to provide an algorithmic version of the general $k$-graph regularity method. It appears that our approach here has some similarities with that of Gowers [18]. In particular, we use the concept of a hypergraph having 'minimally many' copies of $K_{2,2,2}^{(3)}$, where $K_{2,2,2}^{(3)}$ is the complete 3 -partite 3 -graph with two vertices in each class (we call this concept ' $(\alpha, \delta)$-minimal'). In [18], Gowers uses a related concept (cf. ' $\alpha$-quasirandom'), although the arguments in our paper are quite different from those in [18].

To accomplish the object of this paper, one seeks to use the approach of Alon et al. $[1,13]$ for the graph case. Extending their approach in this paper becomes technical for several reasons. For one, the 3R-Lemma and Counting Lemma of [14,30] involve more technical environments. More importantly, however, and as we discuss momentarily, the approach taken in $[1,13]$ is based on the implication ' $C_{4}$-minimality implies regularity', the analogy of which fails to be true in the context of the 3R-Lemma (cf. (8) and our earlier work with Y. Dementieva [11]). To handle this problem, our paper does the following:

(1) formulates a variant of the Regularity Lemma from [14] in upcoming Theorem 2.4;

(2) modifies the approach of Alon et al. $[1,13]$ to prove Theorem 2.4 constructively;

(3) formulates and proves a counting lemma in upcoming Theorem 2.7 which is compatible with Theorem 2.4.

The work of this paper represents some departure from the original 3R-Lemma of [14] and the original Counting Lemma from [30]. To motivate our work, Section 1.1 reviews the original approach of Alon et al. for constructively proving Szemerédi's Regularity Lemma. Section 1.2 then emphasizes which parts from Section 1.1 shall be the same in this paper and illustrates how we modify the remaining parts. In Section 2, we then precisely state the main results 
of our paper and discuss an application. The remainder of the paper proves the main results discussed in Section 2.

1.1. Constructive proof of the regularity lemma. The algorithmic version of Szemerédi's Regularity Lemma states that for each $\varepsilon>0$ and $t_{0}$, for each graph $G$ on $N>N_{0}\left(\varepsilon, t_{0}\right)$ vertices, an $\varepsilon$-regular $t$-equitable partition $V(G)=V_{1} \cup \cdots \cup V_{t}, 1 \leq t \leq T_{0}=T_{0}\left(\varepsilon, t_{0}\right)$, (the existence of which is guaranteed by Theorem 1.1) can be constructed in time polynomial in $N$. In the immediate sequel, we sketch the constructive proof of Statement (2) of Theorem 1.1 (the essence of Theorem 1.1) where it is enough to consider the special case $t_{0}=1$.

The proof boils down to one central problem. Indeed, let $\varepsilon>0$ be fixed and let bipartite graph $H$ be given with bipartition $X \cup Y$ and positive density $d=|H| /(|X||Y|)>0$. As we explain below, to prove Theorem 1.1 constructively, one is faced with the problem of

efficiently deciding if $H$ is $\varepsilon$-regular,

a problem which, unfortunately, is co-NP-complete [1]. To handle (1), one circumvents the problem by counting the number $\left|\mathcal{K}_{2,2}^{(2)}(H)\right|$ (cf. (42)) of copies of $K_{2,2}^{(2)}=C_{4}$ appearing in $H$, an operation easily completed in time $|X|^{2}|Y|^{2}$. It is always the case that $\left|\mathcal{K}_{2,2}^{(2)}(H)\right| \geq d^{4}\left(\begin{array}{c}|X| \\ 2\end{array}\right)\left(\begin{array}{c}|Y| \\ 2\end{array}\right)(1-$ $o(1))$ where $o(1) \rightarrow 0$ as $\min \{|X|,|Y|\} \rightarrow \infty$. We now consider the relationship between $\varepsilon$ regularity and the number $\left|\mathcal{K}_{2,2}^{(2)}(H)\right|$.

On the one hand, it is not difficult to show that

$$
H \text { is } \varepsilon \text {-regular } \Longrightarrow\left|\mathcal{K}_{2,2}^{(2)}(H)\right| \leq(d+f(\varepsilon))^{4}\left(\begin{array}{c}
|X| \\
2
\end{array}\right)\left(\begin{array}{c}
|Y| \\
2
\end{array}\right)
$$

for a fixed function $f(\varepsilon)>\varepsilon$ satisfying $f(\varepsilon) \rightarrow 0$ as $\varepsilon \rightarrow 0$. Moreover, the same proof gives

(3) $\left|\mathcal{K}_{2,2}^{(2)}(H)\right|>(d+f(\varepsilon))^{4}\left(\begin{array}{c}|X| \\ 2\end{array}\right)\left(\begin{array}{c}|Y| \\ 2\end{array}\right) \quad \Longrightarrow \quad \exists O(|X||Y|)$-time algorithm which builds

$$
X^{\prime} \subset X \text { and } Y^{\prime} \subset Y,\left|X^{\prime}\right|>\varepsilon|X| \text { and }\left|Y^{\prime}\right|>\varepsilon|Y| \text {, such that }\left|d_{H}\left(X^{\prime}, Y^{\prime}\right)-d\right|>\varepsilon .
$$

(We shall often refer to sets $X^{\prime}, Y^{\prime}$ as above as witnesses of irregularity). On the other hand, convexity arguments show $[1,13]$

$$
\left|\mathcal{K}_{2,2}^{(2)}(H)\right| \leq(d+g(\varepsilon))^{4}\left(\begin{array}{c}
|X| \\
2
\end{array}\right)\left(\begin{array}{c}
|Y| \\
2
\end{array}\right) \quad \Longrightarrow \quad H \text { is } \varepsilon \text {-regular }
$$

for a fixed (positive) function $g(\varepsilon)<\varepsilon$. We note the following crucial remark.

Remark 1.3. For the fixed functions $f$ and $g$ in (2) and (4), respectively, suppose $\left|\mathcal{K}_{2,2}^{(2)}(H)\right|>$ $(d+g(\varepsilon))^{4}\left(\begin{array}{c}|X| \\ 2\end{array}\right)\left(\begin{array}{c}|Y| \\ 2\end{array}\right)$. Then from $(2), H$ is not $f^{-1}(g(\varepsilon))$-regular, where from $f(\varepsilon)>\varepsilon>g(\varepsilon)$ we necessarily have $f^{-1}(g(\varepsilon))<g(\varepsilon)<\varepsilon$. As such, $H$ may simultaneously be both $\varepsilon$-regular and $f^{-1}(g(\varepsilon))$-irregular.

We now use the implications in (2)-(4) to give the constructive proof of Theorem 1.1 from [1]. Indeed, with $\varepsilon>0$ fixed and $N$-vertex graph $G$ given, one iteratively builds a special sequence of partitions $\mathcal{P}_{\ell}: V(G)=V_{1}^{(\ell)} \cup \cdots \cup V_{t_{\ell}}^{(\ell)}, \ell \geq 1$, and defines for each $\ell \geq 1$ the corresponding index (cf. $[44,45]))$

$$
\text { ind } \mathcal{P}_{\ell}=\frac{1}{N^{2}} \sum_{1 \leq i<j \leq t_{\ell}} d_{G}^{2}\left(V_{i}^{(\ell)}, V_{j}^{(\ell)}\right)\left|V_{i}^{(\ell)}\right|\left|V_{j}^{(\ell)}\right|,
$$

noting that ind $\mathcal{P}_{\ell}$ never exceeds one. 
Suppose partitions $\mathcal{P}_{1}, \ldots, \mathcal{P}_{\ell}$ have been constructed where $\mathcal{P}_{\ell}: V(G)=V_{1}^{(\ell)} \cup \cdots \cup V_{t_{\rho}}^{(\ell)}$. We seek to know if $\mathcal{P}_{\ell}$ is $\varepsilon$-regular (for if it is, then $\mathcal{P}_{\ell}$ is the partition desired). Recalling (1), however, we can not, in general, efficiently decide the regularity status of $G\left[V_{a}^{(\ell)}, V_{b}^{(\ell)}\right], 1 \leq a<$ $b \leq t_{\ell}$, for a given $\varepsilon>0$. As such, we instead count $\left|\mathcal{K}_{2,2}^{(2)}\left(G\left[V_{a}^{(\ell)}, V_{b}^{(\ell)}\right]\right)\right|$. With $d_{a b}^{(\ell)}$ denoting the density of $G\left[V_{a}^{(\ell)}, V_{b}^{(\ell)}\right]$ and with the functions $f$ and $g$ given in (2) and (4), resp., we compute $\left|\mathcal{K}_{2,2}^{(2)}\left(G\left[V_{a}^{(\ell)}, V_{b}^{(\ell)}\right]\right)\right|$ and record the following information:

(1) if $\left|\mathcal{K}_{2,2}^{(2)}\left(G\left[V_{a}^{(\ell)}, V_{b}^{(\ell)}\right]\right)\right| \leq\left(d_{a b}^{(\ell)}+g(\varepsilon)\right)^{4}\left(\begin{array}{c}\left|V_{a}^{(\ell)}\right| \\ 2\end{array}\right)\left(\begin{array}{c}\left|V_{b}^{(\ell)}\right| \\ 2\end{array}\right)$, then $G\left[V_{a}^{(\ell)}, V_{b}^{(\ell)}\right]$ is $\varepsilon$-regular by (4);

(2) if $\left|\mathcal{K}_{2,2}^{(2)}\left(G\left[V_{a}^{(\ell)}, V_{b}^{(\ell)}\right]\right)\right|>\left(d_{a b}^{(\ell)}+g(\varepsilon)\right)^{4}\left(\begin{array}{c}\left|V_{a}^{(\ell)}\right| \\ 2\end{array}\right)\left(\begin{array}{c}\left|V_{b}^{(\ell)}\right| \\ 2\end{array}\right)$, then $G\left[V_{a}^{(\ell)}, V_{b}^{(\ell)}\right]$ is not $f^{-1}(g(\varepsilon))$ regular (cf. Remark 1.3), and by (3), we may efficiently construct witnesses $\widetilde{V}_{a}^{(\ell)}$ and $\widetilde{V}_{b}^{(\ell)}$ of the $f^{-1}(g(\varepsilon))$-irregularity of $G\left[V_{a}^{(\ell)}, V_{b}^{(\ell)}\right]$.

Correspondingly, if

$\left(1^{\prime}\right)$ all but $\varepsilon\left(\begin{array}{c}t_{\ell} \\ 2\end{array}\right)$ of the bipartite graphs $G\left[V_{a}^{(\ell)}, V_{b}^{(\ell)}\right], 1 \leq a<b \leq t_{\ell}$, have the 'minimal number' of $K_{2,2}^{(2)}$ 's, i.e., such that (4) is satisfied, then the partition $\mathcal{P}_{\ell}$ is $\varepsilon$-regular, as desired;

$\left(2^{\prime}\right)$ at least $f^{-1}(g(\varepsilon))\left(\begin{array}{c}t_{\ell} \\ 2\end{array}\right)<\varepsilon\left(\begin{array}{c}t_{\ell} \\ 2\end{array}\right)$ of the bipartite graphs $G\left[V_{a}^{(\ell)}, V_{b}^{(\ell)}\right], 1 \leq a<b \leq t_{\ell}$, don't have the minimal number of $K_{2,2}^{(2)}$ 's, then by (3), we have constructed a system of witnesses $\widetilde{V}_{a}^{(\ell)}$ and $\widetilde{V}_{b}^{(\ell)},\{a, b\} \in I^{(\ell)}$ for some 'large' indexing set $I^{(\ell)} \subseteq\left(\begin{array}{c}{\left[t_{\ell}\right]} \\ 2\end{array}\right)$, for the $f^{-1}(g(\varepsilon))$-irregularity of $G\left[V_{a}^{(\ell)}, V_{b}^{(\ell)}\right]$.

While $\left(1^{\prime}\right)$ signifies the end of the proof, $\left(2^{\prime}\right)$ puts one directly in the center of Szemerédi's proof $[44,45]$ of the Regularity Lemma.

Indeed, with system of constructed witnesses $\widetilde{V}_{a}^{(\ell)}$ and $\widetilde{V}_{b}^{(\ell)},\{a, b\} \in I^{(\ell)}$, as above, Szemerédi's original proof $[44,45]$ establishes the existence of a partition $\mathcal{P}_{\ell+1}: V(G)=V_{1}^{(\ell+1)} \cup$ $\cdots \cup V_{t_{\ell+1}}^{(\ell+1)}$ for which

$$
t_{\ell+1} \leq t_{\ell} 2^{t_{\ell}-1}
$$

and

$$
\text { ind } \mathcal{P}_{\ell+1} \geq \text { ind } \mathcal{P}_{\ell}+\frac{\left(f^{-1}(g(\varepsilon))\right)^{5}}{2} \text {. }
$$

Moreover, since the system of witnesses $\widetilde{V}_{a}^{(\ell)}$ and $\widetilde{V}_{b}^{(\ell)},\{a, b\} \in I^{(\ell)}$, was already constructed, Szemerédi's proof $[44,45]$ can be easily made to construct the partition $\mathcal{P}_{\ell+1}$ in time $O(N)$. As such, within $2 /\left(f^{-1}(g(\varepsilon))\right)^{5}$ iterations $\ell$ of the process above (recall the index never exceeds one), one must have constructed an $\varepsilon$-regular partition $\mathcal{P}^{(\ell)}$, as desired.

1.2. Modifying the graph approach. We see from the outline of the previous section that the constructive proof of Szemerédi's Regularity Lemma hinges on the relationship between the $\varepsilon$-regularity of a bipartite graph $H$ and the number of $K_{2,2}^{(2)}$, s of $H$. The implications in (2)-(4) assert that the property of $\varepsilon$-regularity in graph $H$ is essentially equivalent to the parameter $\left|\mathcal{K}_{2,2}^{(2)}(H)\right|$ being asymtotically minimized over all bipartite graphs of the same density.

The concept of 3 -graph regularity in [14] is so-called $(\delta, r)$-regularity for 3 -graphs $\mathcal{H}$. While we define this concept precisely in Definition 3.1 below, we say, for now, that it plays the role of $\varepsilon$-regularity in Szemerédi's Regularity Lemma and is, correspondingly, the central concept in 
the Counting Lemma from [14, 30]. Similarly to (1), it is a co-NP-complete problem to verify the property of $(\delta, r)$-regularity, and thus as in (2)-(4), we seek an easily verifiable property which is essentially equivalent to $(\delta, r)$-regularity. Following the outline of the previous section, a primary candidate would be to consider the number of $K_{2,2,2}^{(3)}$ 's in a 3 -graph $\mathcal{H}$, where we recall $K_{2,2,2}^{(3)}$ is the complete 3 -partite 3 -graph with two vertices in each class. In Definition 2.2 below, we define the appropriate sense in which a 3 -graph $\mathcal{H}$ contains 'minimally-many' copies of $K_{2,2,2}^{(3)}$ (viz. $(\alpha, \delta)$-minimality). Then, one hopes to transparently extend the graph approach above, going between $\left(\delta_{1}, r\right)$-regularity and $\left(\alpha, \delta_{2}\right)$-minimality analogously to how we went between $\varepsilon$-regularity and ' $K_{2,2}^{(2)}$-minimality' with (2)-(4).

The problem in extending the approach above is that, unlike the case of graphs where one has the equivalence established in (2)-(4),

$$
\left(\delta_{1}, r\right) \text {-regularity is not equivalent to }\left(\alpha, \delta_{2}\right) \text {-minimality. }
$$

In particular, our earlier work in [11] established that (with appropriately quantified constants)

$$
\left(\delta_{1}, r\right) \text {-regularity implies }\left(\alpha, \delta_{2}\right) \text {-minimality }
$$

but that

$$
\left(\alpha, \delta_{2}\right) \text {-minimality does not imply }\left(\delta_{1}, r\right) \text {-regularity. }
$$

(The details of (9) will, in this paper, be discussed in Proposition 3.2 below.) Then (10) implies that Step $\left(1^{\prime}\right)$ of the graph outline above can not be extended to the context of $\left(\delta_{1}, r\right)$-regular hypergraphs. We now very roughly indicate the main idea we take in resolving this technicality. (The precise details are given in Section 3.)

Unlike Frankl and Rödl's Regularity Lemma, we formulate our algorithmic Regularity Lemma in terms of $\left(\alpha, \delta_{2}\right)$-minimality, meaning that for a given 3-graph $\mathcal{H}$ on $n$ vertices, we want to construct a partition $\mathcal{P}$, most 'parts' of which have minimally many $K_{2,2,2}^{(3)}$ 's. Although the concepts are (technically) different, the main idea is similar to Szemerédi's proof $[44,45]$. Let $\mathcal{P}_{i}$ be a constructed partition for $\mathcal{H}$.

- If $\mathcal{P}_{i}$ already has most parts with minimally many $K_{2,2,2}^{(3)}$ 's, then our algorithm terminates with $\mathcal{P}_{i}$, as desired.

- If $\mathcal{P}_{i}$ has many parts, each with too many $K_{2,2,2}^{(3)}$ 's, then by (9), these parts can't be $\left(\delta_{1}, r\right)$-regular (and witnesses against the $\left(\delta_{1}, r\right)$-regularity can be found in time $O\left(n^{5}\right)$ ). Now, however, we are philosophically at the center of Frankl and Rödl's proof: we may refine $\mathcal{P}_{i}$ to obtain a new partition $\mathcal{P}_{i+1}$ whose index (defined later) is significantly larger than that of $\mathcal{P}_{i}$.

The rough sketch above indicates that we find a minimal partition $\mathcal{P}$ rather than a $\left(\delta_{1}, r\right)$ regular one. A main result in this paper (upcoming Theorem 2.7) asserts that

the concept of $\left(\alpha, \delta_{2}\right)$-minimality admits a corresponding Counting Lemma.

As such, we don't need to find a $\left(\delta_{1}, r\right)$-regular partition, as did Frankl and Rödl, in order to cooperatively apply a Counting Lemma.

Acknowledgement. We would like to thank an anonymous referee for a careful reading of this paper, and for suggestions which led to a much improved exposition. 


\section{Main Results of PAPeR}

In this section, we state the main results of this paper, an Algorithmic 3R-Lemma, Theorem 2.4, and a corresponding 3-graph Counting Lemma, Theorem 2.7. We conclude the section with an application of our theorems to a constructive hypergraph problem.

To state Theorems 2.4 and 2.7, we require some notation and concepts. We shall begin this section by presenting concepts needed to state Theorem 2.4.

2.1. 3R-partitions. Given a 3 -graph $\mathcal{G}$ with vertex set $V$, the 3 R-Lemma guarantees partitions of the vertices $V$ and the pairs $\left(\begin{array}{c}V \\ 2\end{array}\right)$, with respect to which $\mathcal{G}$ behaves 'regularly'. In the sequel, we refer to such families of partitions as $3 R$-partitions. We now describe the vertex and pair structure of 3R-partitions.

Let $V$ be a set with $|V|=N$ and let integers $\ell$ and $t$ and reals $\gamma>0$ and $\varepsilon>0$ be given. An $(\ell, t, \gamma, \varepsilon)$-partition $\mathcal{P}$ of $\left(\begin{array}{c}V \\ 2\end{array}\right)$ is an (auxiliary) partition $V=V_{0} \cup V_{1} \cup \cdots \cup V_{t}$ of $V$, together with a system of edge-disjoint bipartite graphs $\mathcal{B}=\left\{P_{a}^{i j}: 1 \leq i<j \leq t, 0 \leq a \leq \ell_{i j} \leq \ell\right\}$, such that

(1) $\left|V_{0}\right|<t$ and $\left|V_{1}\right|=\left|V_{2}\right|=\cdots=\left|V_{t}\right|=\left\lfloor\frac{N}{t}\right\rfloor \stackrel{\text { def }}{=} n$,

(2) $\bigcup_{a=0}^{\ell_{i j}} P_{a}^{i j}=K\left(V_{i}, V_{j}\right)$ is a partition of the complete bipartite graph $K\left(V_{i}, V_{j}\right)$ for all $1 \leq i<j \leq t$

(3) setting $\mathcal{B}_{\text {reg }}$ to be the collection of those bipartite graphs from $\mathcal{B}$ that are $\varepsilon$-regular, we have

$$
\sum_{P_{a}^{i j} \in \mathcal{B}_{\mathrm{reg}}}\left|P_{a}^{i j}\right|>(1-\gamma)\left(\begin{array}{l}
t \\
2
\end{array}\right) n^{2} .
$$

An $(\ell, t, \gamma, \varepsilon)$-partition $\mathcal{P}$ is said to be equitable if for all but $\gamma\left(\begin{array}{c}t \\ 2\end{array}\right)$ pairs $1 \leq i<j \leq t,\left|P_{0}^{i j}\right| \leq \gamma n^{2}$ and $P_{a}^{i j}$ has density $d_{P_{a}^{i j}}\left(V_{i}, V_{j}\right)$ satisfying $\left|d_{P_{a}^{i j}}\left(V_{i}, V_{j}\right)-\ell^{-1}\right|<\varepsilon$ for all $a=1, \ldots, \ell_{i j}$.

To describe the 'triple structure' of 3R-partitions requires still more definitions and notation. For a fixed set $V$, let an $(\ell, t, \gamma, \varepsilon)$-partition $\mathcal{P}$ of $V$ be given. Any 3-partite graph $P \subseteq \mathcal{B}$ of the form

$$
P=P_{a}^{i j} \cup P_{b}^{j k} \cup P_{c}^{i k}, \quad 1 \leq i<j<k \leq t, \quad 0 \leq a \leq \ell_{i j}, \quad 0 \leq b \leq \ell_{j k}, \quad 0 \leq c \leq \ell_{i k},
$$

is called a triad. Denote by $\operatorname{Triad}(\mathcal{P})$ the set of all such triads $P$. For $P \in \operatorname{Triad}(\mathcal{P})$, let $\mathcal{K}_{3}^{(2)}(P)$ denote the system of triangles of $P$ :

$$
\mathcal{K}_{3}^{(2)}(P)=\left\{\{x, y, z\} \in\left(\begin{array}{l}
V \\
3
\end{array}\right):\{x, y, z\} \text { induces a triangle } K_{3}^{(2)} \text { in } P\right\} .
$$

Now, let $\mathcal{G}$ be a 3 -graph on vertex set $V=V(\mathcal{G})$ where $V$ has $(\ell, t, \gamma, \varepsilon)$-partition $\mathcal{P}$. For $P \in \operatorname{Triad}(\mathcal{P})$, we write $\mathcal{G}_{P}=\mathcal{G} \cap \mathcal{K}_{3}^{(2)}(P)$ and define the density of $\mathcal{G}_{P}$ with respect to $P$ as $\alpha_{P}=d_{\mathcal{G}_{P}}(P)=\left|\mathcal{G}_{P}\right| /\left|\mathcal{K}_{3}^{(2)}(P)\right|$. Set

$$
\mathcal{K}_{2,2,2}^{(3)}\left(\mathcal{G}_{P}\right)=\left\{J \in\left(\begin{array}{l}
V \\
6
\end{array}\right): J \text { induces a copy of } K_{2,2,2}^{(3)} \text { in } \mathcal{G}_{P}\right\}
$$

where $K_{2,2,2}^{(3)}$ is the complete 3 -partite 3 -graph with 2 vertices in each class. 
2.2. Algorithmic regularity lemma. Our regularity lemma hinges on the concept of $(\alpha, \delta)$ minimality which is defined for the following environment.

Setup 2.1. Let $P=P^{12} \cup P^{23} \cup P^{13}$ be a 3-partite graph and $\mathcal{H}$ be a 3-partite 3-graph satisfying the following conditions:

(1) $\mathcal{H}$ and $P$ have common 3-partition $V=V(P)=V_{1} \cup V_{2} \cup V_{3},\left|V_{1}\right|=\left|V_{2}\right|=\left|V_{3}\right|=n$;

(2) $P^{i j}$ is $\left(\ell^{-1}, \varepsilon\right)$-regular for each $1 \leq i<j \leq 3$;

(3) $\mathcal{H} \subseteq \mathcal{K}_{3}^{(2)}(P)$ and $d_{\mathcal{H}}(P)=\alpha$.

We note that Setup 2.1 models the environment of a 'typical' triad $P_{a}^{i j} \cup P_{b}^{j k} \cup P_{c}^{i k}$ from an equitable $(\ell, t, \gamma, \varepsilon)$-partition $\mathcal{P}$ of $\left(\begin{array}{c}V \\ 2\end{array}\right)$ where, here, $V=V(\mathcal{G})$ is the vertex set of a 3-graph $\mathcal{G}$ and $\mathcal{H}$ plays the role of $\mathcal{G} \cap \mathcal{K}_{3}^{(2)}(P)$.

We will confirm in Section 4 that that, with $\varepsilon$ sufficiently small, Setup 2.1 ensures

$$
\left|\mathcal{K}_{2,2,2}^{(3)}(\mathcal{H})\right| \geq \frac{\alpha^{8}}{\ell^{12}}\left(\begin{array}{l}
n \\
2
\end{array}\right)^{3}\left(1-\varepsilon^{1 / 10}\right)
$$

(see upcoming Proposition 4.1). The following definition is therefore motivated.

Definition $2.2((\alpha, \delta)$-minimality). For $\delta>0$, we say 3-graph $\mathcal{H}$, as in Setup 2.1, is $(\alpha, \delta)$ minimal with respect to $P$ if

$$
\left|\mathcal{K}_{2,2,2}^{(3)}(\mathcal{G})\right| \leq \frac{\alpha^{8}}{\ell^{12}}\left(\begin{array}{l}
n \\
2
\end{array}\right)^{3}(1+\delta) .
$$

If $\mathcal{H}$ is not $(\alpha, \delta)$-minimal with respect to $P$, then we say $\mathcal{H}$ is $(\alpha, \delta)$-excessive with respect to $P$.

We now define a notion of 'minimality' for an $(\ell, t, \gamma, \varepsilon)$-partition $\mathcal{P}$ of $\mathcal{G}$. For $\alpha_{0}, \delta>0$, we first set

$$
\begin{gathered}
\operatorname{Triad}_{\left(\alpha_{0}, \delta\right)-\operatorname{exc}}(\mathcal{P})=\left\{P \in \operatorname{Triad}(\mathcal{P}): \mathcal{G}_{P}=\mathcal{G} \cap \mathcal{K}_{3}^{(2)}(P) \text { is }\left(\alpha_{P}, \delta\right) \text {-excessive w.r.t. } P\right. \\
\text { where } \left.d_{\mathcal{G}_{P}}(P)=\alpha_{P} \geq \alpha_{0}\right\} .
\end{gathered}
$$

We say partition $\mathcal{P}$ is $\left(\alpha_{0}, \delta\right)$-minimal with respect to $\mathcal{G}$ if

$$
\sum_{P \in \operatorname{Triad}_{\left(\alpha_{0}, \delta\right)-\text { exc }}(\mathcal{P})}\left|\mathcal{K}_{3}^{(2)}(P)\right|<\delta N^{3},
$$

and $\left(\alpha_{0}, \delta\right)$-excessive with respect to $\mathcal{G}$ otherwise. For future reference, we make the following remark.

Remark 2.3. As defined, every triad $P=P_{a}^{i j} \cup P_{b}^{j k} \cup P_{c}^{i k} \in \operatorname{Triad}_{\left(\alpha_{0}, \delta\right) \text {-exc }}(\mathcal{P})$ necessarily satisfies that each of $P_{a}^{i j}, P_{b}^{j k}, P_{c}^{i k}$ is $(1 / \ell, \varepsilon)$-regular. In particular, the set $\operatorname{Triad}(\mathcal{P})$ splits into three classes: those which are minimal, those which are excessive and those which contain an irregular bipartite graph.

We arrive at our first main theorem.

Theorem 2.4 (algorithmic regularity lemma). For all reals $\alpha_{0}, \delta, \gamma>0$, integers $t_{0}$ and $\ell_{0}$ and functions $\varepsilon: \mathbb{N}^{+} \rightarrow(0,1)$, there exist integers $T_{0}, L_{0}$ and $N_{0}$ so that every 3-graph $\mathcal{G}$ on $N>N_{0}$ vertices admits an equitable and $\left(\alpha_{0}, \delta\right)$-minimal $(\ell, t, \gamma, \varepsilon(\ell))$-partition $\mathcal{P}$ where $\ell_{0} \leq \ell \leq L_{0}$ and $t_{0} \leq t \leq T_{0}$. Moreover, there exists an algorithm which produces the partition $\mathcal{P}$ in time $O\left(N^{6}\right)$. 
In our proof of Theorem 2.4, the running time $O\left(N^{6}\right)$ will be easy to see. It is not, however, optimal. Indeed, combining ideas from $[26,27]$ with some from the current paper, one can prove a running time of $O\left(N^{4}\right)$. It seems likely that a running time of $O\left(N^{3}\right)$ should be possible. However, as refinements to the running time are not our focus in this paper, we do not discuss the issue here.

One can also prove the following form of Theorem 2.4 which may be slightly more convenient in some applications.

Theorem 2.5. For all $\alpha_{0}, \delta>0$, integers $t_{0}$ and $\ell_{0}$ and functions $\varepsilon: \mathbb{N}^{+} \rightarrow(0,1)$, there exist integers $T_{0}, L_{0}$ and $N_{0}$ so that for every 3-graph $\mathcal{G}$ on vertex set $V,|V|=N>N_{0}$, there exist integers $\ell_{0} \leq \ell \leq L_{0}$ and $t_{0} \leq t \leq T_{0}$ and a partition $\mathcal{P}$ of $\left(\begin{array}{c}V \\ 2\end{array}\right)$ with the following properties:

(1) $\mathcal{P}$ has auxiliary partition $V=V_{1} \cup \ldots \cup V_{t}$ split as evenly as possible, i.e., $\left|V_{1}\right| \leq \ldots \leq$ $\left|V_{t}\right| \leq\left|V_{1}\right|+1$

(2) For each $1 \leq i<j \leq t$, we have $K\left(V_{i}, V_{j}\right)=\bigcup_{1 \leq a \leq \ell} P_{a}^{i j}$ where for each $1 \leq a \leq \ell$, the graph $P_{a}^{i j}$ is $\left(\ell^{-1}, \varepsilon(\ell)\right)$-regular.

(3) For all but $\delta t^{3} \ell^{3}$ triads $P \in \operatorname{Triad}(\mathcal{P})$ of density $d_{\mathcal{G}_{P}}(P)=\alpha_{P} \geq \alpha_{0}$, we have that $P$ is $\left(\alpha_{P}, \delta\right)$-minimal with respect to $\mathcal{G}_{P}$.

Moreover, there exists an algorithm which produces partition $\mathcal{P}$ in time $O\left(N^{6}\right)$.

While we only sketch the details, Theorem 2.5 follows from Theorem 2.4 by employing ideas considered in [32]. In particular, one can, in time $O\left(N^{2}\right)$, alter a partition $\mathcal{P}_{2.4}$ produced by Theorem 2.4 to obtain a partition $\mathcal{P}_{2.5}$ promised by Theorem 2.5. Indeed, first equitably distribute vertices of the 'garbage class' $V_{0}$ of $\mathcal{P}_{2.4}$ into $V_{1} \cup \cdots \cup V_{t}$. It is easy to show that this process interferes with the regularity/minimality of $\mathcal{P}_{2.4}$ by at most a measure of $o(1) \rightarrow 0$ as $N \rightarrow \infty$. Now, fix $1 \leq i<j \leq t$, where we suppose there are $r_{i j} \leq \ell_{i j} \leq \ell$ many $\left(\ell^{-1}, \varepsilon(\ell)\right)$-regular bipartite graphs $P_{a}^{i j}, 1 \leq a \leq \ell_{i j}$. It is not hard to show that the union of these $r_{i j}$ graphs is itself $\left(r_{i j} / \ell, r_{i j} \varepsilon(\ell)\right)$-regular, and therefore, $\left(r_{i j} / \ell, \ell \varepsilon(\ell)\right)$-regular. It then easily follows that the complement of this union (which contains $P_{0}^{i j}$ and the $\ell_{i j}-r_{i j}$ many $\left(\ell^{-1}, \varepsilon(\ell)\right)$-irregular bipartite graphs $\left.P_{a}^{i j}\right)$ is $\left(1-r_{i j} / \ell, \ell \varepsilon(\ell)\right)$-regular. As such, one 'randomly' slices this complement into $\ell-r_{i j}$ new bipartite graphs $R_{a}^{i j}$, each of which is (almost surely) $\left(\ell^{-1}, 3 \ell \varepsilon(\ell)\right)$-regular (see Lemma 30, p. 129, of [32]). Moreover, in time $O\left(N^{2}\right)$, this random slicing may be algorithmically derandomized using Lemma 3.8, p. 144, of [22]. Repeating this procedure for all $1 \leq i<j \leq t$ produces the partition $\mathcal{P}_{2.5}$ promised by Theorem 2.5. (Note that triads $P$ which were originally $\left(\alpha_{P}, \delta\right)$-minimal w.r.t. $\mathcal{G}_{P}$ are unaffected by the process above since each bipartite graph of $P$ was already $\left(\ell^{-1}, \varepsilon(\ell)\right.$ )-regular (cf. Remark 2.3).)

2.3. Counting Lemma. We prove a hypergraph Counting Lemma compatible with our algorithmic regularity lemma, Theorem 2.4. In what follows, for a hypergraph $\mathcal{G}$ on vertex set $V$ and an integer $k$, let $\mathcal{K}_{k}^{(3)}(\mathcal{G})$ denote the system of $k$-cliques in $\mathcal{G}$ :

$$
\mathcal{K}_{k}^{(3)}(\mathcal{G})=\left\{K \in\left(\begin{array}{l}
V \\
k
\end{array}\right): K \text { induces a clique } K_{k}^{(3)} \text { of size } k \text { in } \mathcal{G}\right\} .
$$

Our Counting Lemma takes place in the following environment.

Setup 2.6. Suppose

(1) $P=\bigcup_{1 \leq i<j \leq k} P^{i j}$ is a $k$-partite graph and $\mathcal{H}=\bigcup_{1 \leq h<i<j \leq k} \mathcal{H}^{\text {hij }} \subseteq \mathcal{K}_{3}^{(2)}(P)$ is a $k$ partite 3-graph, each with $k$-partition $V(P)=V(\mathcal{H})=V_{1} \cup \ldots \cup V_{k},\left|V_{1}\right|=\ldots=\left|V_{k}\right|=$ $n$. 
(2) Each graph $P^{i j}, 1 \leq i<j \leq k$, is $(1 / \ell, \varepsilon)$-regular.

(3) Each 3-graph $\mathcal{H}^{h i j} \subseteq \mathcal{K}_{3}^{(2)}\left(P^{h i} \cup P^{i j} \cup P^{h j}\right), 1 \leq h<i<j \leq k$, is $(\alpha, \delta)$-minimal with respect to $P^{h i} \cup P^{i j} \cup P^{h j}$, i.e., $d_{\mathcal{H}^{h i j}}\left(P^{h i} \cup P^{i j} \cup P^{h j}\right)=\alpha$ and $\left|\mathcal{K}_{2,2,2}^{(3)}\left(\mathcal{H}^{h i j}\right)\right| \leq$ $\frac{\alpha^{8}}{\ell^{12}}\left(\begin{array}{l}n \\ 2\end{array}\right)^{3}(1+\delta)$.

In the environment of Setup 2.6 and with appropriately chosen constants, we estimate $\left|\mathcal{K}_{k}^{(3)}(\mathcal{H})\right|$ with the following Counting Lemma.

Theorem 2.7 (Counting Lemma). For all integers $k$ and $\alpha>0$ there exists $\delta_{0}>0$ so that for all $0<\delta<\delta_{0}$ and integers $\ell$ there exists $\varepsilon>0$ so that, with $n$ sufficiently large, whenever $\mathcal{H}=\bigcup_{1 \leq h<i<j \leq k} \mathcal{H}^{\text {hij }}$ and $P=\bigcup_{1 \leq i<j \leq k} P^{i j}$ are as in Setup 2.6 with these constants, then

$$
\left|\mathcal{K}_{k}^{(3)}(\mathcal{H})\right|=\frac{\alpha^{\left(\begin{array}{c}
k \\
3
\end{array}\right)}}{\ell^{\left(\begin{array}{c}
k \\
2
\end{array}\right)}} n^{k}\left(1 \pm \delta^{\frac{1}{120 k}}\right) .
$$

We mention that our error term is not optimal, and is taken in this paper for convenience.

2.4. Application: constructive fractional packings. In [1, 13], and more recently [4], a number of applications are given using the algorithmic version of Szemerédi's Regularity Lemma. It is likely that the work of the current paper allows some of these applications to be extended to a 3-uniform hypergraph setting. In what follows, however, we discuss an application of our work to a different problem, which concerns constructive fractional packings.

Let fixed 3-graph $\mathcal{F}$ be given. For a 3 -graph $\mathcal{G}$, let $(\underset{\mathcal{F}}{\mathcal{G}})$ denote the set of copies $\mathcal{F}_{0}$ of $\mathcal{F}$ contained in $\mathcal{G}$. An $\mathcal{F}$-packing of $\mathcal{G}$ is a collection of pairwise edge-disjoint elements of $(\underset{\mathcal{F}}{\mathcal{G}})$. We denote by $\nu_{\mathcal{F}}(\mathcal{G})$ the maximum size of an $\mathcal{F}$-packing of $\mathcal{G}$. A fractional $\mathcal{F}$-packing of $\mathcal{G}$ is any function $\psi:(\underset{\mathcal{F}}{\mathcal{G}}) \rightarrow[0,1]$ such that for every fixed edge $e \in \mathcal{G}$, we have $\sum_{(\underset{\mathcal{F}}{\mathcal{G}}) \ni \mathcal{F}_{0} \ni e} \psi\left(\mathcal{F}_{0}\right) \leq 1$. Then, $\nu_{\mathcal{F}}^{*}(\mathcal{G})$ is defined to be the maximum value of $\sum_{\mathcal{F}_{0} \in\left(\frac{\mathcal{G}}{\mathcal{F}}\right)} \psi\left(\mathcal{F}_{0}\right)$ taken over all fractional $\mathcal{F}$ packings of $\mathcal{G}$. It is clear that $\nu_{\mathcal{F}}^{*}(\mathcal{G}) \geq \nu_{\mathcal{F}}(\mathcal{G})$ holds for all 3-graphs $\mathcal{G}$.

While computing $\nu_{\mathcal{F}}(\mathcal{G})$ is an NP-hard problem (cf. [12]), computing $\nu_{\mathcal{F}}^{*}(\mathcal{G})$ is known to be a linear programming problem (and hence can be done in polynomial time). Extending a result of the first and third author [22] for graphs, the current authors proved in [21] that

$$
\nu_{\mathcal{F}}^{*}(\mathcal{G})-\nu_{\mathcal{F}}(\mathcal{G})=o\left(|V(\mathcal{G})|^{3}\right)
$$

holds for all 3-graphs $\mathcal{G}$.

Theorems 2.4 and 2.7 may be combined to give the following constructive extension of (16).

Theorem 2.8. For all fixed 3-graphs $\mathcal{F}$ and constants $\varepsilon>0$, there exists $N_{0}$ so that for all 3-graphs $\mathcal{G}$ on $N>N_{0}$ vertices, an $\mathcal{F}$-packing of $\mathcal{G}$ of size $\nu_{\mathcal{F}}(\mathcal{G})-\varepsilon N^{3}$ can be constructed in polynomial time.

Proving Theorem 2.8 requires attention to a few technical details. We defer its proof to a forthcoming paper.

2.5. Organization of paper. The remainder of this paper is organized as follows. In Section 3, we prove Theorem 2.4, the Regularity Lemma. In Section 4, we present some facts on $(\alpha, \delta)$ minimality we need to prove Theorem 2.7, the Counting Lemma. In Section 5, we prove Theorem 2.7. In Section 7, we show how upcoming Proposition 3.2 follows from our earlier work, Lemma 5.8 of [11]. 


\section{Proof of Theorem 2.4}

Our proof of Theorem 2.4 follows the outline set forth in Sections 1.1 and 1.2 of the Introduction, but uses the hypergraph language established in this paper as well as in Frankl and Rödl [14]. We begin by reviewing some concepts from [14], beginning with the crucial concept of $(\delta, r)$-regularity (compare this with the concept of our paper, $(\alpha, \delta)$-minimality - Definition 2.2).

Definition 3.1. Let $\delta>0$ and integer $r$ be given. We say 3-graph $\mathcal{H}$ is $(\delta, r)$-regular with respect to graph $P$ if for any sequence $\mathbf{Q}_{r}=\left(Q_{1}, \ldots, Q_{r}\right)$ of subgraphs $Q_{s} \subseteq P, 1 \leq s \leq r$,

$$
\left|\bigcup_{s=1}^{r} \mathcal{K}_{3}^{(2)}\left(Q_{s}\right)\right|>\delta\left|\mathcal{K}_{3}^{(2)}(P)\right| \Longrightarrow\left|d_{\mathcal{H}}\left(\mathbf{Q}_{r}\right)-d_{\mathcal{H}}(P)\right|<\delta
$$

where

$$
d_{\mathcal{H}}\left(\mathbf{Q}_{r}\right)=\frac{\left|\mathcal{H} \cap \bigcup_{s=1}^{r} \mathcal{K}_{3}^{(2)}\left(Q_{s}\right)\right|}{\left|\bigcup_{s=1}^{r} \mathcal{K}_{3}^{(2)}\left(Q_{s}\right)\right|}
$$

is the density of $\mathbf{Q}_{r}$ with respect to $\mathcal{H}$. If $\mathcal{H}$ is not $(\delta, r)$-regular with respect to $P$, we say $\mathcal{H}$ is $(\delta, r)$-irregular with respect to $P$, and in this case, any vector $\mathbf{Q}_{r}=\left(Q_{1}, \ldots, Q_{r}\right)$ violating the regularity condition above is said to be a witness of the $(\delta, r)$-irregularity of $\mathcal{H}$ with respect to $P$.

(In the definition above, when $\bigcup_{s=1}^{r} \mathcal{K}_{3}^{(2)}\left(Q_{s}\right)=\emptyset$, we shall define $d_{\mathcal{H}}\left(\mathbf{Q}_{r}\right)=\emptyset$.)

The paper [14] also extends the notion of the graph 'index' (cf. (5)) to hypergraphs. For a 3 -graph $\mathcal{G}$ with $(\ell, t, \gamma, \varepsilon)$-partition $\mathcal{P}$, define the index of $\mathcal{P}$ with respect to $\mathcal{G}$ as

$$
\text { ind } \mathcal{P}=\frac{1}{N^{3}} \sum_{P \in \operatorname{Triad}(\mathcal{P})} d_{\mathcal{G}_{P}}^{2}(P)\left|\mathcal{K}_{3}^{(2)}(P)\right| .
$$

Similarly to (5), it is easy to see that ind $\mathcal{P} \leq 1$.

We need one final preparation before proceeding to the proof of Theorem 2.4. As in (14) where we defined the family $\operatorname{Triad}_{\left(\alpha_{0}, \delta\right) \text {-exc }}\left(\mathcal{P}_{s}\right)$ of all 'excessive' triads, we define

$$
\operatorname{Triad}_{(\delta, r) \text {-irr }}(\mathcal{P})=\left\{P \in \operatorname{Triad}(\mathcal{P}): \mathcal{G}_{P}=\mathcal{G} \cap \mathcal{K}_{3}^{(2)}(P) \text { is }(\delta, r) \text {-irregular w.r.t. } P\right\}
$$

as the family of all 'irregular' triads. (By our definitions, a triad $P \in \operatorname{Triad}_{(\delta, r) \text {-irr }}(\mathcal{P})$ is allowed to have a bipartite graph $P_{a}^{i j}$ which is not $\left(\ell^{-1}, \varepsilon\right)$-regular (where $1 \leq i<j \leq t$ and $1 \leq a \leq \ell_{i j}$ ), while a triad $P \in \operatorname{Triad}_{\left(\alpha_{0}, \delta\right)-\text { exc }}(\mathcal{P})$ is not.)

Now, to prove Theorem 2.4 according to the outline from the Introduction, we need the following Propositions 3.2 and 3.4, and begin by presenting the former. Proposition 3.2 below generalizes the common implication of (2) and (3) (which said that, for graphs, $\varepsilon$-regularity implies $K_{2,2}^{(2)}$-minimality). Proposition 3.2 asserts that if $\mathcal{H}$ is $\left(\delta_{A}, r\right)$-regular w.r.t. $P$, then it is also $\left(\alpha, \delta_{B}\right)$-minimal w.r.t. $P$ (or, as we shall apply it, if $\mathcal{H}$ is $\left(\alpha, \delta_{B}\right)$-exceesive w.r.t. $P$, then it is also $\left(\delta_{A}, r\right)$-irregular w.r.t. $\left.P\right)$.

Proposition 3.2 (excessiveness $\Rightarrow$ irregularity). For all $\alpha$ and $\delta_{B}$, there exists $\delta_{A}>0$ so that for all integers $\ell$, there exist $\varepsilon>0$ and integer $r$ so that whenever $\mathcal{H}$ and $P$ satisfy the hypothesis of Setup 2.1 with constants $\alpha, \ell$ and $\varepsilon$ and $n$ sufficiently large, then the following holds:

(1) if $\mathcal{H}$ is $\left(\alpha, \delta_{B}\right)$-excessive with respect to $P$, then $\mathcal{H}$ is $\left(\delta_{A}, r\right)$-irregular with respect to $P$;

(2) moreover, one may construct, in time $O\left(n^{5}\right)$, a witness $\mathbf{Q}_{r}$ of the $\left(\delta_{A}, r\right)$-irregularity of $\mathcal{H}$ with respect to $P$. 
Remark 3.3. Statement (1) of Proposition 3.2 can be inferred from Lemma 3.1.1, the 'Counting Lemma', of [30]. Indeed, let $\mathcal{H}$ and $P$ be given as in Setup 2.1 with suitably chosen constants, where $\mathcal{H}$ is $(\delta, r)$-regular w.r.t. $P$. The Counting Lemma implies that $\mathcal{H}$ contains (asymptotically) the same number of copies of any fixed subhypergraph (and in particular, $K_{2,2,2}^{(3)}$ ) as is expected in the corresponding random 3-partite hypergraph. (Here, the corresponding random hypergraph is the binomial random subset of triangles from $P$ including each element of $\mathcal{K}_{3}^{(2)}(P)$ independently with probability $\alpha$.)

In this paper, we shall need Statement (2) of Proposition 3.2. This statement was proven, in slightly different language, in Lemma 5.8 (Algorithm A) of [11]. For completeness, we prove that Proposition 3.2 follows from Lemma 5.8 (Algorithm A) of [11], and give this proof in Section 7.

We remind the reader (cf. (8)) that the converse of Proposition 3.2 is, in general, not true (see [11] for details).

In our proof of the hypergraph regularity lemma, Theorem 2.4, we will also use Proposition 3.4 below. This proposition will extend the 'index-increasing' step (7) stated the Introduction from graphs to hypergraphs. To motivate this proposition, note that we may count the number of copies of $K_{2,2,2}^{(3)}$ spanned in each triad $P$ of an $(\ell, t, \gamma, \varepsilon)$-partition $\mathcal{P}$. For those triads $P$ with 'excessively many' copies, Proposition 3.2 asserts $P$ is 'irregular', and builds a corresponding witness $\mathbf{Q}_{P}$. If many of these triads $P$ are found, in this way, to be irregular, then the following proposition will construct a new partition $\mathcal{P}^{\prime}$ from $\mathcal{P}$ and the witnesses $\mathbf{Q}_{P}$, where $\mathcal{P}^{\prime}$ has index non-trivially larger than that of $\mathcal{P}$. (In what follows, one may therefore think of the subfamily $\mathcal{T}^{*}$ as a class of suitably 'excessive' triads.)

Proposition 3.4 (inflating the index). Let constants $\delta$ and $\gamma$ be given as well as functions $\varepsilon: \mathbb{N}^{+} \rightarrow(0,1)$ and $\boldsymbol{r}: \mathbb{N}^{+} \rightarrow \mathbb{N}^{+}$and integers $\ell_{\mathrm{old}}$ and $t_{\mathrm{old}}$. There exist constants

$$
L_{0}=L_{0}\left(\delta, \gamma, \varepsilon, \boldsymbol{r}, \ell_{\text {old }}, t_{\text {old }}\right), \quad T_{0}=T_{0}\left(\delta, \gamma, \varepsilon, \boldsymbol{r}, \ell_{\text {old }}, t_{\text {old }}\right), \quad N_{0}=N_{0}\left(\delta, \gamma, \varepsilon, \boldsymbol{r}, \ell_{\text {old }}, t_{\text {old }}\right)
$$

so that the following holds:

Suppose $\mathcal{G}$ is a 3-graph on $N>N_{0}$ vertices with $\left(\ell_{\mathrm{old}}, t_{\mathrm{old}}, \gamma, \varepsilon\left(\ell_{\mathrm{old}}\right)\right)$-partition $\mathcal{P}_{\mathrm{old}}$, and suppose $\mathcal{T}^{*}=\operatorname{Triad}_{\left(\delta, \boldsymbol{r}\left(\ell_{\mathrm{old}}\right)\right) \text {-irr }}^{*}\left(\mathcal{P}_{\text {old }}\right)$ is a subfamily of the collection of all $\left(\delta, \boldsymbol{r}\left(\ell_{\mathrm{old}}\right)\right)$-irregular triads $\operatorname{Triad}_{\left(\delta, \boldsymbol{r}\left(\ell_{\text {old }}\right)\right) \text {-irr }}\left(\mathcal{P}_{\text {old }}\right)$ (see the notation in (18)), satisfying the following properties:

(1) for each triad $P$ of the subfamily $\mathcal{T}^{*}$, one is given witness $\mathbf{Q}_{\boldsymbol{r}\left(\ell_{\mathrm{old}}\right), P}$ of the $\left(\delta, \boldsymbol{r}\left(\ell_{\mathrm{old}}\right)\right)$ irregularity of $\mathcal{G}_{P}=\mathcal{G} \cap \mathcal{K}_{3}^{(2)}(P)$ with respect to $P$;

$$
\sum_{P \in \mathcal{T}^{*}}\left|\mathcal{K}_{3}^{(2)}(P)\right| \geq \delta N^{3}
$$

Then,

(a) there exists an equitable $\left(\ell_{\text {new }}, t_{\text {new }}, \gamma, \varepsilon\left(\ell_{\text {new }}\right)\right)$-partition $\mathcal{P}_{\text {new }}$ of $\left(\begin{array}{c}V \\ 2\end{array}\right)$ for which

$$
\text { ind } \mathcal{P}_{\text {new }} \geq \text { ind } \mathcal{P}_{\text {old }}+\frac{\delta^{4}}{2}
$$

where $\ell_{\text {old }} \leq \ell_{\text {new }} \leq L_{0}$ and $t_{\text {old }} \leq t_{\text {new }} \leq T_{0}$. Moreover,

(b) there exists an algorithm which in time $O\left(N^{2}\right)$ constructs the partition $\mathcal{P}_{\text {new }}$ above from $\mathcal{P}_{\text {old }}$ and the given collection of witnesses $\left\{\mathbf{Q}_{\boldsymbol{r}\left(\ell_{\mathrm{old}}\right), P}: P \in \mathcal{T}^{*}\right\}$. 
The proof of Proposition 3.4 is given in [14] with no focus to being algorithmic. We shall not give a formal proof of Proposition 3.4, but we will now sketch a proof to indicate how its algorithmic part is obtained:

The approach in [14] is similar to Szemerédi's [45]. Consider the Venn diagram of the intersections of the witnesses $\mathbf{Q}_{P}=\mathbf{Q}_{\boldsymbol{r}\left(\ell_{\mathrm{old}}\right), P}$ over all $P \in \mathcal{T}^{*}$. In (1) of the hypothesis in Proposition 3.4, these witnesses are given to us. (In Szemerédi [45], these witnesses were subsets of vertices; here, the witnesses are ( $r$-tuples of) subsets of pairs.) This Venn diagram has at most

$$
2^{\left|\mathcal{T}^{*}\right| \boldsymbol{r}\left(\ell_{\text {old }}\right)} \leq 2^{t_{\text {old }}^{3} \ell_{\text {old }}^{3}} \boldsymbol{r}\left(\ell_{\text {old }}\right)
$$

regions (this number is independent of $N$ ), where each region is a bipartite graph. This Venn diagram defines a refinement $\mathcal{P}_{\text {old }}^{\prime}$ of $\mathcal{P}_{\text {old }}$, so that $\mathcal{P}_{\text {old }}^{\prime}$ is itself a partition. (The index of $\mathcal{P}_{\text {old }}^{\prime}$ is larger than that of $\mathcal{P}_{\text {old }}$ on account of the fact that in (2), we assumed 'many' triangles were lost to irregular triads in $\mathcal{P}_{\text {old }}$.) The bipartite graphs of $\mathcal{P}_{\text {old }}^{\prime}$ may not be 'regular', so we apply Szemerédi's Regularity Lemma to each. (The Regularity Lemma is known to be algorithmic by the result of Alon et al. $[1,13]$.) The resulting (regular) bipartite graphs may have differing densities, so we 'randomly slice' each into thinner 'equidense' layers (this is the same idea we discussed earlier after stating Theorem 2.5). This random slicing is derandomized, however, in Lemma 6 (p. 17) of Haxell and Rödl [22]. (These latter two refinements of $\mathcal{P}_{\text {old }}^{\prime}$ are done at no real cost to the index of $\mathcal{P}_{\text {old }}^{\prime}$.) The process above produces the partition $\mathcal{P}_{\text {new }}$. For the formal details of this outline, see Lemmas 3.9 and 3.10 of [14] (pp. 145 and 149) and Lemma 6 of [22].

We now give the proof of Theorem 2.4 using Propositions 3.2 and 3.4 and following the outline of the Introduction.

3.1. Proof of Theorem 2.4. For quick reference on the proof that follows, we provide a flowchart in Figure 1. In the immediate sequel, we formally describe all parameters used in our argument.

3.1.1. Constants of Theorem 2.4. Let $\alpha_{0}, \delta, \gamma>0$ be given as well as function $\varepsilon: \mathbb{N} \rightarrow(0,1)$. For simplicity, let $\ell_{0}=1$ (most applications of the original Frankl-Rödl Hypergraph Regularity Lemma (cf. Theorem 3.5 of [14]) take $\ell_{0}=1$ ). Let integer $t_{0}$ be given. Let us now briefly describe a few auxiliary constants we will need momentarily.

For $\alpha_{0}$ and $\delta_{B}=\delta$, let

$$
\delta_{A}^{(3.2)}=\delta_{A}^{(3.2)}\left(\alpha_{0}, \delta\right)
$$

be the constant guaranteed by Proposition 3.2 where we may assume, without loss of generality, that

$$
\delta_{A}^{(3.2)} \leq \delta
$$

For an integer variable $\ell \in \mathbb{N}$, let

$$
\varepsilon^{(3.2)}(\ell)=\varepsilon^{(3.2)}\left(\alpha_{0}, \delta, \delta_{A}^{(3.2)}, \ell\right) \quad \text { and } \quad r^{(3.2)}(\ell)=r^{(3.2)}\left(\alpha_{0}, \delta, \delta_{A}^{(3.2)}, \ell\right)
$$

be the functions guaranteed by Proposition 3.2. Without loss of generality, we shall assume the given function $\varepsilon$ satisfies

$$
\varepsilon(\ell) \leq \varepsilon^{(3.2)}(\ell)
$$

for every integer $\ell \in \mathbb{N}$.

Theorem 2.4 promises constants $L_{0}, T_{0}$ and $N_{0}$, which we shall now formally describe (although, they will be easier to see later in context). With $\gamma>0$ (given as above), $\delta_{A}^{(3.2)}$ (given 
in (20)), function $\varepsilon(\ell) \leq \varepsilon^{(3.2)}(\ell)$ (given as in (23)) and $r^{(3.2)}(\ell)$ (given in (22)) fixed, and for arbitrary integers $\ell_{\text {old }}$ and $t_{\text {old }}$, Proposition 3.4 promises constants

$$
\begin{aligned}
& L_{0}\left(\ell_{\text {old }}, t_{\text {old }}\right)=L_{0}^{(3.4)}\left(\gamma, \delta_{A}^{(3.2)}, \varepsilon(\ell), r^{(3.2)}(\ell), \ell_{\text {old }}, t_{\text {old }}\right), \\
& T_{0}\left(\ell_{\text {old }}, t_{\text {old }}\right)=T_{0}^{(3.4)}\left(\gamma, \delta_{A}^{(3.2)}, \varepsilon(\ell), r^{(3.2)}(\ell), \ell_{\text {old }}, t_{\text {old }}\right),
\end{aligned}
$$

and

$$
N_{0}\left(\ell_{\text {old }}, t_{\text {old }}\right)=N_{0}^{(3.4)}\left(\gamma, \delta_{A}^{(3.2)}, \varepsilon(\ell), r^{(3.2)}(\ell), \ell_{\text {old }}, t_{\text {old }}\right) .
$$

We successively define constants $L_{0}^{(i)}, T_{0}^{(i)}$ and $N_{0}^{(i)}, 0 \leq i \leq 2 /\left(\delta_{A}^{(3.2)}\right)^{4}$, as follows: with already given constants $\ell_{0}=1$ and $t_{0}$, set

$$
L_{0}^{(0)}=L_{0}\left(\ell_{0}=1, t_{0}\right), \quad T_{0}^{(0)}=T_{0}\left(\ell_{0}=1, t_{0}\right), \quad N_{0}^{(0)}=N_{0}\left(\ell_{0}=1, t_{0}\right) .
$$

For $1 \leq i \leq 2 /\left(\delta_{A}^{(3.2)}\right)^{4}$, set

$$
L_{0}^{(i)}=L_{0}\left(L_{0}^{(i-1)}, T_{0}^{(i-1)}\right), \quad T_{0}^{(i)}=T_{0}\left(L_{0}^{(i-1)}, T_{0}^{(i-1)}\right), \quad N_{0}^{(i)}=N_{0}\left(L_{0}^{(i-1)}, T_{0}^{(i-1)}\right) .
$$

Then, the constants $L_{0}, T_{0}$ and $N_{0}$ of Theorem 2.4 are given by

$$
L_{0}=L_{0}^{\left(i_{*}\right)}, \quad T_{0}=T_{0}^{\left(i_{*}\right)}, \quad N_{0}=N_{0}^{\left(i_{*}\right)}
$$

where

$$
i_{*}=\left\lfloor\frac{2}{\left(\delta_{A}^{(3.2)}\right)^{4}}\right\rfloor .
$$

This concludes our discussion of the constants.

3.1.2. The argument. Let 3-graph $\mathcal{G}$ be given on sufficiently large vertex set $V,|V|=N$. We are going to construct, in time $O\left(N^{6}\right)$, an $\left(\alpha_{0}, \delta\right)$-minimal and equitable $(\ell, t, \gamma, \varepsilon(\ell))$ partition $\mathcal{P}$ for $\mathcal{G}$ for which $1 \leq \ell \leq L_{0}$ and $t_{0} \leq t \leq T_{0}$ for $L_{0}$ and $T_{0}$ given in (25). The main idea of the proof is outlined in the Introduction as well as the flow-chart in Figure 1.

Start with the partition $\mathcal{P}_{1}$, taken as $K\left(V_{0}, V_{1}, \ldots, V_{t_{0}}\right)$ where $V(\mathcal{G})=V_{0} \cup V_{1} \cup \cdots \cup V_{t_{0}}$ is any vertex partition with $\left|V_{0}\right|<t_{0}$ and $\left|V_{1}\right|=\cdots=\left|V_{t_{0}}\right|$ (so that $\mathcal{B}$ consists of the $\left(\begin{array}{c}t_{0} \\ 2\end{array}\right)$ complete bipartite graphs $\left.K\left[V_{i}, V_{j}\right], 1 \leq i<j \leq t_{0}\right)$. Then $\mathcal{P}_{1}$ is an equitable $\left(\ell_{0}=1, t_{0}, \gamma, \varepsilon\left(\ell_{0}\right)\right)$ partition since, in fact, it is an equitable $\left(\ell_{0}=1, t_{0}, 0, \varepsilon\right)$-partition for any $\varepsilon>0$.

For an integer $1 \leq s<2 /\left(\delta_{A}^{(3.2)}\right)^{4}$ (this upper bound will become clearer within the context of the proof), assume $\mathcal{P}_{1}, \ldots, \mathcal{P}_{s}$ are constructed partitions where $\mathcal{P}_{s}$ is an equitable $\left(\ell_{s}, t_{s}, \gamma, \varepsilon\left(\ell_{s}\right)\right)$-partition of $\left(\begin{array}{c}V \\ 2\end{array}\right)$ where

$$
1 \leq \ell_{s} \leq L_{0}^{(s-1)} \quad \text { and } \quad t_{0} \leq t_{s} \leq T_{0}^{(s-1)}
$$

for the constants $L_{0}^{(s-1)}$ and $T_{0}^{(s-1)}$ defined in (24). The main idea here is similar to that used by Szemerédi [45]. We shall either verify that $\mathcal{P}_{s}$ is $\left(\alpha_{0}, \delta\right)$-minimal, or else, we shall construct a new partition $\mathcal{P}_{s+1}$ whose index (cf. (17)) is larger than that of $\mathcal{P}_{s}$. Moreover, we show that these steps can be carried out in time $O\left(N^{6}\right)$.

Our first step in the algorithm is to compute, for the partition $\mathcal{P}_{s}$ above, the sum (cf. (15))

$$
\sum_{P \in \operatorname{Triad}_{\left(\alpha_{0}, \delta\right)-\operatorname{exc}}\left(\mathcal{P}_{s}\right)}\left|\mathcal{K}_{3}^{(2)}(P)\right| .
$$

The central operation in computing this sum consists of identifying

$$
\mathcal{T}_{\text {exc }} \stackrel{\text { def }}{=} \operatorname{Triad}_{\left(\alpha_{0}, \delta\right)-\operatorname{exc}}\left(\mathcal{P}_{s}\right) \subseteq \operatorname{Triad}\left(\mathcal{P}_{s}\right) .
$$


Indeed, for each of the $\left(\begin{array}{c}t_{s} \\ 3\end{array}\right) \ell_{s}^{3}$ triads $P \in \operatorname{Triad}\left(\mathcal{P}_{s}\right)$, we count the number of $K_{2,2,2}^{(3)}$ 's appearing in $\mathcal{G}_{P}=\mathcal{G} \cap \mathcal{K}_{3}^{(2)}(P)$. As such, computing (27) has complexity $O\left(N^{6}\right)$.

Upon computing the sum in (27), two outcomes can occur (cf. (28)):

$$
\sum_{P \in \mathcal{T}_{\text {exc }}}\left|\mathcal{K}_{3}^{(2)}(P)\right|<\delta N^{3} \quad \stackrel{(15)}{\Longrightarrow} \quad \mathcal{P}_{s} \text { is }\left(\alpha_{0}, \delta\right) \text {-minimal }
$$

or

$$
\sum_{P \in \mathcal{T}_{\text {exc }}}\left|\mathcal{K}_{3}^{(2)}(P)\right| \geq \delta N^{3} \quad \stackrel{(15)}{\Longrightarrow} \quad \mathcal{P}_{s} \text { is }\left(\alpha_{0}, \delta\right) \text {-excessive. }
$$

If we determine that $(29)$ occurs, then we are done. Indeed, $\mathcal{P}_{s}$ is the $\left(\alpha_{0}, \delta\right)$-minimal and equitable $\left(\ell_{s}, t_{s}, \gamma, \varepsilon\left(\ell_{s}\right)\right)$-partition we wanted to construct. This situation is the analogue of Step $\left(1^{\prime}\right)$ in the Introduction.

Otherwise, we determine that (30) occurs and we are in a situation similar to Step $\left(2^{\prime}\right)$ in the outline of the Introduction where we increased the graph index in (7). Here, we want to show that from the large sum in (30), we may construct a new and equitable $\left(\ell_{s+1}, t_{s+1}, \gamma, \varepsilon\left(\ell_{s+1}\right)\right)$ partition $\mathcal{P}_{s+1}$ of $\left(\begin{array}{c}V \\ 2\end{array}\right)$ whose index is non-trivially larger than ind $\mathcal{P}_{s}$. Moreover, we want to show the new parameters $\ell_{s+1}$ and $t_{s+1}$ satisfy $\ell_{s+1} \leq L_{0}^{(s)}$ and $t_{s+1} \leq T_{0}^{(s)}$ (cf. (24)). Proposition 3.4 is precisely the tool to do this.

Before we can apply Proposition 3.4 to the partition $\mathcal{P}_{s}$, we need to show that its hypothesis is met. To that end, set

$$
\mathcal{T}^{*}=\operatorname{Triad}_{\left(\delta_{A}^{(3.2)}, r^{(3.2)}\left(\ell_{s}\right)\right) \text {-irr }}^{*}\left(\mathcal{P}_{s}\right)=\operatorname{Triad}_{\left(\alpha_{0}, \delta\right) \text {-exc }}\left(\mathcal{P}_{s}\right)=\mathcal{T}_{\text {exc }}
$$

Proposition 3.2 will guarantee that for each excessive triad $P \in \mathcal{T}_{\text {exc }}$,

$$
\begin{aligned}
& \text { one may construct, in time } O\left(\left(N / t_{s}\right)^{5}\right) \text {, a witness } \mathbf{Q}_{\boldsymbol{r}\left(\ell_{s}\right), P} \\
& \qquad \text { of the }\left(\delta_{A}^{(3.2)}, r^{(3.2)}\left(\ell_{s}\right)\right) \text {-irregularity of } \mathcal{G}_{P}=\mathcal{G} \cap \mathcal{K}_{3}^{(2)}(P) \text { w.r.t. } P,
\end{aligned}
$$

so that, in particular (cf. (18)),

$$
\mathcal{T}^{*}=\mathcal{T}_{\text {exc }} \subseteq \operatorname{Triad}_{\left(\delta_{A}^{(3.2)}, r^{(3.2)}\left(\ell_{s}\right)\right)-\text { irr }}\left(\mathcal{P}_{s}\right) .
$$

Indeed, fix triad $P=P_{a}^{i j} \cup P_{b}^{j k} \cup P_{c}^{i k} \in \operatorname{Triad}_{\left(\alpha_{0}, \delta\right) \text {-exc }}\left(\mathcal{P}_{s}\right)$ where $1 \leq i<j<k \leq t_{s}$ and $1 \leq$ $a, b, c \leq \ell_{s}$ (so that $P$ has 3-partition $V_{i} \cup V_{j} \cup V_{k}$, where $\left\lfloor N / t_{s}\right\rfloor \leq\left|V_{i}\right|,\left|V_{j}\right|,\left|V_{k}\right| \leq\left\lceil N / t_{s}\right\rceil$ ). We intend to apply Proposition 3.2 to graph $P$ and hypergraph $\mathcal{H}=\mathcal{G}_{P}=\mathcal{G} \cap \mathcal{K}_{3}(P)$. Note that the constants involved with $P$ and $\mathcal{G}_{P}$ meet the requirements of Proposition 3.2. Indeed, $P \in \mathcal{T}_{\text {exc }}$ means that with $\delta_{B}=\delta, \mathcal{G}_{P}$ is $\left(\alpha_{P}, \delta_{B}\right)$-excessive w.r.t. $P$ where $\alpha_{P}=\left|\mathcal{G}_{P}\right| /\left|\mathcal{K}_{3}^{(2)}(P)\right| \geq$ $\alpha_{0}$ where $\alpha_{0}$ was given in the beginning of the proof (cf. Definition 2.2 and Remark 2.3). Moreover, each of the bipartite graphs $P_{a}^{i j}, P_{b}^{j k}, P_{c}^{i k}$ is $\left(1 / \ell_{s}, \varepsilon\left(\ell_{s}\right)\right)$-regular, where by (23), $\varepsilon\left(\ell_{s}\right) \leq \varepsilon^{(3.2)}\left(\ell_{s}\right)$ where $\varepsilon^{(3.2)}\left(\ell_{s}\right)$ is chosen in $(22)$ sufficiently small to apply Proposition 3.2. Therefore, Statement (2) of Proposition 3.2 guarantees a $O\left(\left(N / t_{s}\right)^{5}\right)$-time algorithm which constructs a witness $\mathbf{Q}_{r^{(3.2)}\left(\ell_{s}\right), P}$ of the $\left(\delta_{A}^{(3.2)}, r^{(3.2)}\left(\ell_{s}\right)\right)$-irregularity of $\mathcal{G}_{P}$ w.r.t. $P$.

Continuing, (32) implies that Statement (1) in the hypothesis of Proposition 3.4 is met by the partition $\mathcal{P}_{s}$. To see that Statement (2) in the hypothesis of Proposition 3.4 is also met, we 


\begin{tabular}{|c|c|c|}
\hline compute $\sum_{P \in \mathcal{T}_{\text {exc }}}\left|\mathcal{K}_{3}^{(2)}(P)\right|$ & $\stackrel{\text { if }}{\longrightarrow}$ & $\sum_{P \in \mathcal{T}_{\text {exc }}}\left|\mathcal{K}_{3}^{(2)}(P)\right|<\delta N^{3}$ \\
\hline \multicolumn{2}{|l|}{ if } & then \\
\hline $\begin{array}{l}\sum_{P \in \mathcal{T}_{\text {exc }}}\left|\mathcal{K}_{3}^{(2)}(P)\right|>\delta N^{3} \\
\left(\mathcal{P}_{s} \text { is 'excessive') }\right.\end{array}$ & & $\mathcal{P}_{s}$ is $\left(\alpha_{0}, \delta\right)$-minimal; stop \\
\hline \multicolumn{3}{|l|}{ Prop. 3.2} \\
\hline $\begin{array}{l}\forall P \in \mathcal{T}_{\text {exc }}, \text { construct wit- } \\
\text { ness } \mathbf{Q}_{r^{(3.2)}\left(\ell_{s}\right), P} \in \mathcal{Q}_{\text {exc }} \text { of } \\
\text { irregularity }\end{array}$ & \multirow[b]{3}{*}{$\stackrel{\text { Prop. } 3.4}{\longrightarrow}$} & repeat process for $\mathcal{P}_{s+1}$. \\
\hline so that & & \\
\hline $\begin{array}{l}\text { triads } \mathcal{T}^{*}=\mathcal{T}_{\text {exc }} \text { satisfy hypothe- } \\
\text { sis of Prop. } 3.4\end{array}$ & & $\begin{array}{l}\text { construct partition } \mathcal{P}_{s+1} \text { where } \\
\quad \text { ind } \mathcal{P}_{s+1} \geq \operatorname{ind} \mathcal{P}_{s}+\frac{\left(\delta_{A}^{(3.2)}\right)^{4}}{2}\end{array}$ \\
\hline
\end{tabular}

Figure 1. Flow chart for the proof of Theorem 2.4. Here, $\mathcal{P}_{s}$ is a given $\left(\ell_{s}, t_{s}, \gamma, \varepsilon\left(\ell_{s}\right)\right)$-partition, $\delta_{A}^{(3.2)} \leq \delta(\mathrm{cf} .(21))$ and $\mathcal{T}^{*}=\mathcal{T}_{\text {exc }}$ is defined in $(28)$.

return to the large sum in $(30)($ cf. $(31))$ to see

$$
\sum_{P \in \mathcal{T}^{*}}\left|\mathcal{K}_{3}^{(2)}(P)\right| \geq \delta N^{3} \stackrel{(21)}{\geq} \delta_{A}^{(3.2)} N^{3} .
$$

Thus, Proposition 3.4 applies to the partition $\mathcal{P}_{s}$.

Proposition 3.4 constructs, in time $O\left(N^{2}\right)$, an equitable $\left(\ell_{s+1}, t_{s+1}, \gamma, \varepsilon\left(\ell_{s+1}\right)\right)$-partition $\mathcal{P}_{s+1}$ of $\left(\begin{array}{c}V \\ 2\end{array}\right)$ for which

$$
\text { ind } \mathcal{P}_{s+1} \geq \text { ind } \mathcal{P}_{s}+\frac{\left(\delta_{A}^{(3.2)}\right)^{4}}{2}
$$

and for which

$$
\ell_{s+1} \leq L_{0}\left(\ell_{s}, t_{s}\right) \stackrel{(26)}{\leq} L_{0}\left(L_{0}^{(s-1)}, T_{0}^{(s-1)}\right) \stackrel{(24)}{=} L_{0}^{(s)}
$$

and

$$
t_{0} \leq t_{s+1} \leq T_{0}\left(\ell_{s}, t_{s}\right) \stackrel{(26)}{\leq} T_{0}\left(L_{0}^{(s-1)}, T_{0}^{(s-1)}\right) \stackrel{(24)}{=} T_{0}^{(s)}
$$

Now, the proof of Theorem 2.4 is essentially complete. Indeed, by (33), we can repeat the constructive procedure above at most $2 /\left(\delta_{A}^{(3.2)}\right)^{4}$ times (the index can't exceed one), in which case, for some iteration, we must have constructed an $\left(\alpha_{0}, \delta\right)$-minimal and equitable $(\ell, t, \gamma, \varepsilon(\ell))$ partition $\mathcal{P}$, where $\ell \leq L_{0}$ and $t_{0} \leq t \leq T_{0}$ for $L_{0}$ and $T_{0}$ defined in (25).

As a final note, it is easy to see that the construction above is completed in time $O\left(N^{6}\right)$. Indeed, the application of Proposition 3.2, which constructs witnesses $\mathbf{Q}_{r^{(3.2)}\left(\ell_{s}\right), P}$ for each $P \in \mathcal{T}^{*}=\mathcal{T}_{\text {exc }}$, contributes $O\left(N^{5}\right)$ complexity. The application of Proposition 3.4, which constructs the new partition $\mathcal{P}_{s+1}$, contributes $O\left(N^{2}\right)$ complexity. As such, the complexity 
$O\left(N^{6}\right)$ of the algorithm is generated by counting copies of $K_{2,2,2}^{(3)}$ in (27) (by which we determine whether or not partitions $\mathcal{P}$ are $\left(\alpha_{0}, \delta\right)$-minimal). This completes the proof of Theorem 2.4.

\section{Properties of $(\alpha, \delta)$-minimality}

It is well known that, in certain hypergraph contexts, having the (asymptotic) minimum number of copies of $K_{2,2,2}^{(3)}$ implies various other properties (see [7, 25]). In our proof of the Counting Lemma, Theorem 2.7, we shall need some of these properties for the context of Setup $2.1^{1}$ (these properties are stated as upcoming Propositions 4.1-4.6). At the end of this section, we sketch the proofs of upcoming Propositions 4.1-4.6.

Recall that we asserted in (13) that a hypergraph $\mathcal{H}$ (with graph $P$ ) as in Setup 2.1 will contain at least $\sim\left(\alpha^{8} / \ell^{12}\right)\left(\begin{array}{c}n \\ 2\end{array}\right)^{3}$ many copies of $K_{2,2,2}^{(3)}$. If $\mathcal{H}$ has at most $\sim\left(\alpha^{8} / \ell^{12}\right)\left(\begin{array}{l}n \\ 2\end{array}\right)^{3}$ many such copies, then we defined (in Definition 2.2) $\mathcal{H}$ to be $(\alpha, \delta)$-minimal w.r.t. $P$. In this section, we also wish to consider a notion of minimality for the frequency of the subhypergraph $K_{1,2,2}^{(3)}$ where $K_{1,2,2}^{(3)}$ is the complete 3-uniform 3-partite hypergraph whose vertex partition classes have sizes 1, 2 and 2. To that end, with $\mathcal{H}$ and $P$ given as in Setup 2.1, let

$$
\begin{aligned}
\mathcal{K}_{1,2,2}^{(3)}(\mathcal{H})= & \left\{(\{x\},\{a, b\},\{u, v\}): x \in V_{1},\{a, b\} \in\left(\begin{array}{c}
V_{2} \\
2
\end{array}\right),\{u, v\} \in\left(\begin{array}{c}
V_{3} \\
2
\end{array}\right),\right. \\
& \left.\{x\} \cup\{a\} \cup\{b\} \cup\{u\} \cup\{v\} \text { induces } K_{1,2,2}^{(3)} \text { in } \mathcal{H}\right\} .
\end{aligned}
$$

Note that this family is different from, say,

$$
\begin{aligned}
\mathcal{K}_{2,2,1}^{(3)}(\mathcal{H})= & \left\{(\{x, y\},\{a, b\},\{u\}):\{x, y\} \in\left(\begin{array}{c}
V_{1} \\
2
\end{array}\right),\{a, b\} \in\left(\begin{array}{c}
V_{2} \\
2
\end{array}\right), u \in V_{3},\right. \\
& \left.\{x\} \cup\{y\} \cup\{a\} \cup\{b\} \cup\{u\} \text { induces } K_{2,2,1}^{(3)} \text { in } \mathcal{H}\right\},
\end{aligned}
$$

and different from $\mathcal{K}_{2,1,2}^{(3)}(\mathcal{H})$, which is defined similarly. We proceed to state Propositions 4.14.6 , and conclude this section with (sketches of) their proofs.

Proposition 4.1. With given constants $\alpha, \delta$ and $\ell$, sufficiently small $\varepsilon=\varepsilon(\alpha, \delta, \ell)>0$ and sufficiently large $n=n(\alpha, \delta, \ell, \varepsilon)$, suppose $\mathcal{H}$ and $P$ are as in Setup 2.1. Then

$$
\left|\mathcal{K}_{1,2,2}^{(3)}(\mathcal{H})\right| \geq \frac{\alpha^{4}}{\ell^{8}} n\left(\begin{array}{l}
n \\
2
\end{array}\right)^{2}\left(1-\varepsilon^{1 / 10}\right) \quad \text { and } \quad\left|\mathcal{K}_{2,2,2}^{(3)}(\mathcal{H})\right| \geq \frac{\alpha^{8}}{\ell^{12}}\left(\begin{array}{l}
n \\
2
\end{array}\right)^{3}\left(1-\varepsilon^{1 / 10}\right) .
$$

The following definition is now motivated (cf. Definition 2.2).

Definition 4.2. With $\mathcal{H}$ and $P$ given as in Setup 2.1, we say $\mathcal{H}$ is $(\alpha, \delta)_{1}$-minimal w.r.t. $P$ if

$$
\left|\mathcal{K}_{1,2,2}^{(3)}(\mathcal{H})\right| \leq \frac{\alpha^{4}}{\ell^{8}} n\left(\begin{array}{l}
n \\
2
\end{array}\right)^{2}(1+\delta) .
$$

Remark 4.3. For a clear distiction in this section, we shall refer to the original $(\alpha, \delta)$-minimality as $(\alpha, \delta)_{2}$-minimality.

The following proposition relates $(\alpha, \delta)_{2}$-minimality with $(\alpha, \delta)_{1}$-minimality.

\footnotetext{
${ }^{1}$ Setup 2.1 allows for the possibility that $1 / \ell \ll \delta$, while the opposite situation $\delta \ll 1 / \ell$ is considered in $[7,25]$. The proofs we sketch for Propositions 4.1-4.6 are similar, nonetheless, to the proofs given in [7, 25].
} 
Proposition 4.4. With given constants $\alpha, \delta$ and $\ell$, sufficiently small $\varepsilon=\varepsilon(\alpha, \delta, \ell)>0$ and sufficiently large $n=n(\alpha, \delta, \ell, \varepsilon)$, suppose $\mathcal{H}$ and $P$ are as in Setup 2.1. If $\mathcal{H}$ is $(\alpha, \delta)_{2}$-minimal w.r.t. $P$, then $\mathcal{H}$ is also $(\alpha, \delta)_{1}$-minimal w.r.t. $P$. In particular, if $\mathcal{H}$ is $(\alpha, \delta)_{2}$-minimal w.r.t. $P$, then all of the following inequalities hold:

$$
\left|\mathcal{K}_{1,2,2}^{(3)}(\mathcal{H})\right|,\left|\mathcal{K}_{2,1,2}^{(3)}(\mathcal{H})\right|,\left|\mathcal{K}_{2,2,1}^{(3)}(\mathcal{H})\right| \leq \frac{\alpha^{4}}{\ell^{8}} n\left(\begin{array}{l}
n \\
2
\end{array}\right)^{2}(1+\delta)
$$

We sketch the proof of Proposition 4.4 at the end of this section.

To present Propositions 4.5 and 4.6, we require some notation. With $\mathcal{H}$ and $P$ given as in Setup 2.1 and $x \in V$ an arbitrary vertex, let

$$
L_{x}=\{\{u, v\} \in P:\{x, u, v\} \in \mathcal{H}\}
$$

denote the link of $x$. For vertices $x, y \in V$, let

$$
L_{x y}=L_{x} \cap L_{y}
$$

denote the colink of $x$ and $y$. For Propositions 4.5 and 4.6, we also consider the following supplemental notation. For $x \in V$, let

$$
P_{x}=\left\{\{u, v\} \in P:\{x, u, v\} \in \mathcal{K}_{3}^{(2)}(P)\right\}=P\left[N_{P}(x)\right]
$$

be the subgraph of $P$ induced on the $P$-neighborhood of $x$. For vertices $x, y \in V_{1}$, let

$$
P_{x y}=P_{x} \cap P_{y}=P\left[N_{P}(x, y)\right] .
$$

In the context of Setup 2.1, it is well known (cf. Fact 1.4 from [29]) from the $\left(\ell^{-1}, \varepsilon\right)$-regularity of $P^{12}, P^{23}$ and $P^{13}$ that all but $4 \varepsilon n$ vertices $x \in V_{1}\left(8 \varepsilon\left(\begin{array}{c}n \\ 2\end{array}\right)\right.$ pairs $\left.\{x, y\} \in\left(\begin{array}{c}V_{1} \\ 2\end{array}\right)\right)$ satisfy

$$
\begin{aligned}
\operatorname{deg}_{P^{1 j}}(x)=\left|N_{P^{1 j}}(x)\right|= & \left(\frac{1}{\ell} \pm \varepsilon\right) n, \quad j=2,3 \\
& \left(\operatorname{deg}_{P^{1 j}}(x, y)=\left|N_{P^{1 j}}(x, y)\right|=\left(\frac{1}{\ell} \pm \varepsilon\right)^{2} n, \quad j=2,3\right) .
\end{aligned}
$$

As such, with $\varepsilon$ sufficiently small (say, $0<\varepsilon \leq 1 /(2 \ell)^{2}$ ), all but $4 \varepsilon n$ vertices $x \in V_{1}\left(8 \varepsilon\left(\begin{array}{l}n \\ 2\end{array}\right)\right.$ pairs $\left.\{x, y\} \in\left(\begin{array}{c}V_{1} \\ 2\end{array}\right)\right)$ satisfy

$$
\begin{aligned}
\left|P_{x}\right|=\left(\frac{1}{\ell} \pm \varepsilon\right) \operatorname{deg}_{P^{12}}(x) \cdot \operatorname{deg}_{P^{13}}(x)=\left(\frac{1}{\ell} \pm \varepsilon\right)^{3} n^{2}=\frac{n^{2}}{\ell^{3}}(1 \pm 4 \ell \varepsilon) \\
\quad\left(\left|P_{x y}\right|=\left(\frac{1}{\ell} \pm \varepsilon\right) \operatorname{deg}_{P^{12}}(x, y) \cdot \operatorname{deg}_{P^{13}}(x, y)=\left(\frac{1}{\ell} \pm \varepsilon\right)^{5} n^{2}=\frac{n^{2}}{\ell^{5}}(1 \pm 6 \ell \varepsilon)\right)
\end{aligned}
$$

which follows from the $\left(\ell^{-1}, \varepsilon\right)$-regularity of $P^{23}$. In particular, one can prove (cf. Fact 1.5 from [29]) that all but $4 \varepsilon n$ vertices $x \in V_{1}$ (pairs $\{x, y\} \in\left(\begin{array}{c}V_{1} \\ 2\end{array}\right)$ ) satisfy that

$$
P_{x} \text { is }\left(\ell^{-1}, 2 \ell \varepsilon\right) \text {-regular }\left(P_{x y} \text { is }\left(\ell^{-1}, 4 \ell^{2} \varepsilon\right) \text {-regular }\right) .
$$

We now present Proposition 4.5.

Proposition 4.5. With given constants $\alpha, \delta$ and $\ell$, sufficiently small $\varepsilon=\varepsilon(\alpha, \delta, \ell)>0$ and sufficiently large $n=n(\alpha, \delta, \ell, \varepsilon)$, whenever $\mathcal{H}$ and $P$ are as in Setup 2.1, the following hold: 
(1) if $\mathcal{H}$ is $(\alpha, \delta)_{1}$-minimal w.r.t. $P$, then all but $3 \delta^{1 / 3} n$ vertices $x \in V_{1}$ satisfy

$$
\left|L_{x}\right|=\frac{\alpha}{\ell^{3}} n^{2}\left(1 \pm 3 \delta^{1 / 3}\right) \stackrel{(40)}{=} \alpha\left|P_{x}\right|\left(1 \pm 4 \delta^{1 / 3}\right) \text {. }
$$

(2) if $\mathcal{H}$ is $(\alpha, \delta)_{2}$-minimal w.r.t. $P$, then all but $3 \delta^{1 / 3} n^{2}$ pairs $\{x, y\} \in\left(\begin{array}{c}V_{1} \\ 2\end{array}\right)$ satisfy

$$
\left|L_{x y}\right|=\frac{\alpha^{2}}{\ell^{5}} n^{2}\left(1 \pm 3 \delta^{1 / 3}\right) \stackrel{(40)}{=} \alpha^{2}\left|P_{x y}\right|\left(1 \pm 4 \delta^{1 / 3}\right) .
$$

Upcoming Proposition 4.6 establishes a 'local characterization' of $(\alpha, \delta)_{1}$-minimality and $(\alpha, \delta)_{2}$-minimality, respectively. We use the following supplemental notation in our presentation. For a pair $1 \leq i<j \leq 3$ and integers $s_{1}, s_{2} \in\{1,2\}$, let

$$
\mathcal{K}_{s_{1}, s_{2}}^{(2)}\left(P^{i j}\right)=\left\{\left(S_{1}, S_{2}\right): S_{1} \in\left(\begin{array}{c}
V_{i} \\
s_{1}
\end{array}\right), S_{2} \in\left(\begin{array}{c}
N_{P^{i j}}\left(S_{1}\right) \\
s_{2}
\end{array}\right)\right\} .
$$

Note that for each element $\left(S_{1}, S_{2}\right) \in \mathcal{K}_{s_{1}, s_{2}}^{(2)}\left(P^{i j}\right)$, the set $S_{1} \cup S_{2}$ induces a copy of the complete bipartite graph $K_{s_{1}, s_{2}}^{(2)}$ in $P^{i j}$. In context, we shall use the standard fact that for $s_{1}, s_{2} \in\{1,2\}$,

$$
\left|\mathcal{K}_{s_{1}, s_{2}}^{(2)}\left(P^{i j}\right)\right|=\frac{1}{\ell^{s_{1} s_{2}}}\left(\begin{array}{l}
n \\
s_{1}
\end{array}\right)\left(\begin{array}{c}
n \\
s_{2}
\end{array}\right)\left(1 \pm \varepsilon^{1 / 2}\right)
$$

which follows from the the $\left(\ell^{-1}, \varepsilon\right)$-regularity of $P^{i j}$ (cf. Setup 2.1) whenever $\varepsilon>0$ is sufficiently small and $n=n(\ell, \varepsilon)$ is sufficiently large.

Proposition 4.6. With given constants $\alpha, \delta$ and $\ell$, sufficiently small $\varepsilon=\varepsilon(\alpha, \delta, \ell)>0$ and sufficiently large integer $n=n(\alpha, \delta, \ell, \varepsilon)$, whenever $\mathcal{H}$ and $P$ are as in Setup 2.1 with these constants, the following hold:

(1) (a) if $\mathcal{H}$ is $(\alpha, \delta)_{1}$-minimal with respect to $P$, then all but $3 \delta^{1 / 3}\left|\mathcal{K}_{1,2}^{(2)}\left(P^{12}\right)\right|$ many elements $(\{x\},\{a, b\}) \in \mathcal{K}_{1,2}^{(2)}\left(P^{12}\right)$ satisfy

$$
\operatorname{deg}_{L_{x}}(a, b)=\left(\frac{\alpha}{\ell}\right)^{2} \frac{n}{\ell}\left(1 \pm 3 \delta^{1 / 3}\right) \stackrel{(39)}{=}\left(\frac{\alpha}{\ell}\right)^{2} \operatorname{deg}_{P^{13}}(x)\left(1 \pm 6 \delta^{1 / 3}\right)
$$

conversely,

(b) if all but $\delta\left|\mathcal{K}_{1,2}^{(2)}\left(P^{12}\right)\right|$ many elements $(\{x\},\{a, b\}) \in \mathcal{K}_{1,2}^{(2)}\left(P^{12}\right)$ satisfy

$$
\operatorname{deg}_{L_{x}}(a, b)=\left(\frac{\alpha}{\ell}\right)^{2} \frac{n}{\ell}(1 \pm \delta)
$$

then $\mathcal{H}$ is $\left(\alpha, 3 \delta^{1 / 3}\right)_{1}$-minimal w.r.t. $P$;

(2) (a) if $\mathcal{H}$ is $(\alpha, \delta)_{2}$-minimal with respect to $P$, then all but $3 \delta^{1 / 3}\left|\mathcal{K}_{2,2}^{(2)}\left(P^{12}\right)\right|$ many elements $(\{x, y\},\{a, b\}) \in \mathcal{K}_{2,2}^{(2)}\left(P^{12}\right)$ satisfy

$$
\begin{aligned}
& \operatorname{deg}_{L_{x y}}(a, b)=\left(\frac{\alpha^{2}}{\ell}\right)^{2} \frac{n}{\ell^{2}}\left(1 \pm 3 \delta^{1 / 3}\right) \stackrel{(39)}{=}\left(\frac{\alpha^{2}}{\ell}\right)^{2} \operatorname{deg}_{P^{13}}(x, y)\left(1 \pm 6 \delta^{1 / 3}\right) ; \\
& \text { conversely, }
\end{aligned}
$$

(b) if all but $\delta\left|\mathcal{K}_{2,2}^{(2)}\left(P^{12}\right)\right|$ many elements $(\{x, y\},\{a, b\}) \in \mathcal{K}_{2,2}^{(2)}\left(P^{12}\right)$ satisfy

$$
\operatorname{deg}_{L_{x y}}(a, b)=\left(\frac{\alpha^{2}}{\ell}\right)^{2} \frac{n}{\ell^{2}}(1 \pm \delta),
$$

then $\mathcal{H}$ is $\left(\alpha, 3 \delta^{1 / 3}\right)_{2}$-minimal w.r.t. $P$. 
4.1. Proofs. In all that follows, $\mathcal{H}$ and $P$ are as in Setup 2.1. The constants $\alpha, \delta, \ell$ are fixed, and we shall take $\varepsilon=\varepsilon(\alpha, \delta, \ell)>0$ sufficiently small and $n=n(\alpha, \delta, \ell, \varepsilon)$ sufficiently large whenever needed. We denote an application of the Cauchy-Schwarz inequality by 'CS', and we denote by $o(1)$ a quantity vanishing as $n \rightarrow \infty$.

Proof of Proposition 4.1. Here, we prove only the promised lower bound for $\left|\mathcal{K}_{2,2,2}^{(3)}(\mathcal{H})\right|$. The proof of the corresponding lower bound for $\left|\mathcal{K}_{1,2,2}^{(3)}(\mathcal{H})\right|$ is similar (and can, in fact, be derived from the calculations below).

Summing over all $(\{x, y\},\{a, b\}) \in \mathcal{K}_{2,2}^{(2)}\left(P^{12}\right)$ yields

$$
\begin{aligned}
\left|\mathcal{K}_{2,2,2}^{(3)}(\mathcal{H})\right|= & \sum_{\mathcal{K}_{2,2}^{(2)}\left(P^{12}\right)}\left(\begin{array}{c}
\operatorname{deg}_{L_{x y}}(a, b) \\
2
\end{array}\right)=\left(\frac{1}{2}-o(1)\right) \sum_{\mathcal{K}_{2,2}^{(2)}\left(P^{12}\right)} \operatorname{deg}_{L_{x y}}^{2}(a, b) \\
& \stackrel{\mathrm{CS}}{\geq}\left(\frac{1}{2}-o(1)\right)\left|\mathcal{K}_{2,2}^{(2)}\left(P^{12}\right)\right|^{-1}\left(\sum_{\mathcal{K}_{2,2}^{(2)}\left(P^{12}\right)} \operatorname{deg}_{L_{x y}}(a, b)\right)^{2} \\
& =\left(\frac{1}{2}-o(1)\right)\left|\mathcal{K}_{2,2}^{(2)}\left(P^{12}\right)\right|^{-1}\left|\mathcal{K}_{2,2,1}^{(3)}(\mathcal{H})\right|^{2} \stackrel{(43)}{\geq}\left(2-\varepsilon^{1 / 3}\right) \frac{\ell^{4}}{n^{4}}\left|\mathcal{K}_{2,2,1}^{(3)}(\mathcal{H})\right|^{2},
\end{aligned}
$$

where we used $\varepsilon>0$ sufficiently small and $n$ sufficiently large. Summing over all $(\{a, b\},\{u\}) \in$ $\mathcal{K}_{2,1}^{(2)}\left(P^{23}\right)$ yields

$$
\begin{aligned}
\left|\mathcal{K}_{2,2,1}^{(3)}(\mathcal{H})\right|= & \sum_{\mathcal{K}_{2,1}^{(2)}\left(P^{23}\right)}\left(\begin{array}{c}
\operatorname{deg}_{L_{u}}(a, b) \\
2
\end{array}\right)=\left(\frac{1}{2}-o(1)\right) \sum_{\mathcal{K}_{2,1}^{(2)}\left(P^{23}\right)} \operatorname{deg}_{L_{u}}^{2}(a, b) \\
& \stackrel{\mathrm{CS}}{\geq}\left(\frac{1}{2}-o(1)\right)\left|\mathcal{K}_{2,1}^{(2)}\left(P^{23}\right)\right|^{-1}\left(\sum_{\mathcal{K}_{2,1}^{(2)}\left(P^{23}\right)} \operatorname{deg}_{L_{u}}(a, b)\right)^{2} \\
& \stackrel{(43)}{\geq}\left(1-\varepsilon^{1 / 3}\right) \frac{\ell^{2}}{n^{3}}\left(\sum_{\mathcal{K}_{2,1}^{(2)}\left(P^{23}\right)} \operatorname{deg}_{L_{u}}(a, b)\right)^{2},
\end{aligned}
$$

where we used that $\varepsilon>0$ is sufficiently small and $n$ is sufficiently large. Note that

$$
\begin{aligned}
\sum_{\mathcal{K}_{2,1}^{(2)}\left(P^{23}\right)} \operatorname{deg}_{L_{u}}(a, b) & =\sum_{\{x, u\} \in P^{13}}\left(\begin{array}{c}
\operatorname{deg}_{L_{x}}(u) \\
2
\end{array}\right)=\left(\frac{1}{2}-o(1)\right) \sum_{\{x, c\} \in P^{13}} \operatorname{deg}_{L_{x}}^{2}(u) \\
& \stackrel{\mathrm{CS}}{\geq}\left(\frac{1}{2}-o(1)\right)\left|P^{13}\right|^{-1}\left(\sum_{\{x, u\} \in P^{13}} \operatorname{deg}_{L_{x}}(u)\right)^{2}=\left(\frac{1}{2}-o(1)\right)\left|P^{13}\right|^{-1}|\mathcal{H}|^{2} .
\end{aligned}
$$

Now, the $\varepsilon$-regularity of $P^{13}$ gives $\left|P^{13}\right|=\left(n^{2} / \ell\right)(1 \pm \varepsilon)$ and Setup 2.1 and Fact 1.2 ensure

$$
|\mathcal{H}|=\alpha\left|\mathcal{K}_{3}(P)\right|=\alpha \frac{n^{3}}{\ell^{3}}\left(1 \pm \varepsilon^{1 / 3}\right)
$$


so that, in view of (46) (and $\varepsilon>0$ sufficiently small and $n$ sufficiently large),

$$
\sum_{\mathcal{K}_{2,1}^{(2)}\left(P^{23}\right)} \operatorname{deg}_{L_{u}}(a, b) \geq\left(\frac{1}{2}-\varepsilon^{1 / 4}\right) \alpha^{2} \frac{n^{4}}{\ell^{5}} .
$$

Combining (44)-(48) and employing $\varepsilon>0$ sufficiently small and $n$ sufficiently large, we conclude

$$
\left|\mathcal{K}_{2,2,2}^{(3)}(\mathcal{H})\right| \geq \frac{\alpha^{8}}{\ell^{12}}\left(\begin{array}{l}
n \\
2
\end{array}\right)^{3}\left(1-\varepsilon^{1 / 10}\right),
$$

as promised.

Proof of Proposition 4.4. Suppose $\mathcal{H}$ is $(\alpha, \delta)_{2}$-minimal w.r.t. P. We show $\mathcal{H}$ is also $(\alpha, \delta)_{1}$ minimal w.r.t. $P$. For this, note that it suffices to prove any of the inequalities in (35) since swapping the roles of $V_{1}, V_{2}, V_{3}$ preserves $(\alpha, \delta)_{2}$-minimality. We show $\left|\mathcal{K}_{2,2,1}^{(3)}(\mathcal{H})\right| \leq$ $\left(\alpha^{4} / \ell^{8}\right) n\left(\begin{array}{l}n \\ 2\end{array}\right)^{2}(1+\delta)$. Indeed, the inequalities of (44) give

$$
\left|\mathcal{K}_{2,2,1}^{(3)}(\mathcal{H})\right|^{2} \leq(2+o(1))\left|\mathcal{K}_{2,2}^{(2)}\left(P^{12}\right)\right|\left|\mathcal{K}_{2,2,2}^{(3)}(\mathcal{H})\right| \stackrel{(43)}{\leq}(2+o(1)) \frac{1}{\ell^{4}}\left(\begin{array}{l}
n \\
2
\end{array}\right)^{2}\left(1+\varepsilon^{1 / 2}\right)\left|\mathcal{K}_{2,2,2}^{(3)}(\mathcal{H})\right|
$$

Since $\mathcal{H}$ is $(\alpha, \delta)_{2}$-minimal, i.e., $\left|\mathcal{K}_{2,2,2}^{(3)}(\mathcal{H})\right| \leq\left(\alpha^{8} / \ell^{12}\right)\left(\begin{array}{l}n \\ 2\end{array}\right)^{3}(1+\delta)$, it now follows (with $\varepsilon$ sufficiently small and $n$ sufficiently large) that $\left|\mathcal{K}_{2,2,1}^{(3)}(\mathcal{H})\right| \leq\left(\alpha^{4} / \ell^{8}\right) n\left(\begin{array}{c}n \\ 2\end{array}\right)^{2}(1+\delta)$.

In our proofs below, we shall use the following well-known 'approximate' version of the Cauchy-Schwarz inequality (for a reference and a proof, see, for example, Proposition 1, p. 5, in [42]).

Fact 4.7 (Approximate Cauchy-Schwarz). Let $\gamma>0$ be given and suppose $a_{1}, \ldots, a_{s} \geq 0$ satisfy

(1) $\sum_{i=1}^{s} a_{i} \geq a s(1-\gamma)$,

(2) $\sum_{i=1}^{s} a_{i}^{2}<a^{2} s(1+\gamma)$.

Then $\left|\left\{i \in[s]:\left|a-a_{i}\right|<2 \gamma^{1 / 3} a\right\}\right|>\left(1-2 \gamma^{1 / 3}\right) s$.

Proof of Proposition 4.5. We sketch the proof of Statement (1) (for single links) (the proof of Statement (2) for colinks $L_{x y}$ is similar). Assume that $\mathcal{H}$ is $(\alpha, \delta)_{1}$-minimal w.r.t. $P$. We show that all but $3 \delta^{1 / 3} n$ vertices $x \in V_{1}$ satisfy $\left|L_{x}\right|=\left(\alpha / \ell^{3}\right) n^{2}\left(1 \pm 3 \delta^{1 / 3}\right)$. In our proof, we shall need the following inequalities, which are virtually identical to (45) and (46):

$$
\begin{aligned}
\left|\mathcal{K}_{1,2,2}^{(3)}(\mathcal{H})\right|= & \sum_{\mathcal{K}_{1,2}^{(2)}\left(P^{12}\right)}\left(\begin{array}{c}
\operatorname{deg}_{L_{x}}(a, b) \\
2
\end{array}\right) \\
& \stackrel{\mathrm{CS}}{\geq}\left(\frac{1}{2}-o(1)\right)\left|\mathcal{K}_{1,2}^{(2)}\left(P^{12}\right)\right|^{-1}\left(\sum_{\mathcal{K}_{1,2}^{(2)}\left(P^{12}\right)} \operatorname{deg}_{L_{x}}(a, b)\right)^{2}
\end{aligned}
$$

(where we sum over all $(\{x\},\{a, b\}) \in \mathcal{K}_{1,2}^{(2)}\left(P^{12}\right)$ ) and

$$
\sum_{\mathcal{K}_{1,2}^{(2)}\left(P^{12}\right)} \operatorname{deg}_{L_{x}}(a, b)=\sum_{\{x, u\} \in P^{13}}\left(\begin{array}{c}
\operatorname{deg}_{L_{x}}(u) \\
2
\end{array}\right)=\left(\frac{1}{2}-o(1)\right) \sum_{\{x, u\} \in P^{13}} \operatorname{deg}_{L_{x}}^{2}(u) .
$$


We want to use Fact 4.7 (with the $a_{i}$ 's being the terms $\left|L_{x}\right|, x \in V_{1}$ ). To that end, we need the following preparations. Observe that

$$
\sum_{x \in V_{1}}\left|L_{x}\right|=|\mathcal{H}| \stackrel{(47)}{\geq} \alpha \frac{n^{3}}{\ell^{3}}\left(1-\varepsilon^{1 / 3}\right) \geq\left(\alpha \frac{n^{2}}{\ell^{3}}\right) n(1-3 \delta) .
$$

To bound $\sum_{x \in V_{1}}\left|L_{x}\right|^{2}$, we separate the sum into two groups. Let $V_{1}^{\prime}$ denote those vertices $x \in V_{1}$ for which $\operatorname{deg}_{P^{13}}(x)=\left(\ell^{-1} \pm \varepsilon\right) n$. Then $\left|V_{1} \backslash V_{1}^{\prime}\right|<2 \varepsilon n$ and so

$$
\sum_{x \in V_{1}}\left|L_{x}\right|^{2}=\sum_{x \in V_{1}^{\prime}}\left|L_{x}\right|^{2}+\sum_{x \in V_{1} \backslash V_{1}^{\prime}}\left|L_{x}\right|^{2} \leq 2 \varepsilon n^{5}+\sum_{x \in V_{1}^{\prime}}\left|L_{x}\right|^{2} .
$$

We now bound $\sum_{x \in V_{1}^{\prime}}\left|L_{x}\right|^{2}$. To that end, observe that

$$
\begin{aligned}
& \sum_{\{x, u\} \in P^{13}} \operatorname{deg}_{L_{x}}^{2}(u)=\sum_{x \in V_{1}} \sum_{u \in N_{P^{13}}(x)} \operatorname{deg}_{L_{x}}^{2}(u) \geq \sum_{x \in V_{1}^{\prime}} \sum_{u \in N_{P^{13}}(x)} \operatorname{deg}_{L_{x}}^{2}(u) \\
& \stackrel{\mathrm{CS}}{\geq} \sum_{x \in V_{1}^{\prime}} \operatorname{deg}_{P^{13}}^{-1}(x)\left(\sum_{u \in N_{P_{13}}(x)} \operatorname{deg}_{L_{x}}(u)\right)^{2}=\sum_{x \in V_{1}^{\prime}} \operatorname{deg}_{P^{13}}^{-1}(x)\left|L_{x}\right|^{2} \geq \frac{\ell}{n}(1+\ell \varepsilon)^{-1} \sum_{x \in V_{1}^{\prime}}\left|L_{x}\right|^{2},
\end{aligned}
$$

where we used that each $x \in V_{1}^{\prime}$ satisfies $\operatorname{deg}_{P^{13}}(x)=\left(\ell^{-1} \pm \varepsilon\right) n$. These inequalities, combined with (50) and (51) (and $\varepsilon>0$ sufficiently small and $n$ sufficiently large) then yield

$$
\left(\sum_{x \in V_{1}^{\prime}}\left|L_{x}\right|^{2}\right)^{2} \leq\left(8+\varepsilon^{1 / 2}\right) \frac{n^{2}}{\ell^{2}}\left|\mathcal{K}_{1,2}^{(2)}\left(P^{12}\right)\right|\left|\mathcal{K}_{1,2,2}^{(3)}(\mathcal{H})\right| \stackrel{(43)}{\leq}\left(4+\varepsilon^{1 / 3}\right) \frac{n^{5}}{\ell^{4}}\left|\mathcal{K}_{1,2,2}^{(3)}(\mathcal{H})\right|
$$

Since $\mathcal{H}$ is $(\alpha, \delta)_{1}$-minimal w.r.t. $P$, i.e., $\left|\mathcal{K}_{1,2,2}^{(3)}(\mathcal{H})\right| \leq\left(\alpha^{4} / \ell^{8}\right) n\left(\begin{array}{c}n \\ 2\end{array}\right)^{2}(1+\delta)$, we then see (with $0<\varepsilon \ll \delta)$

$$
\sum_{x \in V_{1}^{\prime}}\left|L_{x}\right|^{2} \leq\left(\alpha \frac{n^{2}}{\ell^{3}}\right)^{2} n(1+2 \delta) .
$$

Combining (52) and (53), we then see (with $0<\varepsilon \ll \delta$ )

$$
\sum_{x \in V_{1}}\left|L_{x}\right|^{2} \leq 2 \varepsilon n^{5}+\left(\alpha \frac{n^{2}}{\ell^{3}}\right)^{2} n(1+2 \delta) \leq\left(\alpha \frac{n^{2}}{\ell^{3}}\right)^{2} n(1+3 \delta) .
$$

Now, to conclude the proof of (Statement (1) of) Proposition 4.5, we set, as in Fact 4.7,

$$
\gamma=3 \delta, \quad s=n, \quad a=\alpha \frac{n^{2}}{\ell^{3}}
$$

and set the $a_{i}$ 's to be the terms $\left|L_{x}\right|, x \in V_{1}$. Fact 4.7 applies to say that for all but $2(3 \delta)^{1 / 3} n$ many vertices $x \in V_{1}$, we have

$$
\left|L_{x}\right|=\alpha \frac{n^{2}}{\ell^{3}}\left(1 \pm 2(3 \delta)^{1 / 3}\right) .
$$

Since $2 \cdot 3^{1 / 3}<3$, we have shown that all but $3 \delta^{1 / 3} n$ vertices $x \in V_{1}$ have $\left|L_{x}\right|=\alpha\left(n^{2} / \ell^{3}\right)(1 \pm$ $3 \delta^{1 / 3}$ ), as promised by Statement (1) of Proposition 4.5. 
Proof of Proposition 4.6. We sketch the proof of Statement (2) (involving colinks $L_{x y}$ ) (the proof of Statement (1) (involving links) is similar). To that end, we shall concentrate on the proof of Statement (2)-(a), since the proof of Statement (2)-(b) is quite standard. (Indeed, to briefly sketch it, suppose all but $\delta\left|\mathcal{K}_{2,2}^{(2)}\left(P^{12}\right)\right|$ many copies $(\{x, y\},\{a, b\})$ of $C_{4}$ in $P^{12}$ satisfy $\operatorname{deg}_{L_{x y}}(a, b)=\left(\alpha^{2} / \ell\right)^{2}\left(n / \ell^{2}\right)(1 \pm \delta)$. Recall (cf. (44))

$$
\left|\mathcal{K}_{2,2,2}^{(3)}(\mathcal{H})\right|=\sum_{\mathcal{K}_{2,2}^{(2)}\left(P^{12}\right)}\left(\begin{array}{c}
\operatorname{deg}_{L_{x y}}(a, b) \\
2
\end{array}\right)
$$

where our assumption is that we may control (from above) all but a $\delta$ proportion of the terms in the sum. Therefore, to establish an upper bound on $\left|\mathcal{K}_{2,2,2}^{(3)}(\mathcal{H})\right|$, we need to estimate the contribution of those at most $\delta$ proportion of terms over which we no not have such tight control. To this end, we use the $\varepsilon$-regularity of the graphs $P^{12}, P^{23}, P^{13}$ to conclude that all but $\varepsilon^{1 / 3} n^{4}$ many terms $(\{x, y\},\{a, b\})$ satisfy $\operatorname{deg}_{L_{x y}}(a, b) \leq 2 n / \ell^{4}$, which essentially ends the argument.)

We now prove Statement $(2)-(\mathrm{a})$. To that end, assume $\mathcal{H}$ is $(\alpha, \delta)_{2}$-minimal w.r.t. $P$. We prove that for all but $3 \delta^{1 / 3}\left|\mathcal{K}_{2,2}^{(2)}\left(P^{12}\right)\right|$ many elements $(\{x, y\},\{a, b\}) \in \mathcal{K}_{2,2}^{(2)}\left(P^{12}\right)$, we have

$$
\operatorname{deg}_{L_{x y}}(a, b)=\left(\frac{\alpha^{2}}{\ell}\right) \frac{n}{\ell^{2}}\left(1 \pm 3 \delta^{1 / 3}\right)
$$

We want to use Fact 4.7 (with the $a_{i}$ 's being the terms $\operatorname{deg}_{L_{x y}}(a, b),(\{x, y\},\{a, b\}) \in$ $\left.\mathcal{K}_{2,2}^{(2)}\left(P^{12}\right)\right)$. To that end, we need the following preparations. From (44), we infer

$$
\sum_{\mathcal{K}_{2,2}^{(2)}\left(P^{12}\right)} \operatorname{deg}_{L_{x y}}^{2}(a, b) \leq(2+o(1))\left|\mathcal{K}_{2,2,2}^{(3)}(\mathcal{H})\right|
$$

so that, by the $(\alpha, \delta)_{2}$-minimality of $\mathcal{H}$ (and $n$ sufficiently large), we have

$$
\sum_{\mathcal{K}_{2,2}^{(2)}\left(P^{12}\right)} \operatorname{deg}_{L_{x y}}^{2}(a, b) \leq \alpha^{8} \frac{n^{6}}{4 \ell^{12}}(1+2 \delta) \stackrel{(43)}{\leq}\left[\left(\frac{\alpha^{2}}{\ell}\right)^{2} \frac{n}{\ell^{2}}\right]^{2}\left|\mathcal{K}_{2,2}^{(2)}\left(P^{12}\right)\right|(1+3 \delta),
$$

where in the last inequality we used $\varepsilon>0$ sufficiently small. On the other hand,

$$
\begin{aligned}
\left(\sum_{\mathcal{K}_{2,2}^{(2)}\left(P^{12}\right)} \operatorname{deg}_{L_{x y}}(a, b)\right)^{2} & =\left|\mathcal{K}_{2,2,1}^{(3)}(\mathcal{H})\right|^{2} \stackrel{(45)}{\geq}\left[\left(1-\varepsilon^{1 / 3}\right) \frac{\ell^{2}}{n^{3}}\left(\sum_{\mathcal{K}_{2,1}^{(2)}\left(P^{23}\right)} \operatorname{deg}_{L_{u}}(a, b)\right)^{2}\right]^{2} \\
& \stackrel{(48)}{\geq}\left[\left(1-\varepsilon^{1 / 3}\right) \frac{\ell^{2}}{n^{3}}\left(\left(\frac{1}{2}-\varepsilon^{1 / 4}\right) \alpha^{2} \frac{n^{4}}{\ell^{5}}\right)^{2}\right]^{2} \geq \alpha^{8} \frac{n^{10}}{16 \ell^{16}}\left(1-\varepsilon^{1 / 5}\right)
\end{aligned}
$$


(using $\varepsilon>0$ sufficiently small), so that

$$
\begin{aligned}
\sum_{\mathcal{K}_{2,2}^{(2)}\left(P^{12}\right)} \operatorname{deg}_{L_{x y}}(a, b) \geq\left[\left(\frac{\alpha^{2}}{\ell}\right)^{2} \frac{n}{\ell^{2}}\right] \frac{n^{4}}{4 \ell^{4}}\left(1-\varepsilon^{1 / 5}\right) \stackrel{(43)}{\geq}\left[\left(\frac{\alpha^{2}}{\ell}\right)^{2} \frac{n}{\ell^{2}}\right]\left|\mathcal{K}_{2,2}^{(2)}\left(P^{12}\right)\right|\left(1-\varepsilon^{1 / 6}\right) \\
\geq\left[\left(\frac{\alpha^{2}}{\ell}\right)^{2} \frac{n}{\ell^{2}}\right]\left|\mathcal{K}_{2,2}^{(2)}\left(P^{12}\right)\right|(1-3 \delta) .
\end{aligned}
$$

Now to conclude the proof of (Statement (2)-(a) of) Proposition 4.6, we set, as in Fact 4.7,

$$
\gamma=3 \delta, \quad s=\left|\mathcal{K}_{2,2}^{(2)}\left(P^{12}\right)\right|, \quad a=\left(\frac{\alpha^{2}}{\ell}\right)^{2} \frac{n}{\ell^{2}}
$$

and set the $a_{i}$ 's to be the terms $\operatorname{deg}_{L_{x y}}(a, b),(\{x, y\},\{a, b\}) \in \mathcal{K}_{2,2}^{(2)}\left(P^{12}\right)$. Fact 4.7 applies to say that for all but $2(3 \delta)^{1 / 3}\left|\mathcal{K}_{2,2}^{(2)}\left(P^{12}\right)\right|$ many terms, we have

$$
\operatorname{deg}_{L_{x y}}(a, b)=\left(\frac{\alpha^{2}}{\ell}\right)^{2} \frac{n}{\ell^{2}}\left(1 \pm 2(3 \delta)^{1 / 3}\right) .
$$

Since $2 \cdot 3^{1 / 3}<3$, this proves Statement (2)-(a) of Proposition 4.6.

\section{Proof of Counting Lemma}

In this section, we prove the Counting Lemma, Theorem 2.7. Our proof proceeds formally by induction on $k \geq 3$.

Base Case. The base case $k=3$ holds trivially. Indeed, $\mathcal{H}^{123}=\mathcal{H}$ has density $\alpha=$ $|\mathcal{H}| /\left|\mathcal{K}_{3}^{(2)}(P)\right|$ with respect to $P=P^{12} \cup P^{23} \cup P^{13}$. With constant $\varepsilon \leq \delta$ sufficiently small with respect to $\ell^{-1}$, Fact 1.2 renders

$$
\left|\mathcal{K}_{3}^{(2)}(P)\right|=\frac{n^{3}}{\ell^{3}}\left(1 \pm \varepsilon^{1 / 3}\right) \quad \Longrightarrow \quad\left|\mathcal{K}_{3}^{(3)}(\mathcal{H})\right|=|\mathcal{H}|=\alpha \frac{n^{3}}{\ell^{3}}\left(1 \pm \varepsilon^{1 / 3}\right)=\alpha \frac{n^{3}}{\ell^{3}}\left(1 \pm \delta^{1 / 360}\right)
$$

confirming the Counting Lemma for $k=3$.

Induction Step. We assume Theorem 2.7 holds up through $k-1 \geq 3$ and consider Theorem 2.7 for $k \geq 4$. With appropriately defined constants (which we comment on momentarily), we consider $\mathcal{H}=\bigcup_{1 \leq h<i<j \leq k} \mathcal{H}^{\text {hij }}$ and $P=\bigcup_{1 \leq i<j \leq k} P^{i j}$ as in Setup 2.6 on $k$-partition $V=$ $V_{1} \cup \ldots \cup V_{k},\left|V_{1}\right|=\ldots=\left|V_{k}\right|=n$, where each $\mathcal{H}^{h i j}, 1 \leq h<i<j \leq k$, is $(\alpha, \delta)$-minimal with respect to $P^{h i} \cup P^{i j} \cup P^{h j}$ and each $P^{i j}, 1 \leq i<j \leq k$, is $\left(\ell^{-1}, \varepsilon\right)$-regular. We prove

$$
\left|\mathcal{K}_{k}^{(3)}(\mathcal{H})\right|=\frac{\alpha^{\left(\begin{array}{c}
k \\
3
\end{array}\right)}}{\ell^{\left(\begin{array}{c}
k \\
2
\end{array}\right)}} n^{k}\left(1 \pm \delta^{\frac{1}{120 k}}\right) .
$$

We now discuss the constants $k, \alpha, \delta_{0}, \delta, \ell, \varepsilon, n$ required to enable (54).

With $k$ fixed (by Induction), the Counting Lemma is quantified as $\forall \alpha, \exists \delta_{0}: \forall \delta<\delta_{0}, \forall \ell, \exists \varepsilon$ : with $n$ sufficiently large... 
This quantification is consistent with the following hierarchy:

$$
\frac{1}{k}, \alpha \gg \delta_{0}>\delta \geq \min \left\{\delta, \frac{1}{\ell}\right\} \gg \varepsilon \gg \frac{1}{n} .
$$

Rather than presenting a tedious determination of the constants $\alpha, \delta_{0}, \delta, \ell, \varepsilon, n$, we shall, in all calculations below, appeal to the hierarchy in (55).

To establish (54), we find it convenient to reformulate the Counting Lemma in terms of a slightly different language given by auxiliary bipartite graphs $\Lambda \subseteq \Pi$, which we now define.

Construction 5.1 (defining $\Lambda \subseteq \Pi$ ). With k-partition $V_{1} \cup \ldots \cup V_{k}$ of $\mathcal{H}=\bigcup_{1 \leq h<i<j \leq k} \mathcal{H}^{h i j}$ and $P=\bigcup_{1<i<j<k} P^{i j}$ given as in Setup 2.6, define auxiliary bipartite graphs $\Lambda \subseteq \Pi$ with bipartition $X \cup Y$ as follows:

$$
X=V_{1} \quad \text { and } \quad Y=\mathcal{K}_{k-1}^{(2)}\left(P\left[V_{2}, \ldots, V_{k}\right]\right)=\mathcal{K}_{k-1}^{(2)}\left(\bigcup_{1<i<j \leq k} P^{i j}\right)
$$

where elements of $Y$, each denoted by $K^{-}$, correspond to the vertex sets of graph $(k-1)$-cliques $K_{k-1}^{(2)}$ in the $(k-1)$-partite graph $P\left[V_{2}, \ldots, V_{k}\right]=\bigcup_{1<i<j \leq k} P^{i j}$. Note that

$$
|X|=n \quad \text { and } \quad|Y| \stackrel{\text { Fact } 1.2}{=} \frac{n^{k-1}}{\ell^{\left(\begin{array}{c}
k-1 \\
2
\end{array}\right)}}\left(1 \pm \varepsilon^{\frac{1}{k-1}}\right) .
$$

- For $x \in X$ and $K^{-} \in Y$,

$\left\{x, K^{-}\right\} \in \Pi \Longleftrightarrow\{x\} \cup K^{-} \in \mathcal{K}_{k}^{(2)}(P) \Longleftrightarrow K^{-} \in \mathcal{K}_{k-1}^{(2)}\left(P_{x}\right) \Longleftrightarrow K^{-} \subset N_{P}(x)$

where $P_{x}=P\left[N_{P}(x)\right]=P\left[N_{P^{12}}(x), \ldots, N_{P^{1 k}}(x)\right]$ is the subgraph of $P$ induced on the neighborhood $N_{P}(x)=N_{P^{12}}(x) \cup \cdots \cup N_{P^{1 k}}(x)$ of $x$.

- For $x \in X$ and $K^{-} \in Y$,

$$
\left\{x, K^{-}\right\} \in \Lambda \Longleftrightarrow K^{-} \in \mathcal{K}_{k-1}^{(2)}\left(L_{x}\right) \Longleftrightarrow\left(\begin{array}{c}
K^{-} \\
2
\end{array}\right) \subset L_{x}
$$

where $L_{x}=\left\{\{y, z\} \in P_{x}:\{x, y, z\} \in \mathcal{H}\right\} \subseteq P_{x}$ is the link graph of $x$ (cf. (36)).

Note that since each $x \in V_{1}$ has $L_{x} \subseteq P_{x}$, it follows that $\Lambda \subseteq \Pi$. We also note the following useful but standard fact concerning graph $\Pi$.

Fact 5.2. With $\varepsilon$ given in (55), the graph $\Pi$ is $\left(\ell^{1-k}, \varepsilon^{1 / 3}\right)$-regular.

We omit the standard proof of Fact 5.2.

We now make a few straightforward observations establishing connections between graphs $\Lambda$ and $\Pi$ and the Counting Lemma, Theorem 2.7. For the purpose of stating these observations, set

$$
Y_{\mathcal{H}} \stackrel{\text { def }}{=} \mathcal{K}_{k-1}^{(3)}\left(\mathcal{H}\left[V_{2}, \ldots, V_{k}\right]\right)=\mathcal{K}_{k-1}^{(3)}\left(\bigcup_{1<h<i<j \leq k} \mathcal{H}^{h i j}\right)
$$

and note that elements of $Y_{\mathcal{H}}$ correspond to vertex sets of hypergraph $(k-1)$-cliques $K_{k-1}^{(3)}$ in the $(k-1)$-partite 3 -graph $\mathcal{H}\left[V_{2}, \ldots, V_{k}\right]=\bigcup_{1<h<i<j \leq k} \mathcal{H}^{h i j}$. We observe the following:

\section{Observations.}

(1) Since $\mathcal{H} \subseteq \mathcal{K}_{3}^{(2)}(P)$ (cf. Setup 2.6), $Y_{\mathcal{H}} \subseteq Y$ is a subset of the vertices $Y$. 
(2) By our Induction Hypothesis on the Counting Lemma (for $k-1)$,

$$
\begin{gathered}
\left|Y_{\mathcal{H}}\right|=\left|\mathcal{K}_{k-1}^{(3)}\left(\mathcal{H}\left[V_{2}, \ldots, V_{k}\right]\right)\right|=\left|\mathcal{K}_{k-1}^{(3)}\left(\bigcup_{1<h<i<j \leq k} \mathcal{H}^{h i j}\right)\right| \\
=\frac{\alpha^{\left(\begin{array}{c}
k-1 \\
3
\end{array}\right)}}{\ell^{\left(\begin{array}{c}
k-1 \\
2
\end{array}\right)}} n^{k-1}\left(1 \pm \delta^{\frac{1}{120(k-1)}}\right) \stackrel{(55),(56)}{=} \alpha^{\left(\begin{array}{c}
k-1 \\
3
\end{array}\right)}|Y|\left(1 \pm 2 \delta^{\frac{1}{120(k-1)}}\right) .
\end{gathered}
$$

$$
\left|\mathcal{K}_{k}^{(3)}(\mathcal{H})\right|=\sum_{K^{-} \in Y_{\mathcal{H}}} \operatorname{deg}_{\Lambda}\left(K^{-}\right)
$$

We see from (57) and (58) that, to prove the Counting Lemma, it suffices to analyze the terms in the sum (58). Proposition 5.3 does precisely this.

Proposition 5.3 (key to Counting Lemma). All but $3 \delta^{\frac{1}{111(k-1)}}|Y|$ vertices $K^{-} \in Y$ satisfy

$$
\operatorname{deg}_{\Lambda}\left(K^{-}\right)=\frac{\alpha^{\left(\begin{array}{c}
k-1 \\
2
\end{array}\right)}}{\ell^{k-1}}|X|\left(1 \pm 3 \delta^{\frac{1}{111(k-1)}}\right)=\frac{\alpha^{\left(\begin{array}{c}
k-1 \\
2
\end{array}\right)}}{\ell^{k-1}} n\left(1 \pm 3 \delta^{\frac{1}{111(k-1)}}\right) .
$$

We defer the proof of Proposition 5.3 to Section 5.2. We proceed now with the confirmation that the Counting Lemma follows from Proposition 5.3.

5.1. Proposition 5.3 $\Longrightarrow$ Counting Lemma. Combining Proposition 5.3 with (57) and (58), the proof of the Counting Lemma is virtually immediate. Indeed, Proposition 5.3 and (57) imply 'almost all' (cf. $\delta \ll \alpha$ in (55)) of the (57) many terms in (58) have degree in (59). As such,

$$
\left|\mathcal{K}_{k}^{(3)}(\mathcal{H})\right|=\sum_{K^{-} \in Y_{\mathcal{H}}} \operatorname{deg}_{\Lambda}\left(K^{-}\right) \sim \frac{\alpha^{\left(\begin{array}{c}
k-1 \\
2
\end{array}\right)}}{\ell^{k-1}} n \times \frac{\alpha^{\left(\begin{array}{c}
k-1 \\
3
\end{array}\right)}}{\ell^{\left(\begin{array}{c}
k-1 \\
2
\end{array}\right)}} n^{k-1}=\frac{\alpha^{\left(\begin{array}{c}
k \\
3
\end{array}\right)}}{\ell^{\left(\begin{array}{c}
k \\
2
\end{array}\right)}} n^{k}
$$

where $\sim$ denotes an 'essential' equality we make precise by accounting for the degrees of vertices $K^{-} \in Y_{\mathcal{H}}$ not satisfying (59).

To make (60) precise, we will use (59) for all $K^{-} \in Y_{\mathcal{H}}$ satisfying it, and for the remaining ones, we employ the natural upper bound $\operatorname{deg}_{\Lambda}\left(K^{-}\right) \leq \operatorname{deg}_{\Pi}\left(K^{-}\right)$(recall $\Lambda \subseteq \Pi$ ). As such, Fact 5.2 implies

$$
\text { all but } 2 \varepsilon^{1 / 3}|Y| \text { vertices } K^{-} \in Y \text { satisfy }
$$

Now, set

(62) $Y_{\Lambda}=\left\{K^{-} \in Y: K^{-}\right.$satisfies (59) $\} \quad$ and $Y_{\Pi}=\left\{K^{-} \in Y: K^{-}\right.$satisfies (61) $\}$

so that

$$
\begin{gathered}
\left|Y_{\mathcal{H}} \backslash Y_{\Lambda}\right| \leq\left|Y \backslash Y_{\Lambda}\right| \stackrel{\text { Prop. }}{\leq} 3.3 \frac{1}{\leq} 3 \delta^{\frac{1}{111(k-1)}}|Y| \stackrel{(56)}{\leq} 6 \delta^{\frac{1}{111(k-1)}} \frac{n^{k-1}}{\ell^{\left(\begin{array}{c}
k-1 \\
2
\end{array}\right)}} \\
\left|Y_{\mathcal{H}} \backslash Y_{\Pi}\right| \leq\left|Y \backslash Y_{\Pi}\right| \stackrel{(61)}{\leq} 2 \varepsilon^{1 / 3}|Y| \stackrel{(56)}{\leq} 4 \varepsilon^{1 / 3} \frac{n^{k-1}}{\ell^{\left(\begin{array}{c}
k-1 \\
2
\end{array}\right)}}
\end{gathered}
$$


We now make (60) precise. Observe

$$
\left|\mathcal{K}_{k}^{(3)}(\mathcal{H})\right|=\sum_{K^{-} \in Y_{\Lambda} \cap Y_{\mathcal{H}}} \operatorname{deg}_{\Lambda}\left(K^{-}\right)+\sum_{K^{-} \in\left(Y_{\Pi} \backslash Y_{\Lambda}\right) \cap Y_{\mathcal{H}}} \operatorname{deg}_{\Lambda}\left(K^{-}\right)+\sum_{K^{-} \in Y_{\mathcal{H}} \backslash\left(Y_{\Lambda} \cup Y_{\Pi}\right)} \operatorname{deg}_{\Lambda}\left(K^{-}\right) .
$$

To obtain the formula for $\left|\mathcal{K}_{k}^{(3)}(\mathcal{H})\right|$ promised in (54), we need to bound (65) from above and below. We do so now.

To see the lower bound, we use that every $K^{-} \in Y_{\Lambda} \cap Y_{\mathcal{H}}$ satisfies $\operatorname{deg}_{\Lambda}\left(K^{-}\right) \geq\left(\alpha^{\left.\left(\begin{array}{c}k-1 \\ 2\end{array}\right) / \ell^{k-1}\right) n(1-}\right.$ $3 \delta^{111(k-1)}$ ) (cf. (59)) to obtain (from (65))

$$
\begin{gathered}
\left|\mathcal{K}_{k}^{(3)}(\mathcal{H})\right| \geq \frac{\alpha^{\left(\begin{array}{c}
k-1 \\
2
\end{array}\right)}}{\ell^{k-1}} n\left(1-3 \delta^{\frac{1}{111(k-1)}}\right)\left|Y_{\Lambda} \cap Y_{\mathcal{H}}\right|=\frac{\alpha^{\left(\begin{array}{c}
k-1 \\
2
\end{array}\right)}}{\ell^{k-1}} n\left(1-3 \delta^{\frac{1}{111(k-1)}}\right)\left(\left|Y_{\mathcal{H}}\right|-\left|Y_{\mathcal{H}} \backslash Y_{\Lambda}\right|\right) \\
\stackrel{(57),(63)}{\geq} \frac{\alpha^{\left(\begin{array}{c}
k \\
3
\end{array}\right)}}{\ell^{\left(\begin{array}{c}
k \\
2
\end{array}\right)}} n^{k}\left(1-3 \delta^{\frac{1}{111(k-1)}}\right)\left[1-\delta^{\frac{1}{120(k-1)}}-6 \frac{\delta^{\frac{1}{111(k-1)}}}{\alpha^{\left(\begin{array}{c}
k-1 \\
3
\end{array}\right)}}\right] \stackrel{(55)}{\geq} \frac{\alpha^{\left(\begin{array}{c}
k \\
3
\end{array}\right)}}{\ell^{\left(\begin{array}{c}
k \\
2
\end{array}\right)}} n^{k}\left(1-\delta^{\frac{1}{120 k}}\right) .
\end{gathered}
$$

To see the upper bound, we argue similarly, this time showing that the terms of (65) outside of $\sum_{K^{-} \in Y_{\Lambda} \cap Y_{\mathcal{H}}} \operatorname{deg}_{\Lambda}\left(K^{-}\right)$contribute to only little error. Indeed,

$$
\begin{aligned}
& \left|\mathcal{K}_{k}^{(3)}(\mathcal{H})\right| \leq \sum_{K^{-} \in Y_{\Lambda} \cap Y_{\mathcal{H}}} \operatorname{deg}_{\Lambda}\left(K^{-}\right)+\sum_{K^{-} \in\left(Y_{\Pi} \backslash Y_{\Lambda}\right) \cap Y_{\mathcal{H}}} \operatorname{deg}_{\Pi}\left(K^{-}\right)+n\left|Y_{\mathcal{H}} \backslash\left(Y_{\Lambda} \cup Y_{\Pi}\right)\right| \\
& \stackrel{(59),(61)}{\leq} \frac{\alpha^{\left(\begin{array}{c}
k-1 \\
2
\end{array}\right)}}{\ell^{k-1}} n\left(1+3 \delta^{\frac{1}{111(k-1)}}\right)\left|Y_{\Lambda} \cap Y_{\mathcal{H}}\right|+2 \frac{n}{\ell^{k-1}}\left|\left(Y_{\Pi} \backslash Y_{\Lambda}\right) \cap Y_{\mathcal{H}}\right|+n\left|Y_{\mathcal{H}} \backslash\left(Y_{\Lambda} \cup Y_{\Pi}\right)\right| \\
& \leq \frac{\alpha^{\left(\begin{array}{c}
k-1 \\
2
\end{array}\right)}}{\ell^{k-1}} n\left(1+3 \delta^{\frac{1}{111(k-1)}}\right)\left|Y_{\mathcal{H}}\right|+2 \frac{n}{\ell^{k-1}}\left|Y_{\mathcal{H}} \backslash Y_{\Lambda}\right|+n\left|Y_{\mathcal{H}} \backslash Y_{\Pi}\right| \\
& \stackrel{(57),(63),(64)}{\leq} \frac{\alpha^{\left(\begin{array}{c}
k \\
3
\end{array}\right)}}{\ell^{\left(\begin{array}{c}
k \\
2
\end{array}\right)}} n^{k}\left(1+3 \delta^{\frac{1}{111(k-1)}}\right)\left(1+\delta^{\frac{1}{120(k-1)}}\right)+6 \delta^{\frac{1}{111(k-1)}} \frac{n^{k}}{\ell^{\left(\begin{array}{c}
k \\
2
\end{array}\right)}}+8 \varepsilon^{1 / 3} \frac{n^{k}}{\ell^{\left(\begin{array}{c}
k-1 \\
2
\end{array}\right)}} \\
& =\frac{\alpha^{\left(\begin{array}{c}
k \\
3
\end{array}\right)}}{\ell^{\left(\begin{array}{c}
k \\
2
\end{array}\right)}} n^{k}\left[\left(1+3 \delta^{\frac{1}{111(k-1)}}\right)\left(1+\delta^{\frac{1}{120(k-1)}}\right)+6 \frac{\delta^{\frac{1}{111(k-1)}}}{\alpha^{\left(\begin{array}{c}
k \\
3
\end{array}\right)}}+8 \frac{\varepsilon^{1 / 3} \ell^{k-1}}{\alpha^{\left(\begin{array}{c}
k \\
3
\end{array}\right)}}\right] \stackrel{\left(\begin{array}{c}
55 \\
\leq
\end{array}\right.}{\leq} \frac{\alpha^{\left(\begin{array}{c}
k \\
3
\end{array}\right)}}{\ell^{\left(\begin{array}{c}
k \\
2
\end{array}\right)}} n^{k}\left(1+\delta^{\frac{1}{120 k}}\right) .
\end{aligned}
$$

This completes the proof of the induction step for the Counting Lemma.

5.2. Proof of Proposition 5.3. It remains to prove Proposition 5.3. We do so by proving the following stronger version of Proposition 5.3.

\section{Proposition 5.3*.}

- All but $\delta^{1 / 36}|X|=\delta^{1 / 36} n$ vertices $x \in X=V_{1}$ satisfy

$$
\operatorname{deg}_{\Lambda}(x)=\left(\frac{\alpha}{\ell}\right)^{\left(\begin{array}{c}
k-1 \\
2
\end{array}\right)}\left(\frac{n}{\ell}\right)^{k-1}\left(1 \pm \delta^{\frac{1}{36(k-1)}}\right) \stackrel{(56)}{=} \frac{\alpha^{\left(\begin{array}{c}
k-1 \\
2
\end{array}\right)}}{\ell^{k-1}}|Y|\left(1 \pm 2 \delta^{\frac{1}{36(k-1)}}\right) .
$$

In particular,

$$
\sum_{K^{-} \in Y} \operatorname{deg}_{\Lambda}\left(K^{-}\right) \geq\left(\frac{\alpha^{\left(\begin{array}{c}
k-1 \\
2
\end{array}\right)}}{\ell^{k-1}}|X|\right)|Y|\left(1-\delta^{\frac{1}{37(k-1)}}\right) .
$$


- all but $\delta^{1 / 36}|X|^{2}=\delta^{1 / 36} n^{2}$ pairs $x, y \in X=V_{1}$ satisfy

$$
\operatorname{deg}_{\Lambda}(x, y)=\left(\frac{\alpha^{2}}{\ell}\right)^{\left(\begin{array}{c}
k-1 \\
2
\end{array}\right)}\left(\frac{n}{\ell^{2}}\right)^{k-1}\left(1 \pm \delta^{\frac{1}{36(k-1)}}\right) \stackrel{(56)}{=}\left(\frac{\left.\alpha^{(k-1} 2\right)}{\ell^{k-1}}\right)^{2}|Y|\left(1 \pm 2 \delta^{\frac{1}{36(k-1)}}\right) .
$$

In particular,

$$
\sum_{K^{-} \in Y} \operatorname{deg}_{\Lambda}\left(K^{-}\right)^{2}<\left(\frac{\alpha^{\left(\frac{k-1}{2}\right)}}{\ell^{k-1}}|X|\right)^{2}|Y|\left(1+3 \delta^{\frac{1}{37(k-1)}}\right) .
$$

It is easy to prove Proposition 5.3 from (67) and (69) of Proposition 5.3*. We do so using the

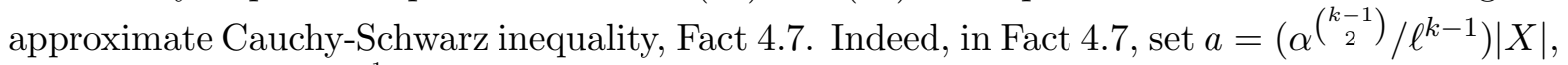
$r=|Y|$ and $\gamma=3 \delta^{\frac{1}{37(k-1)}}$. The terms $a_{i}, 1 \leq i \leq r$, correspond to terms $\operatorname{deg}_{\Lambda}\left(K^{-}\right), K^{-} \in Y$. Fact 4.7 then gives that all but $2\left(3 \delta^{\frac{1}{37(k-1)}}\right)^{1 / 3}|Y|<3 \delta^{\frac{1}{111(k-1)}}|Y|$ terms $\operatorname{deg}_{\Lambda}\left(K^{-}\right), K^{-} \in Y$, satisfy

$$
\operatorname{deg}_{\Lambda}\left(K^{-}\right)=\frac{\alpha^{\left(\begin{array}{c}
k-1 \\
2
\end{array}\right)}}{\ell^{k-1}}|X|\left(1 \pm 2\left(3 \delta^{\frac{1}{37(k-1)}}\right)^{1 / 3}\right)=\frac{\alpha^{\left(\begin{array}{c}
k-1 \\
2
\end{array}\right)}}{\ell^{k-1}}|X|\left(1 \pm 3 \delta^{\frac{1}{111(k-1)}}\right)
$$

where we used that $2 \cdot 3^{1 / 3}<3$. This proves Proposition $5.3^{*}$.

To prove Proposition 5.3*, we must show 4 things: the assertions of (66) and (68) and the implications $(66) \Longrightarrow(67)$ and $(68) \Longrightarrow(69)$. The assertions of (66) and (68) follow from Lemma 5.4 given at the beginning of this section, but the work requires some effort to prepare. The implications are, on the other hand, easy, and so we proceed with those first. We shall then immediately return to the task of proving the assertions of (66) and (68).

5.2.1. Proof that (66) $\Longrightarrow$ (67). Observe

$$
\sum_{K^{-} \in Y} \operatorname{deg}_{\Lambda}\left(K^{-}\right)=\sum_{x \in X} \operatorname{deg}_{\Lambda}(x)
$$

Now, denote by $X_{\text {good }}$ the set of vertices $x \in X$ for which (66) holds. Then,

$$
\begin{aligned}
& \sum_{K^{-} \in Y} \operatorname{deg}_{\Lambda}\left(K^{-}\right)=\sum_{x \in X} \operatorname{deg}_{\Lambda}(x) \geq \sum_{x \in X_{\text {good }}} \operatorname{deg}_{\Lambda}(x) \stackrel{(66)}{\geq} \frac{\alpha^{\left(\begin{array}{c}
k-1 \\
2
\end{array}\right)}}{\ell^{k-1}}|Y|\left(1-2 \delta^{\frac{1}{36(k-1)}}\right)\left|X_{\text {good }}\right| \\
& \quad \stackrel{(66)}{\geq} \frac{\alpha^{\left(\begin{array}{c}
k-1 \\
2
\end{array}\right)}}{\ell^{k-1}}|Y|\left(1-2 \delta^{\frac{1}{36(k-1)}}\right)\left(1-\delta^{1 / 36}\right)|X| \stackrel{(55)}{\geq}\left(\frac{\alpha^{\left(\frac{k-1}{2}\right)}}{\ell^{k-1}}|X|\right)|Y|\left(1-\delta^{\frac{1}{37(k-1)}}\right),
\end{aligned}
$$

as promised in (67).

5.2.2. Proof that (68) $\Longrightarrow$ (69). Recall that we are supposed to bound $\sum_{K^{-} \in Y} \operatorname{deg}_{\Lambda}\left(K^{-}\right)^{2}$. To this end, we first observe that

$$
\sum_{\{x, y\} \in\left(\begin{array}{c}
X \\
2
\end{array}\right)} \operatorname{deg}_{\Lambda}(x, y)=\sum_{K^{-} \in Y}\left(\begin{array}{c}
\operatorname{deg}_{\Lambda}\left(K^{-}\right) \\
2
\end{array}\right) \stackrel{(55)}{=}\left(\frac{1}{2}-o(1)\right) \sum_{K^{-} \in Y} \operatorname{deg}_{\Lambda}\left(K^{-}\right)^{2},
$$

where $o(1) \rightarrow 0$ as $n \rightarrow \infty$. It therefore suffices to work with the sum $\sum_{\{x, y\} \in\left(\begin{array}{l}X \\ 2\end{array}\right)} \operatorname{deg}_{\Lambda}(x, y)$. 
Denote by $\left(\begin{array}{l}X \\ 2\end{array}\right)_{\text {good }}$ the set of pairs $\{x, y\} \in\left(\begin{array}{c}X \\ 2\end{array}\right)$ for which (68) holds. For pairs $\{x, y\} \in$ $\left(\begin{array}{l}X \\ 2\end{array}\right) \backslash\left(\begin{array}{l}X \\ 2\end{array}\right)_{\text {good }}$, we observe $\operatorname{deg}_{\Lambda}(x, y) \leq \operatorname{deg}_{\Pi}(x, y)$ (since $\Lambda \subseteq \Pi$ ) where we recall from Fact 5.2 that $\Pi$ is $\left(\ell^{1-k}, \varepsilon^{1 / 3}\right)$-regular. As such (and similarly to (61)), we have that all but $4 \varepsilon^{1 / 3} n^{2}$ pairs $\{x, y\} \in\left(\begin{array}{c}X \\ 2\end{array}\right)$ satisfy

$$
\operatorname{deg}_{\Pi}(x, y)=\left(\frac{1}{\ell^{k-1}} \pm \varepsilon^{1 / 3}\right)^{2}|Y| \stackrel{(55)}{<} \frac{2}{\ell^{2(k-1)}}|Y| .
$$

Denote by $\left(\begin{array}{c}X \\ 2\end{array}\right)_{\text {fair }}$ the set of pairs $\{x, y\} \in\left(\begin{array}{c}X \\ 2\end{array}\right)$ for which (71) holds.

Returning to (70), we then see the sum $\sum_{\{x, y\} \in\left(\begin{array}{l}X \\ 2\end{array}\right)} \operatorname{deg}_{\Lambda}(x, y)$ equals

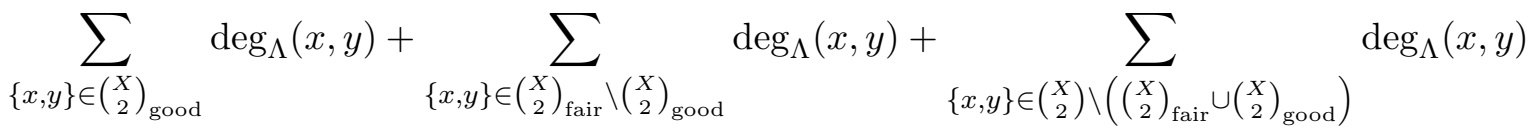

$$
\begin{aligned}
& \leq \sum_{\{x, y\} \in\left(\begin{array}{l}
X \\
2
\end{array}\right)_{\text {good }}} \operatorname{deg}_{\Lambda}(x, y)+\sum_{\{x, y\} \in\left(\begin{array}{l}
X \\
2
\end{array}\right)_{\text {fair }} \backslash\left(\begin{array}{l}
X \\
2
\end{array}\right)_{\text {good }}} \operatorname{deg}_{\Pi}(x, y)+|Y|\left|\left(\begin{array}{c}
X \\
2
\end{array}\right) \backslash\left(\begin{array}{c}
X \\
2
\end{array}\right)_{\text {fair }}\right| \\
& \stackrel{(68),(71)}{\leq}\left(\frac{\alpha^{\left(\begin{array}{c}
k-1 \\
2
\end{array}\right)}}{\ell^{k-1}}\right)^{2}|Y|\left(1+2 \delta^{\frac{1}{36(k-1)}}\right)\left|\left(\begin{array}{c}
X \\
2
\end{array}\right)_{\text {good }}\right|+\frac{2}{\ell^{2(k-1)}}|Y|\left|\left(\begin{array}{c}
X \\
2
\end{array}\right) \backslash\left(\begin{array}{c}
X \\
2
\end{array}\right)_{\text {good }}\right|+4 \varepsilon^{1 / 3} n^{2}|Y| \\
& \stackrel{(68)}{\leq}\left(\frac{\alpha^{\left(\begin{array}{c}
k-1 \\
2
\end{array}\right)}}{\ell^{k-1}}\right)^{2}|Y|\left(1+2 \delta^{\frac{1}{36(k-1)}}\right)\left(\begin{array}{l}
n \\
2
\end{array}\right)+2 \delta^{1 / 36} \frac{n^{2}}{\ell^{2(k-1)}}|Y|+4 \varepsilon^{1 / 3} n^{2}|Y| \\
& \leq\left(\frac{\alpha^{\left(\begin{array}{c}
k-1 \\
2
\end{array}\right)}}{\ell^{k-1}}\right)^{2}|Y| n^{2}\left(\frac{1}{2}+\delta^{\frac{1}{36(k-1)}}+2 \frac{\delta^{1 / 36}}{\alpha^{2\left(\begin{array}{c}
k-1 \\
2
\end{array}\right)}}+4 \varepsilon^{1 / 3} \frac{\ell^{2(k-1)}}{\alpha^{2\left(\begin{array}{c}
k-1 \\
2
\end{array}\right)}}\right) \stackrel{(55)}{\leq}\left(\frac{\alpha^{\left(\begin{array}{c}
k-1 \\
2
\end{array}\right)}}{\ell^{k-1}}|X|\right)^{2}|Y|\left(\frac{1}{2}+\delta^{\frac{1}{37(k-1)}}\right),
\end{aligned}
$$

so that, from (70), we have

$$
\sum_{K^{-} \in Y} \operatorname{deg}_{\Lambda}\left(K^{-}\right)^{2} \leq\left(\frac{\alpha^{\left(\begin{array}{c}
k-1 \\
2
\end{array}\right)}}{\ell^{k-1}}|X|\right)^{2}|Y|\left(1+2 \delta^{\frac{1}{37(k-1)}}\right)(1+o(1)) \stackrel{(55)}{<}\left(\frac{\alpha^{\left(\begin{array}{c}
k-1 \\
2
\end{array}\right)}}{\ell^{k-1}}|X|\right)^{2}|Y|\left(1+3 \delta^{\frac{1}{37(k-1)}}\right),
$$

as promised in (69).

5.3. Proofs of (66) and (68) - setting up the proofs. We claim that (66) and (68) are direct applications of the following auxiliary graph counting lemma.

Lemma 5.4 (A 'two graphs' counting lemma). For all integers $t$ and constants $\lambda_{0} \geq 0$ there exists $\delta_{0, \lambda_{0}}>0$ so that for all $0<\delta_{\lambda_{0}} \leq \delta_{0, \lambda_{0}}$ and $p>0$ there exists $\varepsilon_{p}>0$ so that the following holds:

Let

$$
\mathfrak{L}=\bigcup_{1 \leq i<j \leq t} \mathfrak{L}^{i j} \subseteq \mathfrak{P}=\bigcup_{1 \leq i<j \leq t} \mathfrak{P}^{i j}
$$

be $t$-partite graphs with common $t$-partition $U_{1} \cup \ldots \cup U_{t},\left|U_{i}\right|=m_{i}>m_{0}\left(t, \lambda_{0}, \delta_{\lambda}, p, \varepsilon_{p}\right)$, satisfying that for all $1 \leq i \neq j \leq t$,

(1) $\left|\mathfrak{L}^{i j}\right| /\left|\mathfrak{P}^{i j}\right| \stackrel{\text { def }}{=} \lambda_{i j} \geq \lambda_{0}$,

(2) all but $\delta_{\lambda_{0}} m_{i}^{2}$ pairs $u_{1}, u_{2} \in U_{i}$ satisfy

$$
\operatorname{deg}_{\mathfrak{L}^{i j}}\left(u_{1}, u_{2}\right)=\left(\lambda_{i j} p\right)^{2} m_{j}\left(1 \pm \delta_{\lambda_{0}}\right),
$$


(3) the graph $\mathfrak{P}^{i j}$ is $\left(p, \varepsilon_{p}\right)$-regular.

Then,

$$
\left|\mathcal{K}_{t}^{(2)}(\mathfrak{L})\right|=\left(\prod_{1 \leq i<j \leq t} \lambda_{i j}\right) p^{\left(\begin{array}{c}
t \\
2
\end{array}\right)}\left(\prod_{i=1}^{t} m_{i}\right)\left(1 \pm \delta_{\lambda_{0}}^{\frac{1}{5 t}}\right) .
$$

We prove Lemma 5.4 in Section 6.

We now connect (66) and (68) with Lemma 5.4. Indeed, for arbitrary vertices $x, y \in X=V_{1}$, observe from Construction 5.1 that

$$
\operatorname{deg}_{\Lambda}(x)=\left|\mathcal{K}_{k-1}^{(2)}\left(L_{x}\right)\right| \quad \text { and } \quad \operatorname{deg}_{\Lambda}(x, y)=\left|\mathcal{K}_{k-1}^{(2)}\left(L_{x y}\right)\right|
$$

where

$$
L_{x}=\{\{u, v\} \in P:\{x, u, v\} \in \mathcal{H}\} \quad \text { and } \quad L_{x y}=L_{x} \cap L_{y}
$$

are the links $L_{x}$ and colinks $L_{x y}$ of $x$ and $\{x, y\}$, resp. (cf. (36) and (37)). In view of (73), we may prove (66) and (68) by respectively applying Lemma 5.4 to graphs $L_{x}$ (i.e. $\mathfrak{L}=L_{x}$ ) and $L_{x y}$ (i.e. $\left.\mathfrak{L}=L_{x y}\right), x, y \in X=V_{1}$, whenever it is appropriate to do so.

To further develop our plans for proving (66) and (68) by applying Lemma 5.4, we continue with some notation (some of which is similar to that used in Section 4).

Notation 5.5. Let $T$ be a fixed one or two-element subset of $X$. We set

$$
U_{i, T}=N_{P^{1 i}}(T)=\bigcap_{x \in T} N_{P^{1 i}}(x) \quad \text { and } \quad m_{i, T}=\left|U_{i, T}\right|, \quad 1<i \leq k .
$$

Set

and

$$
\mathfrak{P}_{T}^{i j}=P_{T}^{i j}=P^{i j}\left[N_{P^{1 i}}(T), N_{P^{1 j}}(T)\right], 1<i<j \leq k,
$$

$$
\mathfrak{P}_{T}=\bigcup_{1<i<j \leq k} \mathfrak{P}_{T}^{i j}=\bigcup_{1<i<j \leq k} P_{T}^{i j}=P_{T}
$$

Note that $\mathfrak{P}_{T}$ is $(k-1)$-partite with $(k-1)$-partition $U_{2, T} \cup \cdots \cup U_{k, T}$. Set

$$
\mathfrak{L}_{T}=L_{T}=\bigcap_{x \in T} L_{x}
$$

where $L_{x}, x \in T$, is given in (74). For $1<i<j \leq k$, set $\mathfrak{L}_{T}^{i j}=\mathfrak{L}_{T} \cap P^{i j}$ so that

$$
\mathfrak{L}_{T}=\bigcup_{1<i<j \leq k} \mathfrak{L}_{T}^{i j}
$$

is $(k-1)$-partite with $(k-1)$-partition $U_{2, T} \cup \cdots \cup U_{k, T}$. Note that $\mathfrak{L}_{T} \subseteq \mathfrak{P}_{T}$.

We now make further preparations by considering some constants. For $T \in\left(\begin{array}{c}X \\ 1\end{array}\right) \cup\left(\begin{array}{l}X \\ 2\end{array}\right)=$ $\left(\begin{array}{c}V_{1} \\ 1\end{array}\right) \cup\left(\begin{array}{c}V_{1} \\ 2\end{array}\right)$, set

$$
\lambda_{T}^{i j}=\frac{\left|\mathfrak{L}_{T}^{i j}\right|}{\left|\mathfrak{P}_{T}^{i j}\right|}, 1<i<j \leq k, \quad \lambda_{0}=\frac{\alpha^{3}}{2}, \quad \delta_{\lambda_{0}}=4 \delta^{1 / 7}, \quad p=\frac{1}{\ell}, \quad \varepsilon_{p}=4 \ell^{2} \varepsilon .
$$

Recall from the hierarchy in (55) that

$$
\frac{1}{k}, \alpha \gg \delta \geq \min \left\{\ell^{-1}, \delta\right\} \gg \varepsilon .
$$


As such, we are easily afforded the hierarchy

$$
\frac{1}{k-1}, \lambda_{0}=\frac{\alpha^{3}}{2} \gg \delta_{\lambda_{0}}=4 \delta^{1 / 7} \geq \min \left\{p=\ell^{-1}, \delta_{\lambda_{0}}=4 \delta^{1 / 7}\right\} \gg \varepsilon_{p}=4 \ell^{2} \varepsilon .
$$

As such, the quantification of constants in Lemma 5.4 implies that

constants $k-1, \lambda_{0}, \delta_{\lambda_{0}}, p, \varepsilon_{p}$ (defined in (76)) are sufficient for an application of Lemma 5.4.

We conclude our preparations with the following fact.

Fact 5.6. For $t=1,2$, all but $(k-1) 2^{t} \varepsilon\left(\begin{array}{c}n \\ t\end{array}\right)$ sets $T \in\left(\begin{array}{c}X \\ t\end{array}\right)=\left(\begin{array}{c}V_{1} \\ t\end{array}\right)$ satisfy that $\mathfrak{P}_{T}^{i j}$ is $\left(p, \varepsilon_{p}\right)$-regular for all $1<i<j \leq k$.

Indeed, we see from (39) and (41) that all but $(k-1) 2^{t} \varepsilon\left(\begin{array}{l}n \\ t\end{array}\right)$ sets $T \in\left(\begin{array}{c}X \\ t\end{array}\right)=\left(\begin{array}{c}V_{1} \\ t\end{array}\right)$ satisfy that for all $1<i \leq k$,

$$
m_{i, T}=\left|U_{i, T}\right|=\left(\frac{1}{\ell} \pm \varepsilon\right)^{t} n=\frac{n}{\ell^{t}}(1 \pm \ell t \varepsilon) .
$$

As a consequence (cf. (39), (41)) these same sets $T \in\left(\begin{array}{c}X \\ t\end{array}\right)=\left(\begin{array}{c}V_{1} \\ t\end{array}\right), t=1,2$, satisfy that for all $1<i<j \leq k, \mathfrak{P}_{T}^{i j}$ is $\left(\ell^{-1},(2 \ell)^{t} \varepsilon\right)$-regular, or in the language above, $\mathfrak{P}_{T}^{i j}$ is $\left(p, \varepsilon_{p}\right)$-regular, $1<i<j \leq k$.

We now prove (66) and (68), in reverse order.

5.4. Proof of (68). We wish to apply Lemma 5.4 to the graphs $\mathfrak{L}=L_{x y},\{x, y\} \in\left(\begin{array}{c}X \\ 2\end{array}\right)=\left(\begin{array}{c}V_{1} \\ 2\end{array}\right)$. The following claims qualify the pairs for which this end is appropriate.

Claim 5.7. All but $3 \delta^{1 / 3}\left(\begin{array}{c}k-1 \\ 2\end{array}\right)\left(\begin{array}{l}n \\ 2\end{array}\right)$ pairs $\{x, y\} \in\left(\begin{array}{c}X \\ 2\end{array}\right)=\left(\begin{array}{c}V_{1} \\ 2\end{array}\right)$ satisfy

$$
\lambda_{x y}^{i j} \stackrel{(76)}{=} \frac{\left|\mathfrak{L}_{x y}^{i j}\right|}{\left|\mathfrak{P}_{x y}^{i j}\right|}=\alpha^{2}\left(1 \pm 4 \delta^{1 / 3}\right) \stackrel{(55)}{\geq} \frac{\alpha^{3}}{2} \stackrel{(76)}{=} \lambda_{0}
$$

for all $1<i<j \leq k$.

Claim 5.8. All but $2 \delta^{1 / 6}(k-1)^{2}\left(\begin{array}{c}n \\ 2\end{array}\right)$ pairs $\{x, y\} \in\left(\begin{array}{c}X \\ 2\end{array}\right)=\left(\begin{array}{c}V_{1} \\ 2\end{array}\right)$ satisfy the following property: all but $\delta_{\lambda_{0}}\left(\begin{array}{c}m_{i,\{x, y\}} \\ 2\end{array}\right)$ pairs $\{a, b\} \in\left(\begin{array}{c}U_{i,\{x, y\}} \\ 2\end{array}\right)$ satisfy

$$
\operatorname{deg}_{\mathfrak{L}_{x y}^{i j}}(a, b)=\left(\lambda_{x y}^{i j} p\right)^{2} m_{j,\{x, y\}}\left(1 \pm \delta_{\lambda_{0}}\right)
$$

for all $1<i \neq j \leq k$.

Before verifying Claims 5.7 and 5.8, let us use them to prove (68).

Claims 5.7 and 5.8 confirm that the hypothesis of Lemma 5.4 is met by the graph $\mathfrak{L}_{x y}$ for 'most' choices of $\{x, y\} \in\left(\begin{array}{c}X \\ 2\end{array}\right)=\left(\begin{array}{c}V_{1} \\ 2\end{array}\right)$. Indeed, Claim 5.7 confirms that Property (1) of Lemma 5.4 is met for all but $3 \delta^{1 / 3}\left(\begin{array}{c}k-1 \\ 2\end{array}\right)\left(\begin{array}{c}n \\ 2\end{array}\right)$ pairs $\{x, y\} \in\left(\begin{array}{c}V_{1} \\ 2\end{array}\right)$. Claim 5.8 confirms that Property (2) of Lemma 5.4 is met for all but an additional $2(k-1)^{2} \delta^{1 / 6}\left(\begin{array}{c}n \\ 2\end{array}\right)$ pairs $\{x, y\} \in\left(\begin{array}{c}V_{1} \\ 2\end{array}\right)$. By Fact 5.6, we see that all but $4(k-1) \varepsilon\left(\begin{array}{c}n \\ 2\end{array}\right)$ pairs $\{x, y\} \in\left(\begin{array}{c}V_{1} \\ 2\end{array}\right)$ satisfy Property (3) of Lemma 5.4. Finally, (78) confirms that our set of constants is sufficient for an application of Lemma 5.4. As such, for all but

$$
3 \delta^{1 / 3}\left(\begin{array}{c}
k-1 \\
2
\end{array}\right)\left(\begin{array}{l}
n \\
2
\end{array}\right)+2 \delta^{1 / 6}(k-1)^{2}\left(\begin{array}{l}
n \\
2
\end{array}\right)+4(k-1) \varepsilon\left(\begin{array}{l}
n \\
2
\end{array}\right) \leq 9 \delta^{1 / 6} k^{2} n^{2} \stackrel{(55)}{\leq} \delta^{1 / 8} n^{2}
$$


pairs $\{x, y\} \in\left(\begin{array}{c}X \\ 2\end{array}\right)=\left(\begin{array}{c}V_{1} \\ 2\end{array}\right)$, the graphs $\mathfrak{L}_{x y}=L_{x y}$ and $\mathfrak{P}_{x y}=P_{x y}$ satisfy the hypothesis of Lemma 5.4 with appropriate constants. Fixing one such pair $\{x, y\} \in\left(\begin{array}{c}X \\ 2\end{array}\right)=\left(\begin{array}{c}V_{1} \\ 2\end{array}\right)$, Lemma 5.4 yields

$$
\begin{aligned}
& \operatorname{deg}_{\Lambda}(x, y) \stackrel{(73)}{=}\left|\mathcal{K}_{k-1}^{(2)}\left(L_{x y}\right)\right|=\left|\mathcal{K}_{k-1}^{(2)}\left(\mathfrak{L}_{x y}\right)\right|=\left(\prod_{1<i<j \leq k} \lambda_{x y}^{i j}\right) p^{\left(\begin{array}{c}
k-1 \\
2
\end{array}\right)}\left(\prod_{1<i \leq k} m_{i, x y}\right)\left(1 \pm \delta_{\lambda_{0}}^{\frac{1}{5(k-1)}}\right) \\
& \text { (75), (76), (79), (80) }\left[\alpha^{2}\left(1 \pm 4 \delta^{1 / 3}\right)\right]^{\left(\begin{array}{c}
k-1 \\
2
\end{array}\right)} \frac{1}{\ell^{\left(\begin{array}{c}
k-1 \\
2
\end{array}\right)}}\left[\frac{n}{\ell^{2}}(1 \pm 2 \ell \varepsilon)\right]^{k-1}\left[1 \pm\left(4 \delta^{1 / 7}\right)^{\frac{1}{5(k-1)}}\right] \\
& =\left(\frac{\alpha^{2}}{\ell}\right)^{\left(\begin{array}{c}
k-1 \\
2
\end{array}\right)}\left(\frac{n}{\ell^{2}}\right)^{k-1}\left(1 \pm \delta^{\frac{1}{36(k-1)}}\right)
\end{aligned}
$$

as promised in (68).

It now remains to prove Claims 5.7 and 5.8. Claims 5.7 and 5.8 are ensured by Propositions 4.5 and 4.6, resp., from Section 4. We emphasize the following remark for future reference.

Remark 5.9. Claims 5.7 and 5.8 are guaranteed by applying Statement (2) of Proposition 4.5 and Statement (2)-(a) of Proposition 4.6, resp.

In the immediate sequel, we easily (and simultaneously) check that these lemmas may be applied in our current context. Afterwards, we confirm that Propositions 4.5 and 4.6 indeed yield Claims 5.7 and 5.8 .

5.4.1. Applying Propositions 4.5 and 4.6. Fix $1<i<j \leq k$. We check that Statement (2) of Proposition 4.5 and Statement (2)-(a) of Proposition 4.6 (see Section 4) may be applied to $\mathcal{H}^{1 i j}$ and $P^{1 i} \cup P^{1 j} \cup P^{i j}$. Note that our hypothesis in Theorem 2.7, the Counting Lemma, includes that $\mathcal{H}^{1 i j}$ and $P^{1 i} \cup P^{1 j} \cup P^{i j}$ satisfy the assumptions in Setup 2.1 with constants $\alpha, \ell$ and $\varepsilon$, as required by Statement (2) of Proposition 4.5 and Statement (2)-(a) of Proposition 4.6. Moreover, our hypothesis in Theorem 2.7 includes that $\mathcal{H}^{1 i j}$ is $(\alpha, \delta)$-minimal w.r.t. $P^{1 i} \cup P^{1 j} \cup$ $P^{i j}$, or in the language of Section $4, \mathcal{H}^{1 i j}$ is $(\alpha, \delta)_{2}$-minimal with respect to $P^{1 i} \cup P^{1 j} \cup P^{i j}$, as required by Statement (2) of Proposition 4.5 and Statement (2)-(a) of Proposition 4.6. Our hypothesis in Theorem 2.7 also includes that our constants $\alpha, \delta, \ell, \varepsilon$ and $n$ satisfy the hierarchy in (55), and as such, satisfy the quantifications of Propositions 4.5 and 4.6. We conclude that Statement (2) of Proposition 4.5 and Statement (2)-(a) of Proposition 4.6 may be applied to $\mathcal{H}^{1 i j}$ and $P^{1 i} \cup P^{1 j} \cup P^{i j}$.

5.4.2. Proof of Claim 5.7. We simply apply Statement (2) of Proposition 4.5 for fixed $1<i<$ $j \leq k$. This statement guarantees that all but $3 \delta^{1 / 3}\left(\begin{array}{c}n \\ 2\end{array}\right)$ pairs $\{x, y\} \in\left(\begin{array}{c}X \\ 2\end{array}\right)=\left(\begin{array}{c}V_{1} \\ 2\end{array}\right)$ satisfy

$$
\lambda_{x y}^{i j} \stackrel{(76)}{=} \frac{\left|\mathfrak{L}_{x y}^{i j}\right|}{\left|\mathfrak{P}_{x y}^{i j}\right|}=\frac{\left|L_{x y}^{i j}\right|}{\left|P_{x y}^{i j}\right|}=\alpha^{2}\left(1 \pm 4 \delta^{1 / 3}\right) .
$$

Thus, all but $3 \delta^{1 / 3}\left(\begin{array}{c}k-1 \\ 2\end{array}\right)\left(\begin{array}{l}n \\ 2\end{array}\right)$ pairs satisfy the above inequalities for all $1<i<j \leq k$. 
5.4.3. Proof of Claim 5.8. We shall use Statement (2)-(a) of Proposition 4.6 in the context of a proof by contradiction.

Assume, on the contrary, there exist $2 \delta^{1 / 6}(k-1)^{2}\left(\begin{array}{c}n \\ 2\end{array}\right)$ pairs $\{x, y\} \in\left(\begin{array}{c}X \\ 2\end{array}\right)=\left(\begin{array}{c}V_{1} \\ 2\end{array}\right)$ for which there exist $1<i \neq j \leq k$ for which some $\delta_{\lambda_{0}}\left(\begin{array}{c}m_{i,\{x, y\}} \\ 2\end{array}\right)$ pairs $\{a, b\} \in\left(\begin{array}{c}U_{i,\{x, y\}} \\ 2\end{array}\right)$ satisfying

$$
\operatorname{deg}_{\mathfrak{L}_{x y}^{i j}}(a, b) \neq\left(\lambda_{x y}^{i j} p\right)^{2} m_{j,\{x, y\}}\left(1 \pm \delta_{\lambda_{0}}\right) .
$$

As such, for some fixed pair of indices $1<i<j \leq k$, there must exist $2 \delta^{1 / 6}\left(\begin{array}{c}n \\ 2\end{array}\right)$ pairs $\{x, y\} \in\left(\begin{array}{l}X \\ 2\end{array}\right)$ (the set of which we denote by $X(i, j))$ for which there exist at least $\delta_{\lambda_{0}}\left(\begin{array}{c}m_{i,\{x, y\}} \\ 2\end{array}\right)$ pairs $\{a, b\} \in$ $\left(\begin{array}{c}U_{i,\{x, y\}} \\ 2\end{array}\right)$ (the set of which we denote by $U(i, x, y)$ ) satisfying

$$
\operatorname{deg}_{\mathfrak{L}_{x y}^{i j}}(a, b) \neq\left(\lambda_{x y}^{i j} p\right)^{2} m_{j,\{x, y\}}\left(1 \pm \delta_{\lambda_{0}}\right) .
$$

We show that the existence of the set $X(i, j)$, as described above, contradicts Statement (2)-(a) of Proposition 4.6.

Our first step is to refine the set $X(i, j)$ down to suitable pairs. Denote by $X^{\prime}(i, j) \subseteq X(i, j)$ the set of those pairs $\{x, y\}$ for which

$$
\lambda_{x y}^{i j}=\alpha^{2}\left(1 \pm 4 \delta^{1 / 3}\right) \quad \text { and } \quad m_{i,\{x, y\}}, m_{j,\{x, y\}}=\left(\frac{1}{\ell} \pm \varepsilon\right)^{2} n .
$$

We claim

$$
\left|X^{\prime}(i, j)\right| \geq \delta^{1 / 6}\left(\begin{array}{l}
n \\
2
\end{array}\right)
$$

Indeed, by Claim 5.7, we lose $3 \delta^{1 / 3}\left(\begin{array}{c}k-1 \\ 2\end{array}\right)\left(\begin{array}{c}n \\ 2\end{array}\right)$ pairs from $X(i, j)$ on account of the left condition of (85) failing. By the $\left(\ell^{-1}, \varepsilon\right)$-regularity of graphs $P^{1 i}$ and $P^{1 j}$, we lose another $8 \varepsilon\left(\begin{array}{l}n \\ 2\end{array}\right)$ pairs from $X(i, j)$ on account of the right condition of (85) failing. This shows

$$
\left|X^{\prime}(i, j)\right| \geq|X(i, j)|-3 \delta^{1 / 3}\left(\begin{array}{c}
k-1 \\
2
\end{array}\right)\left(\begin{array}{l}
n \\
2
\end{array}\right)-8 \varepsilon\left(\begin{array}{l}
n \\
2
\end{array}\right) \geq|X(i, j)|-4 \delta^{1 / 3}\left(\begin{array}{c}
k-1 \\
2
\end{array}\right)\left(\begin{array}{c}
n \\
2
\end{array}\right),
$$

where the last inequality holds with $\varepsilon \ll \delta$ (cf. (55)). On account of our assumption that $|X(i, j)| \geq$ $2 \delta^{1 / 6}\left(\begin{array}{l}n \\ 2\end{array}\right)$, we see that (86) holds with $\delta>0$ sufficiently small (cf. (55)).

We claim the set

$$
C_{4}(1, i)=\left\{(\{x, y\},\{a, b\}):\{x, y\} \in X^{\prime}(i, j),\{a, b\} \in U(i, x, y)\right\} \subseteq \mathcal{K}_{2,2}^{(2)}\left(P^{1 i}\right)
$$

is in contradiction with Statement (2)-(a) of Proposition 4.6. (Note that every element $(\{x, y\},\{a, b\}) \in$ $C_{4}(1, i)$ corresponds to a copy of $C_{4}$, with vertices $x, y, a, b$ and edges $\{x, a\},\{a, y\},\{y, b\},\{b, x\}$, where $x, y \in V_{1}$ and $a, b \in V_{i}$.) In particular, we claim that

$$
\left|C_{4}(1, i)\right|>3 \delta^{1 / 3}\left|\mathcal{K}_{2,2}^{(2)}\left(P^{1 i}\right)\right|
$$

and that for each $(\{x, y\},\{a, b\}) \in C_{4}(1, i)$,

$$
\operatorname{deg}_{L_{x y}^{i j}}(a, b) \neq\left(\frac{\alpha^{2}}{\ell}\right)^{2} \frac{n}{\ell^{2}}\left(1 \pm 3 \delta^{1 / 3}\right) .
$$

Then (87) and (88) are in violation with Statement (2)-(a) of Proposition 4.6. As such, establishing (87) and (88) shows that our assumption in (83) is incorrect, and therefore, proves Claim 5.8. 
To see (87), note that, account of (84)-(86), we have

$$
\left|C_{4}(1, i)\right| \geq \delta^{1 / 6}\left(\begin{array}{l}
n \\
2
\end{array}\right) \times \delta_{\lambda_{0}}\left(\begin{array}{c}
\left(\frac{1}{\ell}-\varepsilon\right)^{2} n \\
2
\end{array}\right) \stackrel{(55),(76)}{\geq} \delta^{13 / 42} \frac{n^{4}}{2 \ell^{4}} \stackrel{(43),(55)}{>} 3 \delta^{1 / 3}\left|\mathcal{K}_{2,2}^{(2)}\left(P^{1 i}\right)\right| .
$$

We now verify (88). Fix $(\{x, y\},\{a, b\}) \in C_{4}(1, i)$. Since

$$
\operatorname{deg}_{L_{x y}^{i j}}(a, b)=\operatorname{deg}_{\mathfrak{L}_{x y}^{i j}}(a, b) \neq\left(\lambda_{x y}^{i j} p\right)^{2} m_{j,\{x, y\}}\left(1 \pm \delta_{\lambda_{0}}\right),
$$

we have either

$$
\operatorname{deg}_{L_{x y}^{i j}}(a, b)<\left(\lambda_{x y}^{i j} p\right)^{2} m_{j,\{x, y\}}\left(1-\delta_{\lambda_{0}}\right) \quad \text { or } \quad \operatorname{deg}_{L_{x y}^{i j}}(a, b)>\left(\lambda_{x y}^{i j} p\right)^{2} m_{j,\{x, y\}}\left(1+\delta_{\lambda_{0}}\right) .
$$

Without loss of generality, we assume the former inequality holds and will prove that the former inequality implies

$$
\operatorname{deg}_{L_{x y}^{i j}}(a, b)<\left(\frac{\alpha^{2}}{\ell}\right)^{2} \frac{n}{\ell^{2}}\left(1-3 \delta^{1 / 3}\right) .
$$

This will complete our proof of (88).

Indeed, by (85), where $\lambda_{x y}^{i j}=\alpha^{2}\left(1 \pm 4 \delta^{1 / 3}\right)$, and since in (76), where we set $\delta_{\lambda_{0}}=4 \delta^{1 / 7}$ and $p=1 / \ell$, we have

$$
\begin{aligned}
& \operatorname{deg}_{L_{x y}^{i j}}(a, b)<\left(\lambda_{x y}^{i j} p\right)^{2} m_{j,\{x, y\}}\left(1-\delta_{\lambda_{0}}\right) \leq\left(\frac{\alpha^{2}}{\ell}\left(1+4 \delta^{1 / 3}\right)\right)^{2}\left(\frac{1}{\ell}+\varepsilon\right)^{2} n\left(1-4 \delta^{1 / 7}\right) \\
&<\left(\frac{\alpha^{2}}{\ell}\right)^{2} \frac{n}{\ell^{2}}\left(1-3 \delta^{1 / 3}\right),
\end{aligned}
$$

where we used $0<\varepsilon \ll \ell^{-1}, \delta$ and $\delta>0$ sufficiently small, as given in (55). This proves (90).

5.5. Proof of (66). The proof of (66) is nearly identical to what we did above in Section 5.4, save one detail: we argue that all steps above can be done for a 'typical' vertex $x \in X=V_{1}$ rather than for a 'typical' pair $\{x, y\} \in\left(\begin{array}{c}X \\ 2\end{array}\right)=\left(\begin{array}{c}V_{1} \\ 2\end{array}\right)$. More formally, we assert the following claims.

Claim 5.10. All but $3 \delta^{1 / 3}\left(\begin{array}{c}k-1 \\ 2\end{array}\right) n$ vertices $x \in X=V_{1}$ satisfy

$$
\lambda_{x}^{i j} \stackrel{(76)}{=} \frac{\left|\mathfrak{L}_{x}^{i j}\right|}{\left|\mathfrak{P}_{x}^{i j}\right|}=\alpha\left(1 \pm 4 \delta^{1 / 3}\right) \stackrel{(55)}{\geq} \frac{\alpha^{3}}{2} \stackrel{(76)}{=} \lambda_{0}
$$

for all $1<i<j \leq k$.

Claim 5.11. All but $2(k-1)^{2} \delta^{1 / 6} n$ many vertices $x \in X=V_{1}$ satisfy the following property: all but $\delta_{\lambda_{0}}\left(\begin{array}{c}m_{i, x} \\ 2\end{array}\right)$ pairs $\{a, b\} \in U_{i, x}$ satisfy

$$
\operatorname{deg}_{\mathfrak{L}_{x}^{i j}}(a, b)=\left(\lambda_{x}^{i j} p\right)^{2} m_{j, x}\left(1 \pm \delta_{\lambda_{0}}\right)
$$

$$
\text { for all } 1<i \neq j \leq k \text {. }
$$

Precisely as we did in Section 5.4, Claims 5.10 and 5.11, Fact 5.6 and the hierarchy in (77) say that for all but

$$
3 \delta^{1 / 3}\left(\begin{array}{c}
k-1 \\
2
\end{array}\right) n+2(k-1)^{2} \delta^{1 / 6} n+2(k-1) \varepsilon n \leq 7 \delta^{1 / 6} k^{2} n^{2} \stackrel{(55)}{\leq} \delta^{1 / 8} n
$$


vertices $x \in X=V_{1}$, the graphs $\mathfrak{L}_{x}=L_{x}$ and $\mathfrak{P}_{x}=P_{x}$ satisfy the hypothesis of Lemma 5.4 with appropriate constants. Fixing one such vertex $x \in X=V_{1}$, Lemma 5.4 yields (with $t=k-1)$

$$
\begin{gathered}
\operatorname{deg}_{\Lambda}(x) \stackrel{(73)}{=}\left|\mathcal{K}_{k-1}^{(2)}\left(L_{x}\right)\right|=\left|\mathcal{K}_{k-1}^{(2)}\left(\mathfrak{L}_{x}\right)\right|=\left(\prod_{1<i<j \leq k} \lambda_{x}^{i j}\right) p^{\left(\begin{array}{c}
k-1 \\
2
\end{array}\right)}\left(\prod_{1<i \leq k} m_{i, x}\right)\left(1 \pm \delta_{\lambda_{0}}^{\frac{1}{5(k-1)}}\right) \\
\stackrel{(75),(76),(79),(91)}{=}\left[\alpha\left(1 \pm 4 \delta^{1 / 3}\right)\right]^{\left(\begin{array}{c}
k-1 \\
2
\end{array}\right)} \frac{1}{\ell^{\left(\frac{k-1}{2}\right)}}\left[\frac{n}{\ell}(1 \pm \ell \varepsilon)\right]^{k-1}\left[1 \pm\left(4 \delta^{1 / 7}\right)^{\frac{1}{5(k-1)}}\right] \\
=\left(\frac{\alpha}{\ell}\right)^{\left(\begin{array}{c}
k-1 \\
2
\end{array}\right)}\left(\frac{n}{\ell}\right)^{k-1}\left(1 \pm \delta^{\frac{1}{36(k-1)}}\right),
\end{gathered}
$$

as promised in (66).

As before, Claims 5.10 and 5.11 follow from Propositions 4.5 and 4.6, resp., from Section 4. We stress, importantly, that

$$
\begin{aligned}
& \text { we now seek to apply Statement (1) of Proposition } 4.5 \text { and Statement (1)-(a) } \\
& \text { of Proposition } 4.6
\end{aligned}
$$

(recall Remark 5.9). As such, verifying that we may apply these statements of Propositions 4.5 and 4.6 requires the additional attention of one small detail which we now consider. Fix $1<i<j \leq k$. We wish to repeat the same verification we did in Section 5.4.1. For this, we need that $\mathcal{H}^{1 i j}$ is $(\alpha, \delta)_{1}$-minimal w.r.t. $P^{1 i} \cup P^{1 j} \cup P^{i j}$, a condition not initially assumed in the hypothesis of Theorem 2.7, but which follows by an application of Proposition 4.4.

\section{Proof of Lemma 5.4}

In this section, we prove Lemma 5.4. For simplicity, we prove Lemma 5.4 in the special case that $\lambda=\lambda_{0}=\lambda_{i j}, 1 \leq i<j \leq t$ and $m_{i}=m$ for all $1 \leq i \leq t$. Note that we also applied Lemma 5.4 in essentially the same special case (cf. (79) and Claims 5.7 and 5.10).

Our proof follows by induction on $t$ where the base case $t=2$ is trivial. We assume Lemma 5.4 holds up through $t-1 \geq 2$ and consider Lemma 5.4 for $t \geq 3$. We do not wish to begin our fairly simple argument with a tedious determination of constants. As such, with integer $t$ given above, let constants $\lambda, \delta_{0, \lambda}, \delta_{\lambda}, p, \varepsilon_{p}$ and $m$ be given satisfying the hierarchy

$$
\frac{1}{t}, \lambda \gg \delta_{0, \lambda}>\delta_{\lambda} \geq \min \left\{\delta_{\lambda}, p\right\} \gg \varepsilon_{p} \gg \frac{1}{m}
$$

which we note is consistent with the quantification of Lemma 5.4. With constants $t, \lambda=\lambda_{0}=$ $\lambda_{i j}, 1 \leq i<j \leq t, \delta_{\lambda}, p, \varepsilon_{p}$ and $m$, let graphs

$$
\mathfrak{L}=\bigcup_{1 \leq i<j \leq t} \mathfrak{L}^{i j} \subseteq \mathfrak{P}=\bigcup_{1 \leq i<j \leq t} \mathfrak{P}^{i j}
$$

on $t$-partition

$$
U_{1} \cup \ldots \cup U_{t}, \quad\left|U_{1}\right|=\ldots=\left|U_{1}\right|=m
$$

be given as in the hypothesis of Lemma 5.4. We show

$$
\left|\mathcal{K}_{t}^{(2)}(\mathfrak{L})\right|=(\lambda p)^{\left(\begin{array}{l}
t \\
2
\end{array}\right)} m^{t}\left(1 \pm \delta_{\lambda}^{\frac{1}{5 t}}\right),
$$

as promised by Lemma 5.4 .

To prove Lemma 5.4, we first define auxiliary bipartite graphs $\mathbb{L} \subseteq \mathbb{P}$. As we see momentarily, these graphs formulate Lemma 5.4 in slightly different language. 
Construction 6.1. With t-partition $U_{1} \cup \cdots \cup U_{t}$ of graphs $\mathfrak{L}=\bigcup_{1 \leq i<j \leq t} \mathfrak{L}^{i j}$ and $\mathfrak{P}=$ $\bigcup_{1 \leq i<j \leq t} \mathfrak{P}^{i j}$, define auxiliary bipartite graphs $\mathbb{L} \subseteq \mathbb{P}$ with bipartition $A \cup B$ as follows:

$$
A=U_{1} \quad \text { and } \quad B=\mathcal{K}_{t-1}^{(2)}\left(\mathfrak{P}\left[U_{2}, \ldots, U_{t}\right]\right)=\mathcal{K}_{t-1}^{(2)}\left(\bigcup_{1<i<j \leq t} \mathfrak{P}^{i j}\right)
$$

where elements of $B$, each denoted by $K^{-}$, correspond to vertex sets of $(t-1)$-cliques $K_{t-1}^{(2)}$ in the $(t-1)$-partite graph $\mathfrak{P}\left[U_{2}, \ldots, U_{t}\right]=\bigcup_{1<i<j \leq t} \mathfrak{P}^{i j}$. Note that

$$
|A|=m \quad \text { and } \quad|B| \stackrel{\text { Fact } 1.2}{=} p^{\left(\begin{array}{c}
t-1 \\
2
\end{array}\right)} m^{t-1}\left(1 \pm \varepsilon_{p}^{\frac{1}{t-1}}\right) \text {. }
$$

- For $a \in A$ and $K^{-} \in B$,

$$
\left\{a, K^{-}\right\} \in \mathbb{P} \Longleftrightarrow\{a\} \cup K^{-} \in \mathcal{K}_{t}^{(2)}(\mathfrak{P}) \Longleftrightarrow K^{-} \subset N_{\mathfrak{P}}(a) \Longleftrightarrow K^{-} \in \mathcal{K}_{t-1}^{(2)}\left(\mathfrak{P}\left[N_{\mathfrak{P}}(a)\right]\right)
$$

where $\mathfrak{P}\left[N_{\mathfrak{P}}(a)\right]$ is the subgraph of $\mathfrak{P}$ induced on the $\mathfrak{P}$-neighborhood $N_{\mathfrak{P}}(a)=N_{\mathfrak{P}^{12}}(a) \cup$ $\cdots \cup N_{\mathfrak{P}^{1 t}}(a)$ of $a$.

- For $a \in A$ and $K^{-} \in B$,

$$
\left\{a, K^{-}\right\} \in \mathbb{L} \Longleftrightarrow K^{-} \subset N_{\mathfrak{L}}(a) \Longleftrightarrow K^{-} \in \mathcal{K}_{t-1}^{(2)}\left(\mathfrak{P}\left[N_{\mathfrak{L}}(a)\right]\right)
$$

where $\mathfrak{P}\left[N_{\mathfrak{L}}(a)\right]$ is the subgraph of $\mathfrak{P}$ induced on the $\mathfrak{L}$-neighborhood $N_{\mathfrak{L}}(a)=N_{\mathfrak{L}^{12}}(a) \cup$ $\cdots \cup N_{\mathfrak{L}^{1 t}}(a)$ of $a$.

Note that $\mathfrak{L} \subseteq \mathfrak{P}$ implies $\mathbb{L} \subseteq \mathbb{P}$. The following fact is identical to Fact 5.2.

Fact 6.2. With $\varepsilon_{p}$ given in (94), the graph $\mathbb{P}$ is $\left(p^{t-1}, \varepsilon_{p}^{1 / 3}\right)$-regular.

We now make a few easy observations establishing connections between the graphs $\mathbb{L}$ and $\mathbb{P}$ and Lemma 5.4. For the purpose of stating these observations, set

$$
B_{\mathfrak{L}} \stackrel{\text { def }}{=} \mathcal{K}_{t-1}^{(2)}\left(\mathfrak{L}\left[U_{2}, \ldots, U_{t}\right]\right)=\mathcal{K}_{t-1}^{(2)}\left(\bigcup_{1<i<j \leq t} \mathfrak{L}^{i j}\right)
$$

and note that elements of $B_{\mathfrak{L}}$ correspond to $(t-1)$-cliques $K_{t-1}^{(2)}$ in the $(t-1)$-partite graph $\mathfrak{L}\left[U_{2}, \ldots, U_{t}\right]=\bigcup_{1<i<j \leq t} \mathfrak{L}^{i j}$.

\section{Observations.}

- Since $\mathfrak{L} \subseteq \mathfrak{P}, B_{\mathfrak{L}} \subseteq B$.

- By our Induction Hypothesis on Lemma 5.4 (for $t-1$ ),

$$
\begin{gathered}
\left|B_{\mathfrak{L}}\right|=\left|\mathcal{K}_{t-1}^{(2)}\left(\mathfrak{L}\left[U_{2}, \ldots, U_{t}\right]\right)\right|=\left|\mathcal{K}_{t-1}^{(2)}\left(\bigcup_{1<i<j \leq t} \mathfrak{L}^{i j}\right)\right| \\
=(\lambda p)^{\left(\begin{array}{c}
(t-1 \\
2
\end{array}\right)} m^{t-1}\left(1 \pm \delta_{\lambda}^{\frac{1}{5(t-1)}}\right) \stackrel{(94),(96)}{=} \lambda^{\left(\begin{array}{c}
(-1 \\
2
\end{array}\right)}|B|\left(1 \pm 2 \delta_{\lambda}^{\frac{1}{5(t-1)}}\right) . \\
\left|\mathcal{K}_{t}^{(2)}(\mathfrak{L})\right|=\sum_{K^{-} \in B_{\mathfrak{L}}} \operatorname{deg}_{\mathbb{L}}\left(K^{-}\right) .
\end{gathered}
$$


We see from (97) and (98) that, to prove Lemma 5.4, it suffices to analyze the terms in the sum (98). Proposition 6.3 does precisely this (and is similar to Proposition 5.3 of the preceding section).

Proposition 6.3. All but $2 \delta_{\lambda}^{1 / 12}|B|=2 \delta_{\lambda}^{1 / 12}\left|\mathcal{K}_{t-1}^{(2)}\left(\bigcup_{1<i<j \leq t} \mathfrak{P}^{i j}\right)\right|$ many vertices $K^{-} \in B=$ $\mathcal{K}_{t-1}^{(2)}\left(\bigcup_{1<i<j \leq t} \mathfrak{P}^{i j}\right)$ satisfy

$$
\operatorname{deg}_{\mathbb{L}}\left(K^{-}\right)=(\lambda p)^{t-1}|A|\left(1 \pm 2 \delta_{\lambda}^{1 / 12}\right)=(\lambda p)^{t-1} m\left(1 \pm 2 \delta_{\lambda}^{1 / 12}\right) .
$$

We defer the proof of Proposition 6.3 to Section 6.2 and proceed now with the easy confirmation that Lemma 5.4 follows from Proposition 6.3.

6.1. Proposition 6.3 $\Longrightarrow$ Lemma 5.4. Our proof that follows is quite similar to we saw when using Proposition 5.3 to prove Theorem 2.7.

We employ $\operatorname{deg}_{\mathbb{L}}\left(K^{-}\right) \leq \operatorname{deg}_{\mathbb{P}}\left(K^{-}\right)$for all $K^{-} \in B_{\mathfrak{L}}$ not satisfying (99). As such, we note from Fact 6.2 that all but $2 \varepsilon_{p}^{1 / 3}|B|$ vertices $K^{-} \in B$ satisfy

$$
\operatorname{deg}_{\mathbb{P}}\left(K^{-}\right)=p^{t-1}|A|\left(1 \pm \varepsilon_{p}^{1 / 3}\right) \stackrel{(94)}{\leq} 2 p^{t-1} m .
$$

Now, set

$$
B_{\mathbb{L}}=\left\{K^{-} \in B: K^{-} \text {satisfies (99) }\right\} \quad \text { and } \quad B_{\mathbb{P}}=\left\{K^{-} \in B: K^{-} \text {satisfies (100) }\right\} .
$$

Proposition 6.3 and (100) then imply

$$
\left|B_{\mathfrak{L}} \backslash B_{\mathbb{L}}\right| \leq\left|B \backslash B_{\mathbb{L}}\right| \leq 2 \delta_{\lambda}^{1 / 12}|B| \stackrel{(96)}{\leq} 4 \delta_{\lambda}^{1 / 12} p^{\left(\begin{array}{c}
t-1 \\
2
\end{array}\right)} m^{t-1}, \quad\left|B_{\mathfrak{L}} \backslash B_{\mathbb{P}}\right| \leq 2 \varepsilon_{p}^{1 / 3}|B| \leq 4 \varepsilon_{p}^{1 / 3} p^{\left(\begin{array}{c}
t-1 \\
2
\end{array}\right)} m^{t-1} .
$$

Now the proof of Lemma 5.4 is immediate. Returning to (98), we see

$$
(102)=\sum_{K^{-} \in\left(B_{\mathfrak{L}} \cap B_{\mathbb{L}}\right)} \operatorname{deg}_{\mathbb{L}}\left(K^{-}\right)+\sum_{K^{-} \in\left(B_{\mathbb{P}} \backslash B_{\mathbb{L}}\right) \cap B_{\mathfrak{L}}} \operatorname{deg}_{\mathbb{L}}\left(K^{-}\right)+\sum_{K^{-} \in\left(B_{\mathfrak{L}} \backslash\left(B_{\mathbb{L}} \cup B_{\mathbb{P}}\right)\right)} \operatorname{deg}_{\mathbb{L}}\left(K^{-}\right) .
$$

To obtain the formula for $\left|\mathcal{K}_{t}^{(2)}(\mathfrak{L})\right|$ promised in (95), we need to bound (102) from above and below. For the lower bound, we employ (99) in (102) to obtain

$$
\left|\mathcal{K}_{t}^{(2)}(\mathfrak{L})\right| \geq(\lambda p)^{t-1} m\left(1-2 \delta_{\lambda}^{1 / 12}\right)\left|B_{\mathfrak{L}} \cap B_{\mathbb{L}}\right|=(\lambda p)^{t-1} m\left(1-2 \delta_{\lambda}^{1 / 12}\right)\left(\left|B_{\mathfrak{L}}\right|-\left|B_{\mathfrak{L}} \backslash B_{\mathbb{L}}\right|\right) .
$$

By (97) and (101), we then see

$$
\left|\mathcal{K}_{t}^{(2)}(\mathfrak{L})\right| \geq(\lambda p)^{\left(\begin{array}{l}
t \\
2
\end{array}\right)} m^{t}\left(1-2 \delta_{\lambda}^{1 / 12}\right)\left(1-\delta_{\lambda}^{\frac{1}{5(t-1)}}-4 \frac{\delta_{\lambda}^{1 / 12}}{\lambda^{\left(\begin{array}{c}
t-1 \\
2
\end{array}\right)}}\right) \geq(\lambda p)^{\left(\begin{array}{l}
t \\
2
\end{array}\right)} m^{t}\left(1-\delta_{\lambda}^{\frac{1}{5 t}}\right),
$$

which holds with $t \geq 3$ and $0<\delta_{\lambda} \ll \lambda, t^{-1}$ sufficiently small in (94).

For the upper bound, we employ (99) and (100) in (102) to see

$$
\begin{gathered}
\left|\mathcal{K}_{t}^{(2)}(\mathfrak{L})\right| \leq(\lambda p)^{t-1} m\left(1+2 \delta_{\lambda}^{1 / 12}\right)\left|B_{\mathfrak{L}} \cap B_{\mathbb{L}}\right|+2 p^{t-1} m\left|\left(B_{\mathbb{P}} \backslash B_{\mathbb{L}}\right) \cap B_{\mathfrak{L}}\right|+m\left|B_{\mathfrak{L}} \backslash\left(B_{\mathbb{L}} \cup B_{\mathbb{P}}\right)\right| \\
\leq(\lambda p)^{t-1} m\left(1+2 \delta_{\lambda}^{1 / 12}\right)\left|B_{\mathfrak{L}}\right|+2 p^{t-1} m\left|B_{\mathfrak{L}} \backslash B_{\mathbb{L}}\right|+m\left|B_{\mathfrak{L}} \backslash B_{\mathbb{P}}\right| .
\end{gathered}
$$


Using (97) and (101), we obtain

$$
\begin{gathered}
\left|\mathcal{K}_{t}^{(2)}(\mathfrak{L})\right| \leq(\lambda p)^{\left(\begin{array}{l}
t \\
2
\end{array}\right)} m^{t}\left(1+2 \delta_{\lambda}^{1 / 12}\right)\left(1+\delta_{\lambda}^{\frac{1}{5(t-1)}}\right)+8 \delta_{\lambda}^{1 / 12} p^{\left(\begin{array}{c}
t \\
2
\end{array}\right)} m^{t}+4 \varepsilon_{p}^{1 / 3} p^{\left(\begin{array}{c}
t-1 \\
2
\end{array}\right)} m^{t} \\
=(\lambda p)^{\left(\begin{array}{l}
t \\
2
\end{array}\right)} m^{t}\left[\left(1+2 \delta_{\lambda}^{1 / 12}\right)\left(1+\delta_{\lambda}^{\frac{1}{5(t-1)}}\right)+\frac{8 \delta_{\lambda}^{1 / 12}}{\lambda^{\left(\begin{array}{c}
t \\
2
\end{array}\right)}}+\frac{4 \varepsilon_{p}^{1 / 3}}{p^{t-1}}\right] \stackrel{(94)}{\leq}(\lambda p)^{\left(\begin{array}{c}
t \\
2
\end{array}\right)} m^{t}\left(1+\delta_{\lambda}^{\frac{1}{5 t}}\right)
\end{gathered}
$$

which holds with $t \geq 3$ and the hierarchy in (94). This completes the proof of the induction step for Lemma 5.4 .

6.2. Proof of Proposition 6.3. All that remains is to prove Proposition 6.3. We do so by proving the following slightly stronger version of Proposition 6.3 (which is similar to how we handled Proposition 5.3 through Proposition 5.3*).

\section{Proposition 6.3*.}

- All but $4 \delta_{\lambda}^{1 / 3} m$ vertices $u \in A=U_{1}$ satisfy

$$
\operatorname{deg}_{\mathbb{L}}(u)=p^{\left(\begin{array}{c}
t-1 \\
2
\end{array}\right)}(\lambda p m)^{t-1}\left(1 \pm(4 t)^{2} \delta_{\lambda}^{1 / 3}\right) \stackrel{(94),(96)}{=}(\lambda p)^{t-1}|B|\left(1 \pm 2(4 t)^{2} \delta_{\lambda}^{1 / 3}\right) .
$$

In particular,

$$
\sum_{K^{-} \in B} \operatorname{deg}_{\mathbb{L}}\left(K^{-}\right)>\left((\lambda p)^{t-1}|A|\right)|B|\left(1-\delta_{\lambda}^{1 / 4}\right) .
$$

- All but $\delta_{\lambda} m^{2}$ pairs $u, v \in A=U_{1}$ satisfy

$$
\operatorname{deg}_{\mathbb{L}}(u, v)=p^{\left(\begin{array}{c}
2-1 \\
2
\end{array}\right)}\left((\lambda p)^{2} m\right)^{t-1}\left(1 \pm t^{2} \delta_{\lambda}\right) \stackrel{(94),(96)}{=}(\lambda p)^{2(t-1)}|B|\left(1 \pm 2 t^{2} \delta_{\lambda}\right) .
$$

In particular,

$$
\sum_{K^{-} \in B} \operatorname{deg}_{\mathbb{L}}\left(K^{-}\right)^{2} \leq\left((\lambda p)^{t-1}|A|\right)^{2}|B|\left(1+3 \delta_{\lambda}^{1 / 2}\right) \stackrel{(94)}{<}\left((\lambda p)^{t-1}|A|\right)^{2}|B|\left(1+\delta_{\lambda}^{1 / 4}\right) .
$$

It is easy to prove Proposition 6.3 from Proposition $6.3^{*}$. Indeed, using the approximate Cauchy-Schwarz inequality (that is, Fact 4.7), Proposition 6.3 is an immediately corollary of (104) and (106). (To see this, in Fact 4.7 set $a=(\lambda p)^{t-1}|A|, r=|B|$ and $\gamma=\delta_{\lambda}^{1 / 4}$ and let terms $a_{i}, 1 \leq i \leq r$, correspond to terms $\left.\operatorname{deg}_{\mathbb{L}}\left(K^{-}\right), K^{-} \in B\right)$. It therefore remains to prove Proposition $6.3^{*}$.

To prove Proposition $6.3^{*}$, we must show 4 things: the assertions of (103) and (105) and the implications $(103) \Longrightarrow(104)$ and $(105) \Longrightarrow(106)$. We proceed to first prove the implications and then shall prove the assertions.

6.2.1. Proof that (103) $\Longrightarrow$ (104). Observe that

$$
\sum_{K^{-} \in B} \operatorname{deg}_{\mathbb{L}}\left(K^{-}\right)=\sum_{u \in A} \operatorname{deg}_{\mathbb{L}}(u)
$$

Now, denote by $A_{\text {good }}$ the set of vertices $u \in A=U_{1}$ for which (103) holds. We then have

$$
\sum_{K^{-} \in B} \operatorname{deg}_{\mathbb{L}}\left(K^{-}\right)=\sum_{u \in A} \operatorname{deg}_{\mathbb{L}}(u) \geq \sum_{u \in A_{\text {good }}} \operatorname{deg}_{\mathbb{L}}(u) \stackrel{(103)}{\geq}(\lambda p)^{t-1}|B|\left(1-2(4 t)^{2} \delta_{\lambda}^{1 / 3}\right)\left|A_{\text {good }}\right|
$$




$$
\stackrel{\text { Prop. 6.3* }}{\geq}(\lambda p)^{t-1}|B|\left(1-2(4 t)^{2} \delta_{\lambda}^{1 / 3}\right)\left(1-4 \delta_{\lambda}^{1 / 3}\right)|A| \stackrel{(94)}{\geq}\left((\lambda p)^{t-1}|A|\right)|B|\left(1-\delta_{\lambda}^{1 / 4}\right),
$$

as promised in (104).

6.2.2. Proof that $(105) \Longrightarrow(106)$. Recall that we are supposed to bound $\sum_{K^{-} \in B} \operatorname{deg}_{\mathbb{L}}\left(K^{-}\right)^{2}$ from above. First we observe that

$$
\sum_{\{u, v\} \in\left(\begin{array}{c}
A \\
2
\end{array}\right)} \operatorname{deg}_{\mathbb{L}}(u, v)=\sum_{K^{-} \in B}\left(\begin{array}{c}
\operatorname{deg}_{\mathbb{L}}\left(K^{-}\right) \\
2
\end{array}\right) \stackrel{(94)}{=}\left(\frac{1}{2}-o(1)\right) \sum_{K^{-} \in B} \operatorname{deg}_{\mathbb{L}}\left(K^{-}\right)^{2},
$$

where $o(1) \rightarrow 0$ as $m \rightarrow \infty$. It therefore suffices to consider the sum $\sum_{\{u, v\} \in\left(\begin{array}{c}A \\ 2\end{array}\right)} \operatorname{deg}_{\mathbb{L}}(u, v)$.

Denote by $\left(\begin{array}{c}A \\ 2\end{array}\right)_{\text {good }}$ the set of all pairs $\{u, v\} \in\left(\begin{array}{c}A \\ 2\end{array}\right)$ for which (105) holds. For pairs $\{u, v\} \notin$ $\left(\begin{array}{l}A \\ 2\end{array}\right)_{\text {good }}$, we observe $\operatorname{deg}_{\mathbb{L}}(u, v) \leq \operatorname{deg}_{\mathbb{P}}(u, v)($ since $\mathbb{L} \subseteq \mathbb{P})$ where we recall from Fact 6.2 that $\mathbb{P}$ is $\left(p^{t-1}, \varepsilon_{p}^{1 / 3}\right)$-regular. As such (and similarly to (100)), we have that all but $4 \varepsilon_{p}^{1 / 3} m^{2}$ pairs $\{u, v\} \in\left(\begin{array}{c}A \\ 2\end{array}\right)$ satisfy

$$
\operatorname{deg}_{\mathbb{P}}(u, v)=\left(p^{t-1}+\varepsilon_{p}^{1 / 3}\right)^{2}|B| \stackrel{(94)}{\leq} 2 p^{2(t-1)}|B| .
$$

Denote by $\left(\begin{array}{c}A \\ 2\end{array}\right)_{\text {fair }}$ the set of all pairs $\{u, v\} \in\left(\begin{array}{c}A \\ 2\end{array}\right)$ for which (108) holds.

Returning to (107), we see the sum $\sum_{\{u, v\} \in\left(\begin{array}{l}A \\ 2\end{array}\right)} \operatorname{deg}_{\mathbb{L}}(u, v)$ equals

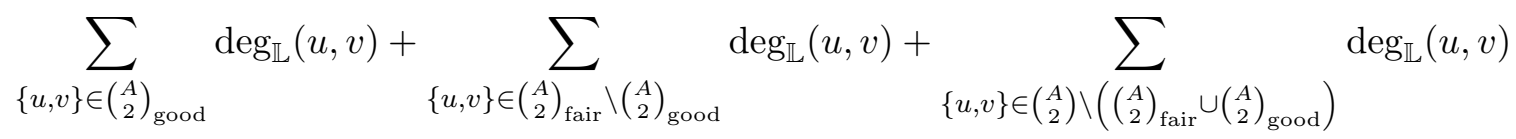

$$
\begin{aligned}
& \leq \sum_{\{u, v\} \in\left(\begin{array}{c}
A \\
2
\end{array}\right)_{\text {good }}} \operatorname{deg}_{\mathbb{L}}(u, v)+\sum_{\{u, v\} \in\left(\begin{array}{c}
A \\
2
\end{array}\right)_{\text {fair }} \backslash\left(\begin{array}{l}
A \\
2
\end{array}\right)_{\text {good }}} \operatorname{deg}_{\mathbb{P}}(u, v)+|B|\left|\left(\begin{array}{c}
A \\
2
\end{array}\right) \backslash\left(\begin{array}{c}
A \\
2
\end{array}\right)_{\text {fair }}\right|
\end{aligned}
$$

$$
\begin{gathered}
\underset{\text { Prop. 6.3*, (108) }}{\leq}(\lambda p)^{2(t-1)}|B|\left(1+2 t^{2} \delta_{\lambda}\right)\left|\left(\begin{array}{c}
A \\
2
\end{array}\right)_{\text {good }}\right|+2 p^{2(t-1)}|B|\left|\left(\begin{array}{c}
A \\
2
\end{array}\right) \backslash\left(\begin{array}{c}
A \\
2
\end{array}\right)_{\text {good }}\right|+4 \varepsilon_{p}^{1 / 3} m^{2}|B| \\
\stackrel{\text { Prop. 6.3* }}{\leq}(\lambda p)^{2(t-1)}|B|\left(1+2 t^{2} \delta_{\lambda}\right)\left(\begin{array}{c}
m \\
2
\end{array}\right)+2 \delta_{\lambda} p^{2(t-1)}|B| m^{2}+4 \varepsilon_{p}^{1 / 3} m^{2}|B| \\
\leq(\lambda p)^{2(t-1)}|B| m^{2}\left(\left(\frac{1}{2}+t^{2} \delta_{\lambda}\right)+2 \frac{\delta_{\lambda}}{\lambda^{2(t-1)}}+4 \frac{\varepsilon_{p}^{1 / 3}}{(\lambda p)^{2(t-1)}}\right) \stackrel{(94)}{<}\left((\lambda p)^{t-1}|A|\right)^{2}|B|\left(\frac{1}{2}+\delta_{\lambda}^{1 / 2}\right),
\end{gathered}
$$

so that, with (107), we have

$$
\sum_{K^{-} \in B} \operatorname{deg}_{\mathbb{L}}\left(K^{-}\right)^{2} \leq\left((\lambda p)^{t-1}|A|\right)^{2}|B|\left(1+2 \delta_{\lambda}^{1 / 2}\right)(1+o(1)) \stackrel{(94)}{<}\left((\lambda p)^{t-1}|A|\right)^{2}|B|\left(1+3 \delta_{\lambda}^{1 / 2}\right),
$$

as promised in (106).

It remains to prove (103) and (105). We begin with the latter. 
6.2.3. Proof of (105). For an arbitrary pair of vertices $u, v \in A=U_{1}$, observe

$$
\operatorname{deg}_{\mathbb{L}}(u, v)=\left|\mathcal{K}_{t-1}^{(2)}\left(\mathfrak{P}\left[N_{\mathfrak{L}}(u, v)\right]\right)\right|=\left|\mathcal{K}_{t-1}^{(2)}\left(\bigcup_{1<i<j \leq t} \mathfrak{P}^{i j}\left[N_{\mathcal{L}^{1 i}}(u, v), N_{\mathcal{L}^{1 j}}(u, v)\right]\right)\right|
$$

follows by our construction of graph $\mathbb{L}$. Estimating $\left|\mathcal{K}_{t-1}^{(2)}\left(\mathfrak{P}\left[N_{\mathfrak{L}}(u, v)\right]\right)\right|$ is, however, a mere application of Fact 1.2.

Indeed, the hypothesis of Lemma 5.4 gives that all but $\delta_{\lambda} m^{2}$ pairs $u, v \in U_{1}=A$ satisfy $\operatorname{deg}_{\mathcal{L}^{1 i}}(u, v)=(\lambda p)^{2} m\left(1 \pm \delta_{\lambda}\right), 1<i \leq t$. Fix one such pair $u, v \in U_{1}=A$. We claim $u, v$ satisfy the conclusion of Proposition 6.3*. Indeed, fix $1<i<j \leq t$ and observe

$$
\min \left\{\operatorname{deg}_{\mathfrak{L}^{1 i}}(u, v), \operatorname{deg}_{\mathfrak{L}^{1 j}}(u, v)\right\} \geq(\lambda p)^{2} m\left(1-\delta_{\lambda}\right) \stackrel{(94)}{\geq} \frac{1}{2}(\lambda p)^{2} m \stackrel{(94)}{\gg} \varepsilon_{p}^{1 / 2} m .
$$

Using (110), the $\left(p, \varepsilon_{p}\right)$-regularity of $\mathfrak{P}^{i j}$ implies that the graph $\mathfrak{P}^{i j}\left[N_{\mathfrak{L}^{1 i}}(u, v), N_{\mathfrak{L}^{1 j}}(u, v)\right]$ is $\left(p, \varepsilon_{p}^{1 / 2}\right.$ )-regular (see Fact 1.5, the Slicing Lemma, from [29]). As such, we apply Fact 1.2 to conclude

$\left|\mathcal{K}_{t-1}^{(2)}\left(\mathfrak{P}\left[N_{\mathfrak{L}}(u, v)\right]\right)\right|=p^{\left(\begin{array}{c}t-1 \\ 2\end{array}\right)}\left[(\lambda p)^{2} m\left(1 \pm \delta_{\lambda}\right)\right]^{t-1}\left(1 \pm \varepsilon_{p}^{\frac{1}{2(t-1)}}\right) \stackrel{(94)}{=} p^{\left(\begin{array}{c}t-1 \\ 2\end{array}\right)}\left[(\lambda p)^{2} m\right]^{t-1}\left(1 \pm t^{2} \delta_{\lambda}\right)$

which renders the result.

6.2.4. Proof of (103). We will follow essentially the same procedure as described above, but for single vertices $u \in A=U_{1}$ rather than pairs $u, v \in A=U_{1}$. Note however, in this case, Lemma 5.4 admits no hypothesis on $\operatorname{deg}_{\mathfrak{L}^{1 i}}(u)$ for single vertices $u \in A=U_{1}$. For this reason, we require the following fact.

Fact 6.4. With $\mathfrak{L}$ and $\mathfrak{P}$ given in Lemma 5.4, all but $4 \delta_{\lambda}^{1 / 3} m$ vertices $u \in U_{1}$ satisfy that for each $1<i \leq t$,

$$
\operatorname{deg}_{\mathfrak{L}^{1 i}}(u)=\lambda p m\left(1 \pm 4 \delta_{\lambda}^{1 / 3}\right) .
$$

Note that Fact 6.4 gives the analogue of Condition (2) of Lemma 5.4 for single vertices $u \in U_{1}$, $1 \leq i<t$. The proof of Fact 6.4 follows from Conditions (1) and (2) of Lemma 5.4 by a standard Cauchy-Schwarz argument. We omit the standard details. We now use Fact 6.4 to finish the proof of Proposition 6.3*.

Similarly to (109), for a fixed vertex $u \in A=U_{1}$, we have

$$
\operatorname{deg}_{\mathbb{L}}(u)=\left|\mathcal{K}_{t-1}^{(2)}\left(\mathfrak{P}\left[N_{\mathfrak{L}}(u)\right]\right)\right|=\left|\mathcal{K}_{t-1}^{(2)}\left(\bigcup_{1<i<j \leq t} \mathfrak{P}^{i j}\left[N_{\mathfrak{L}^{1 i}}(u), N_{\mathcal{L}^{1 j}}(u)\right]\right)\right| .
$$

Fact 6.4 ensures all but $4 \delta_{\lambda}^{1 / 3} m$ vertices $u \in U_{1}$ satisfy that for all $1<i<j \leq t$,

$$
\min \left\{\operatorname{deg}_{\mathfrak{L}^{1 i}}(u), \operatorname{deg}_{\mathfrak{L}^{1 j}}(u)\right\} \geq \lambda p m\left(1-4 \delta_{\lambda}^{1 / 3}\right) \stackrel{(94)}{\geq} \frac{1}{2} \lambda p m \stackrel{(94)}{\gg} \varepsilon_{p}^{1 / 2} m .
$$

Fix one such vertex $u \in U_{1}$. Similarly to (110), the $\left(p, \varepsilon_{p}\right)$-regularity of $\mathfrak{P}^{i j}, 1<i<j \leq t$, implies that the graph $\mathfrak{P}^{i j}\left[N_{\mathfrak{L}^{1 i}}(u) N_{\mathfrak{L}^{1 j}}(u)\right]$ is $\left(p, \varepsilon_{p}^{1 / 2}\right)$-regular. As such, we apply Fact 1.2 to conclude

$\left|\mathcal{K}_{t-1}^{(2)}\left(\mathfrak{P}\left[N_{\mathfrak{L}}(u)\right]\right)\right|=p^{\left(\frac{t-1}{2}\right)}\left[\lambda p m\left(1 \pm 4 \delta_{\lambda}^{1 / 3}\right)\right]^{t-1}\left(1 \pm \varepsilon_{p}^{\frac{1}{2(t-1)}}\right) \stackrel{(94)}{=} p^{\left(\frac{t-1}{2}\right)}(\lambda p m)^{t-1}\left(1 \pm(4 t)^{2} \delta_{\lambda}^{1 / 3}\right)$

which renders the result. 


\section{Proof of Statement (2) of Proposition 3.2}

In Section 2.4, we asserted that Statement (2) of Proposition 3.2 follows from Lemma 5.8 (cf. Algorithm A) of [11]. (Proposition 3.2 and Lemma 5.8 of [11] are very similar statements, with almost the same quantification.) In this section, we show that the earlier Lemma 5.8 implies Proposition 3.2. We begin by presenting Lemma 5.8 from [11], as well as upcoming Fact 7.3 that we shall also employ in our proof.

7.1. Background material. To state Lemma 5.8 of [11], we need the following concept.

Definition 7.1 $((\gamma, \delta, R)$-regular). Let bipartite graph $F$ have bipartition $U \cup W$. For given constants $\gamma, \delta>0$ and for given integer $R$, we say that $F$ is $(\gamma, \delta, R)$-regular if for all $U_{1}, \ldots, U_{R} \subseteq U$ and all $W_{1}, \ldots, W_{R} \subseteq W$ for which

$$
\left|\bigcup_{i=1}^{R}\left(U_{i} \times W_{i}\right)\right|>\delta|U||W|
$$

we have

$$
\left|F \cap \bigcup_{i=1}^{R}\left(U_{i} \times W_{i}\right)\right|=\gamma(1 \pm \delta)\left|\bigcup_{i=1}^{R} U_{i} \times W_{i}\right| .
$$

(Here, for a fixed $1 \leq i \leq R$, we define $U_{i} \times W_{i}$ to be $\left\{\left\{u_{i}, w_{i}\right\}: u_{i} \in U_{i}, w_{i} \in W_{i}\right\}$.)

Lemma 5.8 of [11] is then stated ${ }^{2}$ as follows. (In what follows, we use primes for some of our constants to distinguish them from their corresponding constants in Proposition 3.2.)

Lemma 7.2. For all $\alpha, \delta_{B}^{\prime}>0$ there exists $\delta_{A}^{\prime}>0$ such that for all integers $\ell$ and $r_{B}^{\prime}$, there exist $\varepsilon^{\prime}>0$ and integer $r_{A}^{\prime}$ so that whenever $\mathcal{H}$ and $P$ satisfy the hypothesis of Setup 2.1 with constants $\alpha, \ell$ and $\varepsilon^{\prime}$ and with $n$ sufficiently large, the following holds:

Suppose there exist $\delta_{B}^{\prime} n^{2}$ pairs $\{x, y\} \in\left(\begin{array}{c}V_{1} \\ 2\end{array}\right)$ for which $L_{x y}$ is not $\left(\alpha^{2} / \ell, \delta_{B}^{\prime}, r_{B}^{\prime}\right)$-regular and for which witnesses $U_{1}^{x y}, \ldots, U_{r_{B}^{\prime}}^{x y} \subseteq N_{P^{12}}(x, y)$ and $W_{1}^{x y}, \ldots, W_{r_{B}^{\prime}}^{x y} \subseteq N_{P^{13}}(x, y)$ against the $\left(\alpha^{2} / \ell, \delta_{B}^{\prime}, r_{B}^{\prime}\right)$-regularity of $L_{x y}$ are given. Then $\mathcal{H}$ is not $\left(\delta_{A}^{\prime}, r_{A}^{\prime}\right)$-regular w.r.t. $P$, and there exists an algorithm $\mathbf{A}_{7.2}$ which, in time $O\left(n^{5}\right)$, converts the witnesses $U_{1}^{x y}, \ldots, U_{r_{B}^{\prime}}^{x y}$ and $W_{1}^{x y}, \ldots, W_{r_{B}^{\prime}}^{x y}$, over the $\delta_{B}^{\prime} n^{2}$ pairs $\{x, y\}$ above, into a witness $\mathbf{Q}_{r_{A}^{\prime}}=\left(Q_{1}, \ldots, Q_{r_{A}^{\prime}}\right)$ of the $\left(\delta_{A}^{\prime}, r_{A}^{\prime}\right)$-irregularity of $\mathcal{H}$ w.r.t. $P$.

To prove that Statement (2) of Proposition 3.2 follows from Lemma 7.2, we shall need the following auxiliary fact. (This fact will allow us to build, in the context of Lemma 7.2, the witnesses $U_{1}^{x y}, \ldots, U_{r_{B}^{\prime}}^{x y}$ and $W_{1}^{x y}, \ldots, W_{r_{B}^{\prime}}^{x y}$.)

Fact 7.3. For all $\beta, \delta_{2}>0$, there exists $\delta_{1}>0$ so that for all $d>0$, there exist integer $R$ and $\zeta>0$ so that the following holds: let $F \subseteq G$ be bipartite graphs with bipartition $U \cup W$, where $|U|,|W|$ are sufficiently large, and $G$ is $(d, \zeta)$-regular. If there exist $\delta_{2}|U|^{2}$ pairs $\left\{u, u^{\prime}\right\} \in$ $\left(\begin{array}{l}U \\ 2\end{array}\right)$ satisfying

$$
\operatorname{deg}_{F}\left(u, u^{\prime}\right) \neq(\beta d)^{2}|W|\left(1 \pm \delta_{2}\right)
$$

then $F$ is not $\left(\beta d, \delta_{1}, R\right)$-regular, and there exists an algorithm $\mathbf{A}_{7.3}$ which, in time $O\left(|U|^{2}|W|\right)$ constructs witnesses $U_{1}, \ldots, U_{R} \subseteq U$ and $W_{1}, \ldots, W_{R} \subseteq W$ of the $\left(\beta d, \delta_{1}, R\right)$-irregularity of $F$.

\footnotetext{
${ }^{2}$ In [11], a slightly stronger statement is proven which includes an additional parameter $\beta$. To derive Lemma 7.2 from the original Lemma 5.8 of [11], one sets $\beta=\delta_{B}^{\prime} / 2$ and then uses that we may take $\varepsilon<\delta_{B}^{\prime} / 2$. (For the definition of $V_{1}^{\text {good }}$ in Lemma 5.8 of [11], see Definition 4.4 (p. 303).)
} 
Fact 7.3 was proved, without any focus to the algorithmic assertion, as Fact 8.12, p. 395, of [35]. For completeness, we repeat the proof at the end of the paper and emphasize the algorithmic aspects.

7.2. Proof of Proposition 3.2. We begin our proof by formally describing the constants involved. The Reader not interested in the determination of these constants may skip ahead.

7.2.1. Constants. As in the quantification of Proposition 3.2, let $\alpha, \delta_{B}>0$ be given. In Fact 7.3, put $\beta=\alpha^{2}$ and

$$
\delta_{2}=\frac{\delta_{B}^{3}}{1000} \leq \frac{\delta_{B}^{2}}{4}
$$

Let

$$
\delta_{1}=\delta_{1}^{(7.3)}\left(\alpha^{2}, \delta_{2}\right) \leq \frac{\delta_{B}^{2}}{2}
$$

be the constant guaranteed by Fact 7.3 (where we may assume, w.l.o.g., that $\delta_{1} \leq \delta_{B}^{2} / 2$ ). Putting $\delta_{B}^{\prime}=\delta_{1}$, let

$$
\delta_{A}^{\prime}=\delta_{A,(7.2)}^{\prime}\left(\alpha, \delta_{B}^{\prime}\right)
$$

be the constant guaranteed by Lemma 7.2. For Proposition 3.2, we take the promised constant $\delta_{A}$ as

$$
\delta_{A}=\delta_{A}^{\prime}=\delta_{A,(7.2)}^{\prime}\left(\alpha, \delta_{B}^{\prime}\right) .
$$

Now, as in Proposition 3.2, let integer $\ell$ be given. In Fact 7.3, set $d=1 / \ell$ and let

$$
\zeta=\zeta^{(7.3)}\left(\alpha^{2}, \delta_{2}, \delta_{1}, 1 / \ell\right) \text { and } R=R^{(7.3)}\left(\alpha^{2}, \delta_{2}, \delta_{1}, 1 / \ell\right)
$$

be the constants guaranteed by Fact 7.3. In Lemma 7.2, set $r_{B}^{\prime}=R$ and let

$$
\varepsilon_{A,(7.2)}^{\prime}=\varepsilon_{A,(7.2)}^{\prime}\left(\alpha, \delta_{B}^{\prime}, \delta_{A}^{\prime}, \ell, r_{B}^{\prime}\right) \quad r_{A,(7.2)}^{\prime}=r_{A,(7.2)}^{\prime}\left(\alpha, \delta_{B}^{\prime}, \delta_{A}^{\prime}, \ell, r_{B}^{\prime}\right)
$$

be the constants guaranteed by Lemma 7.2. Let

$$
\varepsilon^{(4.6)}=\varepsilon^{(4.6)}\left(\alpha, \delta_{B}, \ell\right)
$$

be the constant guaranteed by Proposition 4.6. For Proposition 3.2, we take

$$
r=r_{A,(7.2)}^{\prime} \quad \text { and } \quad \varepsilon=\min \left\{\varepsilon_{A,(7.2)}^{\prime}, \zeta^{2}, \varepsilon^{(4.6)}\right\} .
$$

This concludes our definitions of the constants.

7.2.2. The algorithm. We now prove Statement (2) of Proposition 3.2. Let $\mathcal{H}$ and $P=$ $P^{12} \cup P^{23} \cup P^{13}$ be given as in Setup 2.1 with constants $\alpha, \delta_{B}, \delta_{A}, \ell, r, \varepsilon$ described in (111)(117). Suppose $\mathcal{H}$ is $\left(\alpha, \delta_{B}\right)$-excessive w.r.t. $P$. We establish an algorithm $\mathbf{A}_{3.2}$ which, in time $O\left(n^{5}\right)$, constructs a witness $\mathbf{Q}_{r}=\left(Q_{1}, \ldots, Q_{r}\right)$ of the $\left(\delta_{A}, r\right)$-irregularity of $\mathcal{H}$ w.r.t. $P$. In the immediate sequel, we list the steps of the algorithm $\mathbf{A}_{3.2}$. Afterwards, we fill in all details.

\footnotetext{
Algorithm $\mathbf{A}_{3.2}$

Given. $\mathcal{H}$ and $P$, as in the hypothesis of Proposition 3.2, where $\mathcal{H}$ is $\left(\alpha, \delta_{B}\right)$-excessive w.r.t. $P$

Output. In time $O\left(n^{5}\right)$, a witness $\mathbf{Q}_{r}=\left(Q_{1}, \ldots, Q_{r}\right)$ of the $\left(\delta_{A}, r\right)$-irregularity of $\mathcal{H}$ w.r.t. P.
}

\section{Steps.}


(1) Statement (2)-(b) of Proposition 4.6 guarantees $\delta_{2} n^{2}$ pairs $\{x, y\} \in\left(\begin{array}{c}V_{1} \\ 2\end{array}\right)$ so that for each such $\{x, y\}$, there are $\delta_{2}\left[\operatorname{deg}_{P^{12}}(x, y)\right]^{2}$ pairs $\{a, b\} \in\left(\begin{array}{c}N_{P^{12}}(x, y) \\ 2\end{array}\right)$, each of which satisfies

$$
\operatorname{deg}_{L_{x y}}(a, b) \neq\left(\frac{\alpha^{2}}{\ell}\right)^{2} \operatorname{deg}_{P^{13}}(x, y)\left(1 \pm \delta_{2}\right) .
$$

The pairs $\{x, y\}$ and corresponding pairs $\{a, b\}$ can be found in time $O\left(n^{5}\right)$.

(2) For a fixed $\{x, y\}$ from Step 1, Algorithm $\mathbf{A}_{7.3}$ (cf. Fact 7.3) constructs, in time $O\left(n^{3}\right)$, witnesses $U_{1}^{x y}, \ldots, U_{R}^{x y}$ and $W_{1}^{x y}, \ldots, W_{R}^{x y}$ of the $\left(\alpha^{2} / \ell, \delta_{1}, R\right)$-irregularity of $L_{x y}$. Over all $\{x, y\}$ from Step 1, we build a family of witnesses in time $O\left(n^{5}\right)$.

(3) Algorithm $\mathbf{A}_{7.2}$ (cf. Lemma 7.2) converts, in time $O\left(n^{5}\right)$, the witnesses $U_{1}^{x y}, \ldots, U_{R}^{x y}$ and $W_{1}^{x y}, \ldots, W_{R}^{x y}$, over all $\{x, y\}$ from Step 1, into a witness $\mathbf{Q}_{r}=\left(Q_{1}, \ldots, Q_{r}\right)$ of the $\left(\delta_{A}, r\right)$-irregularity of $\mathcal{H}$ w.r.t. $P$.

We now establish the steps above, beginning with the first.

Step 1. We appeal to Statement (2)-(b) of Proposition 4.6, setting, in that context, $\delta=\delta_{B}$. Since $\mathcal{H}$ is $\left(\alpha, \delta_{B}\right)$-excessive w.r.t. $P$, Statement (2)-(b) of Proposition 4.6 guarantees at least (with $\varepsilon \ll 1 / \ell$ )

$$
\frac{\delta_{B}^{3}}{27}\left|\mathcal{K}_{2,2}^{(2)}\left(P^{12}\right)\right| \stackrel{(43)}{\geq} \frac{\delta_{B}^{3}}{28 \ell^{4}}\left(\begin{array}{c}
n \\
2
\end{array}\right)^{2} \geq \frac{\delta_{B}^{3}}{115 \ell^{4}} n^{4}
$$

elements $(\{x, y\},\{a, b\}) \in \mathcal{K}_{2,2}^{(2)}\left(P^{12}\right)$ for which

$$
\operatorname{deg}_{L_{x y}}(a, b) \neq\left(\frac{\alpha^{2}}{\ell}\right)^{2}\left|N_{P^{13}}(x, y)\right|\left(1 \pm 2 \frac{\delta_{B}^{3}}{27}\right) .
$$

Our choice of $\delta_{2}<2 \delta_{B}^{3} /(27)$ in (111) then ensures that these elements also satisfy

$$
\operatorname{deg}_{L_{x y}}(a, b) \neq\left(\frac{\alpha^{2}}{\ell}\right)^{2}\left|N_{P^{13}}(x, y)\right|\left(1 \pm \delta_{2}\right) .
$$

These elements can be found in time $O\left(n^{5}\right)$. In the next two steps, we shall consider only those elements $(\{x, y\},\{a, b\})$ for which

$$
\frac{n}{2 \ell^{2}} \leq \operatorname{deg}_{P^{12}}(x, y), \operatorname{deg}_{P^{13}}(x, y) \leq 2 \frac{n}{\ell^{2}},
$$

of which the $\left(\ell^{-1}, \varepsilon\right)$-regularity of $P^{12}$ and $P^{13}$ implies there must be at least (using $\varepsilon \ll 1 / \ell$ )

$$
\frac{\delta_{B}^{3}}{115 \ell^{4}} n^{4}-4 \varepsilon n^{4}>\frac{\delta_{B}^{3}}{120 \ell^{4}} n^{4}
$$

many. Using (119) and (120), a simple pigeon-hole calculation then shows that there must be at least

$$
\frac{\delta_{B}^{3}}{500} n^{2} \stackrel{(111)}{\geq} \delta_{2} n^{2}
$$

pairs $\{x, y\} \in\left(\begin{array}{c}V_{1} \\ 2\end{array}\right)$, each of which has at least

$$
\frac{\delta_{B}^{3}}{250} \frac{n^{2}}{\ell^{4}} \geq \frac{\delta_{B}^{3}}{1000}\left[\operatorname{deg}_{P^{12}}(x, y)\right]^{2} \stackrel{(111)}{=} \delta_{2}\left[\operatorname{deg}_{P^{12}}(x, y)\right]^{2}
$$


pairs $\{a, b\} \in\left(\begin{array}{c}N_{P^{12}}(x, y) \\ 2\end{array}\right)$ for which (118) holds. Let $\left(\begin{array}{c}V_{1} \\ 2\end{array}\right)_{\text {bad }}$ denote the set of these pairs $\{x, y\}$, and for each such $\{x, y\}$, let $\left(\begin{array}{c}N_{P} 12 \\ 2\end{array}(x, y)\right.$ bad denote its set of corresponding pairs $\{a, b\}$.

Step 2. Fix a pair $\{x, y\} \in\left(\begin{array}{c}V_{1} \\ 2\end{array}\right)_{\text {bad }}$. As in Fact 7.3, set $\beta=\alpha^{2}, d=\ell^{-1}$ and $\zeta=\varepsilon^{1 / 2}$ and set

$$
F=L_{x y}, \quad G=P_{x y}, \quad U=N_{P^{12}}(x, y), \quad W=N_{P^{13}}(x, y) .
$$

Since the graph $P_{x y}$ is $\left(1 / \ell, \varepsilon^{1 / 2}\right.$ )-regular (cf. (41)) where $\varepsilon \leq \zeta^{2}$ (see (117)), the graph $G$ is $(d, \zeta)$-regular. The set $\left(\begin{array}{c}N_{P^{12}}(x, y) \\ 2\end{array}\right)_{\text {bad }}=\left(\begin{array}{c}U \\ 2\end{array}\right)_{\text {bad }}$ corresponds to a collection of $\delta_{2}|U|^{2}$ pairs $\left\{u, u^{\prime}\right\} \in\left(\begin{array}{c}U \\ 2\end{array}\right)$ for which

$$
\operatorname{deg}_{F}\left(u, u^{\prime}\right)=\operatorname{deg}_{L_{x y}}\left(u, u^{\prime}\right) \neq\left(\frac{\alpha^{2}}{\ell}\right)\left|N_{P^{13}}(x, y)\right|\left(1 \pm \delta_{2}\right)=(\beta d)^{2}|W|\left(1 \pm \delta_{2}\right) .
$$

By our choice of $\delta_{1}$ in (112), $R$ in (115) and $\zeta$ in (115), Fact 7.3 applies to say that the graph $F=L_{x y}$ is $\left(\alpha^{2} / \ell, \delta_{1}, R\right)$-irregular. Moreover, Algorithm $\mathbf{A}_{7.3}$ constructs, in time $O\left(|U|^{2}|W|\right)=$ $O\left(n^{3}\right)$, witnesses $U_{1}^{x y}, \ldots, U_{R}^{x y} \subseteq U$ and $W_{1}^{x y}, \ldots, W_{R}^{x y} \subseteq W$ of the $\left(\alpha^{2} / \ell, \delta_{1}, R\right)$-irregularity of $L_{x y}$. In time $O\left(n^{5}\right)$, we repeat this process over all $\{x, y\} \in\left(\begin{array}{c}V_{1} \\ 2\end{array}\right)$.

Step 3. We apply Lemma 7.2 to $\mathcal{H}, P$ and the collection of witnesses $U_{1}^{x y}, \ldots, U_{R}^{x y}$ and $W_{1}^{x y}, \ldots, W_{R}^{x y}$ over $\{x, y\} \in\left(\begin{array}{c}V_{1} \\ 2\end{array}\right)_{\text {bad }}$. To begin, we recall $\mathcal{H}$ and $P$ are as in Setup 2.1 with constants $\alpha, \ell$ and $\varepsilon$. Step 2 constructed witnesses $U_{1}^{x y}, \ldots, U_{R}^{x y}$ and $W_{1}^{x y}, \ldots, W_{R}^{x y}$ of the $\left(\alpha^{2} / \ell, \delta_{1}, R\right)$-irregularity of $L_{x y}$ for $\delta_{1} n^{2}$ pairs $\{x, y\} \in\left(\begin{array}{c}V_{1} \\ 2\end{array}\right)_{\text {bad }}$. By our choice of $\delta_{A}=\delta_{A}^{\prime}$ in (113) and (114) and $r_{A}=r_{A,(7.2)}^{\prime}$ and $\varepsilon \leq \varepsilon_{A,(7.2)}^{\prime}$ in (116) and (117), Lemma 7.2 applies to say that $\mathcal{H}$ is $\left(\delta_{A}, r\right)$-irregular w.r.t. $P$. Moreover, Algorithm $\mathbf{A}_{7.2}$ converts the witnesses $U_{1}^{x y}, \ldots, U_{R}^{x y}$ and $W_{1}^{x y}, \ldots, W_{R}^{x y}$, over all $\{x, y\} \in\left(\begin{array}{c}V_{1} \\ 2\end{array}\right)_{\text {bad }}$, into a witness $\mathbf{Q}_{r}=\left(Q_{1}, \ldots, Q_{r}\right)$ of the $\left(\delta_{A}, r\right)$-irregularity of $\mathcal{H}$ w.r.t. $P$.

7.3. Proof of Fact 7.3. For simplicity, we shall give an informal description of the constants. Let $\beta, \delta_{2}>0$ be given. Without loss of generality, we shall assume $\delta_{2} \ll \beta$. Choose $0<\delta_{1} \ll \delta_{2}$. Let $d>0$ be given. Set $R=\delta_{2}^{3} / d$ and choose $0<\zeta \ll \min \left\{d, \delta_{1}\right\}$. With these constants, let $F$ and $G$ be given as in the hypothesis of Fact 7.3. We show that in time $O\left(|U|^{2}|W|\right)$, we may construct witnesses $U_{1}, \ldots, U_{R} \subseteq U$ and $W_{1}, \ldots, W_{R} \subseteq W$ against the $\left(\beta d, \delta_{1}, R\right)$-regularity of $F$.

Denote by $U^{-}$the set of vertices $u \in U$ for which $\operatorname{deg}_{F}(u)<\beta d|W|\left(1-\delta_{1}\right)$. If $\left|U^{-}\right|>\delta_{1}|U|$, then we are done. Indeed, we may take $U_{1}=U^{-}$and $W_{1}=W$ so that the 1 -tuple $U_{1}, W_{1}$ satisfies $\left|U_{1}\right|\left|W_{1}\right|>\delta_{1}|U||W|$ but, by construction, $\left|F \cap\left(U_{1} \times W_{1}\right)\right|<\beta d\left|U_{1}\right|\left|W_{1}\right|\left(1-\delta_{1}\right)$. As such, $F$ is not $\left(\beta d, \delta_{1}, 1\right)$-regular, and therefore, not $\left(\beta d, \delta_{1}, R\right)$-regular, and the witness $U_{1}$, $W_{1}$ is found in time $O(|U||W|)$. In the remainder of the proof, we shall therefore assume $\left|U^{-}\right| \leq \delta_{1}|U|$.

Now, suppose that there exist $\delta_{2}|U|^{2}$ pairs $\left\{u, u^{\prime}\right\} \in\left(\begin{array}{c}U \\ 2\end{array}\right)$ for which

$$
\operatorname{deg}_{F}\left(u, u^{\prime}\right) \neq(\beta d)^{2}|W|\left(1 \pm \delta_{2}\right) .
$$

Then, at least $\left(\delta_{2} / 2\right)|U|^{2}$ pairs must have codegree in $F$ which is, say, too small, and these pairs can be found in time $O\left(|U|^{2}|V|\right)$. We can then find a set $U_{0} \subseteq U,\left|U_{0}\right| \geq\left(\delta_{2} / 4\right)|U|$, so that for each $u \in U_{0}$, there exists a set $U_{u} \subseteq U,\left|U_{u}\right| \geq\left(\delta_{2} / 4\right)|U|$, so that for each $u^{\prime} \in U_{u}$,

$$
\operatorname{deg}_{F}\left(u, u^{\prime}\right)<(\beta d)^{2}|W|\left(1-\delta_{2}\right) .
$$


Moreover, the set $U_{0}$ and corresponding sets $U_{u}, u \in U_{0}$, can be found in time $O\left(|U|^{2}\right)$. We show that the graph $F$ cannot be $\left(\beta d, \delta_{1}, R\right)$-regular, and we shall construct a witness $U_{1}, \ldots, U_{R}$, $W_{1}, \ldots, W_{R}$ to this effect.

To this end, we find in time $O\left(|U|^{2}|W|\right)$ a subset $U_{0}^{*}=\left\{u_{1}, \ldots, u_{R}\right\} \subset U_{0} \backslash U^{-}$with the property that for each $1 \leq i<j \leq R$,

$$
\operatorname{deg}_{G}\left(u_{i}, u_{j}\right) \leq 2 d^{2}|W|
$$

Indeed, it is well known from the $(d, \zeta)$-regularity of $G$ that all but $4 \zeta|U|^{2}$ pairs $u_{i}, u_{j}$ satisfy (121). Let $\Gamma$ be the graph of the pairs $u_{i}, u_{j}$ not satisfying (121). Pick $u_{1} \in U_{0} \backslash U^{-}$to be any vertex with $\operatorname{deg}_{\Gamma}\left(u_{1}\right) \leq 3 \zeta^{1 / 2}|U|$. There are at least

$$
\left|U_{0}\right|-\delta_{1}|U|-3 \zeta^{1 / 2}|U| \geq\left(\frac{\delta_{2}}{4}-\delta_{1}-3 \zeta^{1 / 2}\right)|U|>0
$$

choices for $u_{1}$. If $\left\{u_{1}, \ldots, u_{t-1}\right\} \subset U_{0} \backslash U^{-}$have already been chosen, $t-1<R$, pick any $u_{t} \in U_{0} \backslash\left(U^{-} \cup \bigcup_{1 \leq i \leq t-1} N_{\Gamma}\left(u_{i}\right)\right)$ with $\operatorname{deg}_{\Gamma}\left(u_{t}\right) \leq 3 \zeta^{1 / 2}|U|$. There are at least

$\left|U_{0}\right|-\delta_{1}|U|-3(t-1) \zeta^{1 / 2}|U|-3 \zeta^{1 / 2}|U| \geq\left(\frac{\delta_{2}}{4}-\delta_{1}-3 R \zeta^{1 / 2}\right)|U|=\left(\frac{\delta_{2}}{4}-\delta_{1}-\frac{3 \zeta^{1 / 2}}{d}\right)|U|>0$ choices for $u_{t}$.

We now give the promised witness. For each $1 \leq i \leq R$, let $U_{i}=U_{u_{i}}$, where $u_{i} \in U_{0}^{*}$, and let $W_{i}=N_{F}\left(u_{i}\right)$. This $R$-tuple was constructed in time $O\left(|U|^{2}|W|\right)$. We now verify that the $R$ tuple is a witness for the $\left(\beta d, \delta_{1}, R\right)$-irregularity of $F$. To that end, we see by inclusion-exclusion that

$$
\left|\bigcup_{i=1}^{R}\left(U_{i} \times W_{i}\right)\right| \geq \sum_{i=1}^{R}\left|U_{i} \times W_{i}\right|-\sum_{1 \leq i<j \leq R}\left|\left(U_{i} \times W_{i}\right) \cap\left(U_{j} \times W_{j}\right)\right| .
$$

For a fixed $1 \leq i \leq R$, note that

$$
\left|U_{i} \times W_{i}\right|=\left|U_{i}\right| \operatorname{deg}_{F}\left(u_{i}\right) \geq\left|U_{i}\right|(\beta d)|W|\left(1-\delta_{1}\right) \geq \frac{\delta_{2} \beta d}{4}|U||W|\left(1-\delta_{1}\right) \geq \frac{\delta_{2} \beta d}{8}|U||W|
$$

and for a fixed $1 \leq i<j \leq R$,

$$
\left|\left(U_{i} \times W_{i}\right) \cap\left(U_{j} \times W_{j}\right)\right| \leq|U| \operatorname{deg}_{G}\left(u_{i}, u_{j}\right) \leq 2 d^{2}|U||W| .
$$

As such, with $R=\delta_{2}^{3} / d$ and $\delta_{1} \ll \delta_{2} \ll \beta$, we have

$$
\left|\bigcup_{i=1}^{R}\left(U_{i} \times W_{i}\right)\right| \geq \frac{\delta_{2}^{4} \beta}{8}|U||W|-\delta_{2}^{6}|U||W|>\frac{\delta_{2}^{4} \beta}{16}|U||W|>\delta_{1}|U||W| .
$$

To finish verifying the witness, note that implicit in the work above is the inequality

$$
\left|\bigcup_{i=1}^{R}\left(U_{i} \times W_{i}\right)\right| \geq \beta d|W|\left(1-\delta_{1}\right) \sum_{i=1}^{R}\left|U_{i}\right|-\delta_{2}^{6}|U||W| .
$$


We have, with $\delta_{1} \ll \delta_{2} \ll \beta$,

$$
\begin{aligned}
& \left|F \cap \bigcup_{i=1}^{R}\left(U_{i} \times W_{i}\right)\right| \leq \sum_{i=1}^{R}\left|F \cap\left(U_{i} \times W_{i}\right)\right|=\sum_{i=1}^{R} \sum_{u^{\prime} \in U_{i}} \operatorname{deg}_{F}\left(u_{i}, u^{\prime}\right) \\
& <\sum_{i=1}^{R} \sum_{u^{\prime} \in U_{i}}(\beta d)^{2}|W|\left(1-\delta_{2}\right)=(\beta d)^{2}|W|\left(1-\delta_{2}\right) \sum_{i=1}^{R}\left|U_{i}\right| \\
& \stackrel{(123)}{\leq}(\beta d)^{2}|W|\left(1-\delta_{2}\right) \frac{\left[\left|\bigcup_{i=1}^{R}\left(U_{i} \times W_{i}\right)\right|+\delta_{2}^{6}|U||W|\right]}{\beta d|W|\left(1-\delta_{1}\right)} \\
& =\beta d\left(\frac{1-\delta_{2}}{1-\delta_{1}}\right)\left[1+\frac{\delta_{2}^{6}|U||W|}{\left|\bigcup_{i=1}^{R}\left(U_{i} \times W_{i}\right)\right|}\right]\left|\bigcup_{i=1}^{R}\left(U_{i} \times W_{i}\right)\right| \\
& \stackrel{(122)}{\leq} \beta d\left(\frac{1-\delta_{2}}{1-\delta_{1}}\right)\left(1+\frac{16 \delta_{2}^{2}}{\beta}\right)\left|\bigcup_{i=1}^{R}\left(U_{i} \times W_{i}\right)\right| \\
& <\beta d\left(1-\frac{\delta_{2}}{2}\right)\left|\bigcup_{i=1}^{R}\left(U_{i} \times W_{i}\right)\right|<\beta d\left(1-\delta_{1}\right)\left|\bigcup_{i=1}^{R}\left(U_{i} \times W_{i}\right)\right| .
\end{aligned}
$$

This completes the proof.

\section{REFERENCES}

[1] Alon, N., Duke, R., Lefmann, H., Rödl, V., Yuster, R., "The algorithmic aspects of the Regularity Lemma", Journal of Algorithms Vol. 16, pp. 80-109, 1994

[2] Alon, N., Duke, R., Lefmann, H., Rödl, V., Yuster, R., "The algorithmic aspects of the Regularity Lemma (extended abstract)", Proceedings of the IEEE Symposium on Foundations of Computer Science, pp. 473481, 1992

[3] Alon, N., Fischer, E., Krivelevich, M., Szegedy, M., "Efficient testing of large graphs", Combinatorica 20, pp 451-476, 2002.

[4] Alon, N., Shapira, A., Sudakov, B., "Additive approximation for edge-deletion problems", Proceedings of the IEEE Symposium on Foundations of Computer Science, pp. 419-428, 2005

[5] Chung, F.R.K., "Regularity lemmas for hypergraphs and quasi-randomness", Random Structures and Algorithms Vol. 2, pp. 241-252, 1991

[6] Chung, F.R.K., "Regularity lemmas for hypergraphs and quasi-randomness", Random Structures and Algorithms Vol. 2, pp. 241-252, 1991

[7] Chung, F.R.K., Graham, R.L., "Quasi-random set systems" J. Amer. Math. Soc. 4 (1991), pp. 151-196.

[8] Chung, F.R.K., Graham, R.L., Wilson, R.M., Quasi-random graphs, Combinatorica 9 no. 4, 345-362, 1989.

[9] Czygrinow, A., Nagle, B., "On small subsystems of uniformly distributed 3-graphs", submitted.

[10] Czygrinow, A., Rödl, V., "An algorithmic regularity lemma for hypergraphs", SIAM Journal on Computing, 30, pp 1041-1066, 2000.

[11] Dementieva, Y., Haxell, P., Nagle, B., Rödl, V., "On characterizing hypergraph regularity", Random Structures \& Algorithms, Vol 21, 3-4, pp 293-395, 2002.

[12] Dor, D., Tarsi, M., "Graph decomposition is NPC - a complete proof of Holyer's conjecture", Proceedings of the ACM Symposium on Theory of Computing, pp. 252-263, 1992.

[13] Duke, R., Lefmann, H., Rödl, V., "A fast approximation algorithm for computing the frequencies of subgraphs of a given graph", SIAM Journal of Computing Vol. 24, pp. 598-620, 1995

[14] Frankl, P., Rödl, V., "Extremal problems on set systems", Random Structures and Algorithms, 20 (2002), no. 2, pp 131-164.

[15] Frankl, P., Rödl, V., "The uniformity lemma for hypergraphs", Graphs and Combinatorics Vol. 8, no.4, pp. 309-312, 1992 
[16] Frieze, A., Kannan, R., "The Regularity Lemma and approximation schemes for dense problems", Proceedings of the IEEE Symposium on Foundations of Computer Science, pp. 12-20, 1996

[17] Frieze, A., Kannan, R., "Quick approximation to matrices and applications", Combinatorica Vol. 19, pp. $175-220,1999$

[18] Gowers, W.T., "Quasirandomness, counting and regularity for 3-uniform hypergraphs", Combinatorics, Probability and Computing (2006) 15, 143-184.

[19] Gowers, W.T., "Hypergraph regularity and the multidimensional Szemerédi Theorem", preprint.

[20] Haxell, P., Nagle, B., Rödl, V., "An algorithmic version of the hypergraph regularity method (extended abstract)", Proceedings of the IEEE Symposium on Foundations of Computer Science, pp. 439-446, 2005.

[21] Haxell, P., Nagle, B., Rödl, V., "Integer and fractional packings in dense 3-uniform hypergraphs", Random Structures \& Algorithms, Vol 22, 3, 2003, pp 248-310.

[22] Haxell, P., Rödl, V., "Integer and fractional packings in dense graphs", Combinatorica, 21 (2001), no. 1, pp. 13 - 38.

[23] Kohayakawa, Y., Nagle, B., Rödl, V., "Hereditary properties of triple systems", Combinatorics, Probability and Computing, 12, 2003, pp 155-189.

[24] Kohayakawa, Y., Nagle, B., Rodl, V., "Efficient testing of hypergraphs", ICALP 2002, 29th International Colloquium on Automata, Languages and Programming, (Malaga, Spain), July 2002, Lecture Notes in Computer Science 2286, Springer, Berlin, 2002, pp 278-292.

[25] Kohayakawa, Y., Rödl, V., Skokan, J., "Equivalent Conditions of Hypergraph Regularity", Journal of Combinatorial Theory, Series A, 97, 2002, no. 2, pp. 307-352.

[26] Kohayakawa, Y., Rödl, V., Thoma, L., "An optimal algorithm for checking regularity (extended abstract), The $13^{\text {th }}$ Annual ACM-SIAM Symposium on Discrete Algorithms (SODA 2002), San Francisco, CA, 6 to 8 January 2002, pp. 277-286.

[27] Kohayakawa, Y., Rödl, V., Thoma, L., "An optimal algorithm for checking regularity", SIAM Journal on Computing, 32, pp. 1210-1235, 2003.

[28] J. Komlós, A. Shokoufandeh, M. Simonovits, and E. Szemerédi, "The regularity lemma and its applications in graph theory, Theoretical aspects of computer science (Tehran, 2000), Lecture Notes in Comput. Sci., vol. 2292, Springer-Berlin, 2002, pp. 84-112.

[29] J. Komlós, M. Simonovits, "Szemerédi's Regularity Lemma and its applications in graph theory", Combinatorics, Paul Erdös is eighty, Vol. 2, pp. 295-352, 1996.

[30] Nagle, B., Rödl, V., "Regularity properties for triple systems", Random Structures \& Algorithms, Vol. 23, 3, 2003, pp. 264-332.

[31] Nagle, B., Rödl, V., "The asymptotic number of triple systems not containing a fixed one", Discrete Mathematics, Vol 235, 2001, pp. 271-290.

[32] Nagle, B., Rödl, V., Schacht, M., "The counting lemma for regular k-uniform hypergraphs", Random Structures \& Algorithms, Vol 26, Issue 2, 2006,, pp. 1-67.

[33] Nešetřil, J., Rödl, V., "Partitions of finite relational and set systems", Journal of Combinatorial Theory, Ser. A, Vol 22, 1977, pp. 289-312.

[34] Nešetřil, J., Rödl, V., "Two proofs of the Ramsey property of the class of finite hypergraphs", European Journal of Combinatorics, Vol 3, 1982, pp. 347-352.

[35] Peng, Y., Rödl, V., Skokan, J., "Counting small subgraphs in a 3-hypergraph", Combinatorics, Probability and Computing (2005) 14, 371-413.

[36] Rödl, V., Ruciński, A., "Ramsey properties of random hypergraphs", J. Combin. Theory Ser. A, Vol. 81, no. 1, pp. 1-33, 1998

[37] Rödl, V., Ruciński, A., Szemerédi, E., "A Dirac-type theorem for 3-uniform hypergraphs", Combinatorics, Probability and Computing 15 (2006), no. 1-2, pp. 229-251.

[38] Rödl, V., Schacht, M., Siggers, M., Tokushige, N., "Fractional packings of hypergraphs", Journal of Combinatorial Theory, Ser. B, to appear.

[39] Rödl, V., Schacht, M., Tengan, E., Tokushige, N., "Density theorems and the Hypergraph Regularity Method", Israel Journal of Mathematics 152, pp. 371-380.

[40] Rödl, V., Skokan, J., "Regularity lemma for k-uniform hypergraphs", Random Structures \& Algorithms, 25, pp. 1-42, 2004.

[41] Rödl, V., Skokan, J., "Applications of the regularity lemma for k-uniform hypergraphs", Random Structures and Algorithms, Vol 26, Issue 2, 2006, pp. 180-194. 
[42] Skokan, J., Thoma, L., "Bipartite subgraphs and quasi-randomness", Graphs and Combinatorics, 20 (2), 2004, pp. 255-262.

[43] Solymosi, J., "A note on a question of Erdos and Graham", Combinatorics, Probability and Computing, 2004, Vol 13, Issue 2, 263-267.

[44] Szemerédi, E., "On sets of integers containing no $k$ elements in arithmetic progression", Acta Arithmetica 27, pp. 199-245, 1975

[45] Szemerédi, E., "Regular partitions of graphs", in Problèmes en Combinatoire et Théorie des Graphes, Proc. Colloque Inter. CNRS, (Bermond, J.-C., Fournier, J.-C., Las Vergnas, M., Sotteau, D., eds), pp. 399-401, 1978

[46] Tao, T., "A variant of the hypergraph removal lemma", Journal of Combinatorial Theory, Ser. A 113 (2006), pp. 1257-1280.

[47] Tao, T., "The Gaussian primes contain arbitrarily shaped constellations", J. d'Analyse Mathematique, to appear.

[48] Thomason, A., "Dense expanders and pseudo-random bipartite graphs", Graph Theory and Combinatorics (Cambridge, 1988) Discrete Math. Vol. 75, no.1-3, pp. 381-386, 1989

Department of Combinatorics and Optimization, University of Waterloo, Waterloo, Ont., Canada, N2L 3G1.

E-mail address: pehaxell@math.uwaterloo.ca.

Department of Mathematics and Statistics, University of South Florida, Tampa, FL 33620, USA.

E-mail address: bnagle@math.usf.edu.

Department of Mathematics and Computer Science, Emory University, Atlanta, Ga 30322, USA.

E-mail address: rodl@mathcs.emory.edu. 